\title{
The search for high entropy alloys: a high-throughput ab-initio approach
}

\author{
Yoav Lederer, ${ }^{1,2}$ Cormac Toher, ${ }^{1}$ Kenneth S. Vecchio, ${ }^{3}$ and Stefano Curtarolo ${ }^{4,5, *}$ \\ ${ }^{1}$ Department of Mechanical Engineering and Materials Science, \\ Duke University, Durham, North Carolina 27708, USA \\ ${ }^{2}$ Department of Physics, NRCN, Beer-Sheva, 84190, Israel \\ ${ }^{3}$ Department of NanoEngineering, University of California San Diego, La Jolla, CA 92093 \\ ${ }^{4}$ Materials Science, Electrical Engineering, Physics and Chemistry, Duke University, Durham NC, 27708 \\ ${ }^{5}$ Fritz-Haber-Institut der Max-Planck-Gesellschaft, 14195 Berlin-Dahlem, Germany
}

(Dated: July 16, 2018)

While the ongoing search to discover new high-entropy systems is slowly expanding beyond metals, a rational and effective method for predicting "in silico" the solid solution forming ability of multi-component systems remains yet to be developed. In this article, we propose a novel high-throughput approach, called "LTVC", for estimating the transition temperature of a solid solution: ab-initio energies are incorporated into a mean field statistical mechanical model where an order parameter follows the evolution of disorder. The LTVC method is corroborated by Monte Carlo simulations and the results from the current most reliable data for binary, ternary, quaternary and quinary systems $(96.6 \% ; 90.7 \%$; $100 \%$ and $100 \%$, of correct solid solution predictions, respectively). By scanning through the many thousands of systems available in the AFLOW consortium repository, it is possible to predict a plethora of previously unknown potential quaternary and quinary solid solutions for future experimental validation.

\section{INTRODUCTION}

High-Entropy Alloys (HEAs) are multi-component alloys forming highly disordered solid solution phases [14]. Since their discovery, just over a decade ago, HEAs have attracted the interest of the scientific community, for promising properties and potential applications (see [5-8] as well as Ref. 9 and references therein). The term HEAs, and related terms such as Multiple Principle Element Alloys [3] and Complex Concentrated Alloys [4] often refer to similar alloying concepts. While there may be ongoing discussions in the literature regarding these terms, the approach outlined here is equally applicable to any of these classifications. For the sake of brevity, only the acronym HEAs will be used throughout this article. The ongoing search to discover new high-entropy systems has recently expanded beyond metals to include entropy stabilized ceramics such as high-entropy oxides and carbides [10]. At the time of the discovery, it was conjectured that configurational entropy was the stabilizing mechanism and that many multi-component alloys would form a single phase solid solution. However, further attempts have shown that this is valid only for a fraction of multi-component alloys, while the rest form multiple phases [11]. Therefore, the key factors governing the formation of single phase HEAs remain unknown [12].

Several semi-empirical methods have been proposed to predict which multi-component alloys will form a solid solution (see Ref. 13 for an extensive review). Most approaches use descriptors as screening tools [14-20] with parameters fitted to the available, yet limited, experimental data. Modeling phase diagrams by using CALPHAD has also been applied [3, 21, 22], and it also suffers

\footnotetext{
*stefano@duke.edu
}

from insufficient experimental data. Consequently, robust prediction of solid solution forming ability in multicomponent alloys remains a major challenge hindering further HEA discovery.

Phase diagram construction of multi-component alloys based on ab-initio calculations is a direct method that can compensate for unavailable experimental data (comprehensive review by Widom [23-25]). Computationally very demanding, it involves energy calculations for many configurations and the implementation of statistical mechanical models for estimating thermodynamic properties $[26,27]$. Hitherto, it is not surprising that the application of ab-initio searches for multi-component alloys has been considered unfeasible and without a predictive role in the search for new HEAs.

Here, a novel high-throughput (HT, [28]) ab-initio method is introduced - called LTVC (Lederer-ToherVecchio- Curtarolo) - incorporating energy calculations into a mean field statistical mechanics model, and making use of order parameters for predicting the transition temperature of a multi-component system into a solid solution phase. The idea is the following: i. The AFLOW [29-36] set of repositories [37-40] for ab-initio calculations are leveraged to train cluster expansion (CE) $[26,41]$ models, within the Alloy Theoretic Automated Toolkit (ATAT) [42] and estimate zero temperature energies of atomic configurations, which are derivative structures of either fcc or bcc lattices, on which HEAs show solid solution forming ability. ii. Next, these atomic configurations are incorporated into a mean field statistical mechanical model, named the generalized quasi-chemical approximation (GQCA) [43, $\overline{4} 4]$, which is particularly suitable when long-range order is not important and the material is spatially homogeneous, as expected for solid solutions. iii. Finally order parameters are proposed to detect order-disorder phase transitions by following the evolution of the statistical population of ordered configurations. 
The predictive capability of LTVC is corroborated by Monte Carlo simulations, experimental data for binary alloys [45], CALPHAD calculations performed with Thermo-Calc $[46,47]$ for ternary alloys, and experimental data of 17 quaternary and quinary alloys shown by experiments to form solid solutions [2, 21, 48-61].

Finally, applying LTVC to quaternary and quinary systems, numerous alloys with solid solution forming ability are identified. These predictions, inaccessible by previously suggested descriptors, show that the method could become an effective guiding tool for HEA design, as well as demonstrate the importance of short-range order in these systems.

\section{METHODS}

Generation of derivative structures. HEAs form single-phase solid solutions mostly on fcc and bcc lattices [13]: the starting point is the generation of inequivalent atomic decorations of those lattices. A group-theoretical approach $[62,63]$ is used to generate a complete set of inequivalent atomic configurations with up to 8 atoms and 5 species per primitive cell (Table I), and the multiplicity (degeneracy, number of symmetrically equivalent configurations) of each configuration is calculated. The algorithm is validated with the binary and ternary configurations generated by the mmaps code of the ATAT package [64].

\begin{tabular}{|c||c|c|c|c|}
\hline at./cell & binary & ternary & quaternary & quinary \\
\hline \hline 2 & 2 & - & - & - \\
\hline 3 & 6 & 3 & - & - \\
\hline 4 & 19 & 39 & 19 & - \\
\hline 5 & 28 & 81 & 108 & 54 \\
\hline 6 & 80 & 550 & 1,360 & 1,500 \\
\hline 7 & 104 & 933 & 3,876 & 7,600 \\
\hline 8 & 390 & 6,312 & 38,372 & 111,915 \\
\hline
\end{tabular}

TABLE I. Number of inequivalent atomic configurations for fcc and bcc derivative structures including species permutations.

Calculation of zero temperature energies. The AFLOW.org repositories comprise calculated energies of relaxed atomic configurations, which are fcc and bcc derivative structures for most binaries and many ternaries. All ab-initio energies are obtained using the VASP software [65] within the AFLOW high-throughput framework [29-36] and using the standardized set of parameters [39]. The vibrational formation enthalpy is usually much smaller than the configurational contribution, especially in highly disordered systems. This allows us to neglect phonon spectra characterization, a daunting challenge for the millions of alloy-structures under investigation $[66,67]$. Complete information about these calculations is included in the open access AFLOW.org materials data repository [37-40]. For each alloy system, AFLOW energies are used as input for the mmaps code of the ATAT package. Cluster expansion is performed and energies of all configurations with up to 8 atoms per primitive cell are estimated (see binary example in Figure 1(a)). In addition, mmaps outputs the cross validation (CV) score, which is a measure for the uncertainty of predicted energies not included in the training set (lower CV score implies less uncertainty). In this article, thermodynamic analysis is performed only for systems whose CV score is less than $50 \mathrm{meV}$.

Implementation of the GQCA model. The atomic configurations are incorporated into a statistical mechanical mean field model, named the generalized quasichemical approximation (GQCA) [43, 44]. This model fits well to high-throughput workflows, as the number of degrees of freedom varies linearly with the number of atomic types in the cell (compared to the cluster variation method $[26,68]$, whose number of degrees of freedom grows rapidly with cluster size).

The model factorizes a parent lattice of $N$ sites, hosting $K$ species, into non-overlapping space-filling cells of equal number of sites. The clusters are treated as independent, and the total energy of the $N$-site lattice is approximated as a sum of the $n$-atom cell energies, each calculated separately. Here, the factorization is implemented using 8atom cells, and the energy per site of each cell is estimated by assuming periodic boundary conditions on the cell surfaces: each cell has the energy and multiplicity (symmetry degeneracy) of an analog periodic ordering, having $1,2,4$, or 8 atoms per primitive cell. This implementation suits well the description of ordered or disordered phases having the same parent lattice type, as long as order effects can be captured within 8-atom cells and the spatial homogeneity assumption remains valid (justifying neglect of boundary interactions between phases). In the high- $T$ limit, a fully disordered phase (with ideal entropy of mixing) is represented by cells randomly populating the parent lattice according to the multiplicities. The summation of cell energies over a random distribution to obtain the total energy reduces a potential systematic error, related to the assumption of periodic boundary conditions. In the low- $T$ limit, ordered compounds or elemental phases are trivially demonstrated when only one type of unit cell (periodic ordering) occupies the whole $N$-site parent lattice. Then, the total energy exactly becomes the sum of the cell's energies. In this article, the GQCA model is implemented only for fcc or bcc type parent lattices. The incorporation of other ordered compounds, not of fcc or bcc type, into the thermodynamic analysis is described below in point iv in the section on "Order-disorder transition".

Within the stated assumptions on the GQCA formalism (see Refs. 43 and 44 for details), the thermodynamic potential becomes

$$
\Phi=N \cdot\left(\sum_{j} \epsilon_{j} P_{j}-T s-\sum_{k} \mu_{k} X_{k}\right),
$$

where $\left\{P_{j}\right\}$ is the probability distribution of finding the 
a

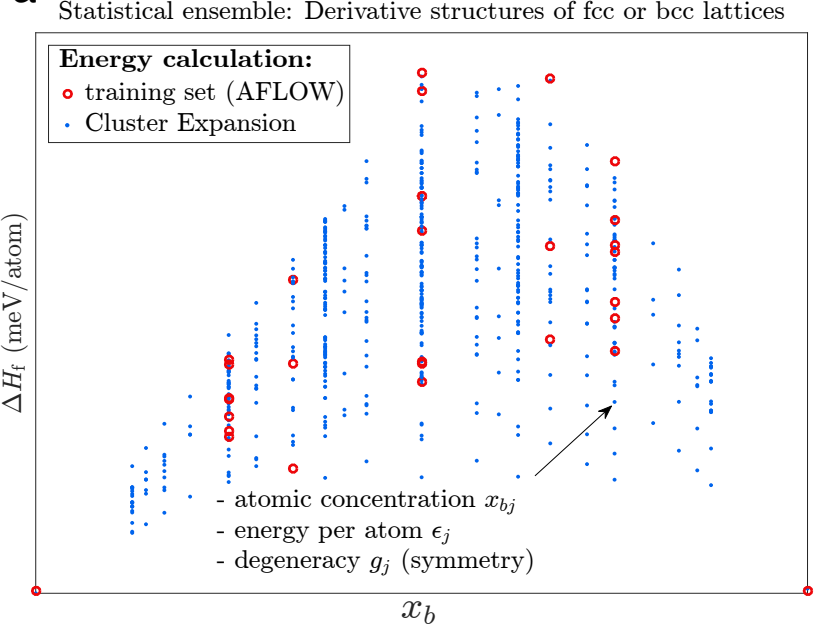

C

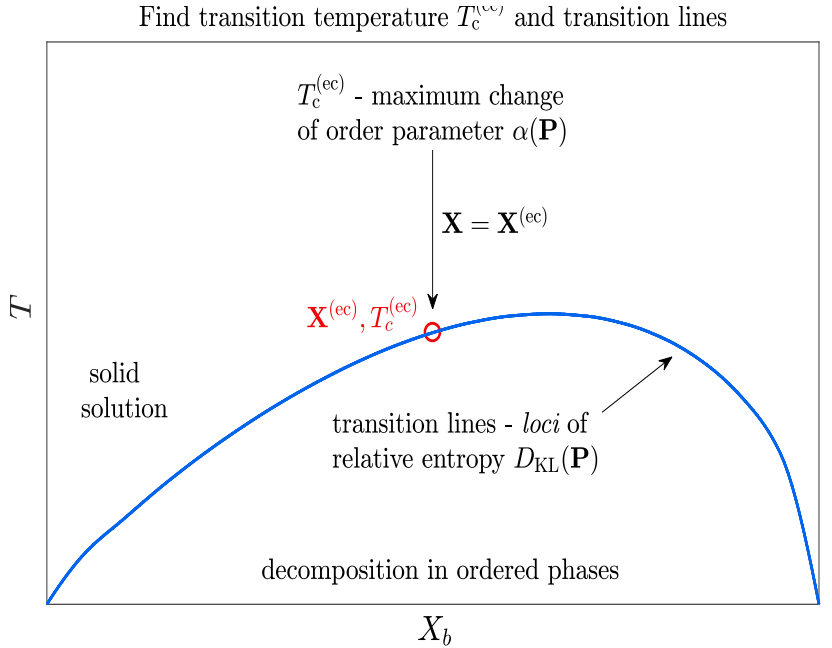

b Solve model equations for the population vector $\mathbf{P}$

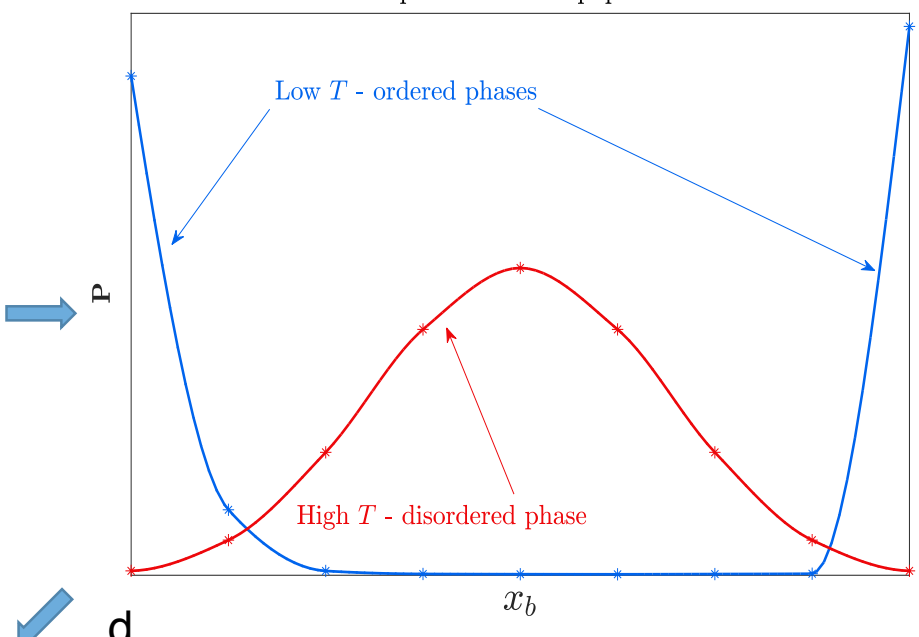

d

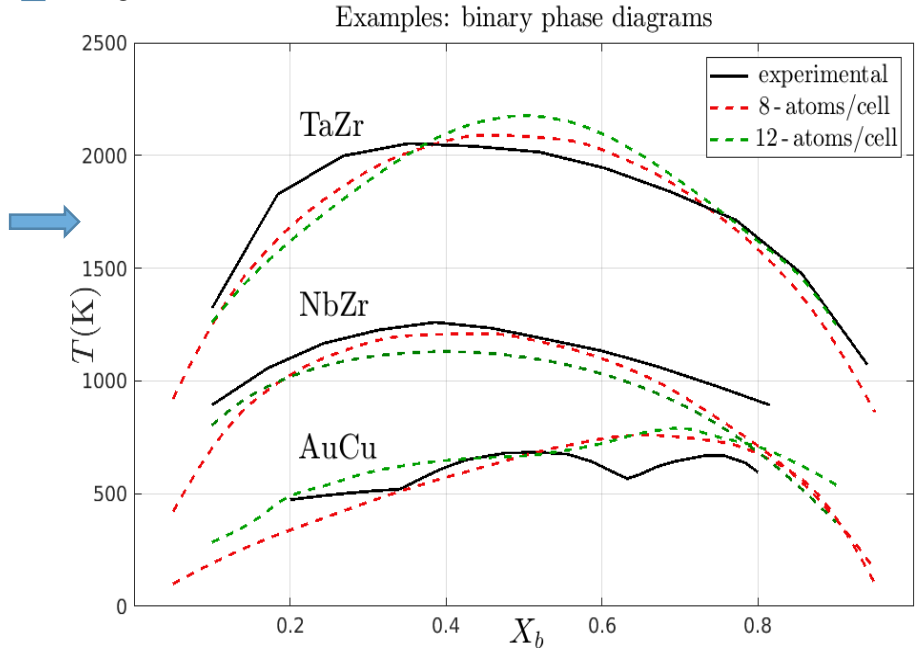

FIG. 1. Outline of the LTVC method. (a) Statistical ensemble construction: atomic configurations are generated, multiplicities are calculated, and energies are estimated using cluster expansion trained by the AFLOW dataset. (b) The statistical mechanical model is solved for the population vector $\mathbf{P}(\mathbf{X}, T)$ (Eq. (6)). The high- $T$ limit represents a fully disordered phase (Eq. (3)), and the low- $T$ phase separation or ordered compound formation. (c) $T_{\mathrm{c}}$ at equi-composition is identified with maximal change of the order parameter $\alpha$ (Eq. (9)). Transition lines are traced as the loci of the relative entropy, $D_{\mathrm{KL}}$ (Eq. (4)). (d) Examples of calculated binary phase diagrams, using a factorization of 8-atom cells (dashed red lines) and 12-atom cells (dashed green lines). Solid black lines represent experimental results, extracted from Ref. [45].

cells with $n=8$ atoms in the $N$-site lattice, $X_{k}$ represents the macroscopic atomic concentration of $k$-species atoms on the $N$-site lattice, and $\epsilon_{j}, s$ and $\mu_{k}$ are the energy of $j^{\text {th }}$ cell, configurational entropy and the chemical potentials per atom, respectively. The large $N$ limit for the entropy is

$$
s=k_{\mathrm{B}}\left(-\sum_{k} X_{k} \log \left(X_{k}\right)+\frac{1}{n} \sum_{j} P_{j} \log \left(P_{j} / \tilde{P}_{j}\right)\right),
$$

where $k_{\mathrm{B}}$ is the Boltzmann constant and $\left\{\tilde{P}_{j}\right\}$ is the $T$ independent random probability distribution of finding the cells in the $N$-site lattice,

$$
\tilde{P}_{j}=\frac{g_{j} \prod_{k} X_{k}^{n \cdot x_{k j}}}{\sum_{j^{\prime}} g_{j^{\prime}} \prod_{k} X_{k}^{n \cdot x_{k j^{\prime}}} .}
$$

Here, $g_{j}$ is the multiplicity of the $j^{\text {th }}$ cell and $x_{k j}$ is the fraction of $k$-species atoms. The first term in Eq. (2) represents the ideal entropy of mixing. The second term,

$$
D_{\mathrm{KL}}(\mathbf{X}, T) \equiv \frac{1}{n} \sum_{j} P_{j} \log \left(P_{j} / \tilde{P}_{j}\right),
$$

is known as the relative entropy or the Kullback-Leibler divergence [69], and is commonly used to quantify entropy loss due to ordering [70-72]. Minimizing the thermody- 

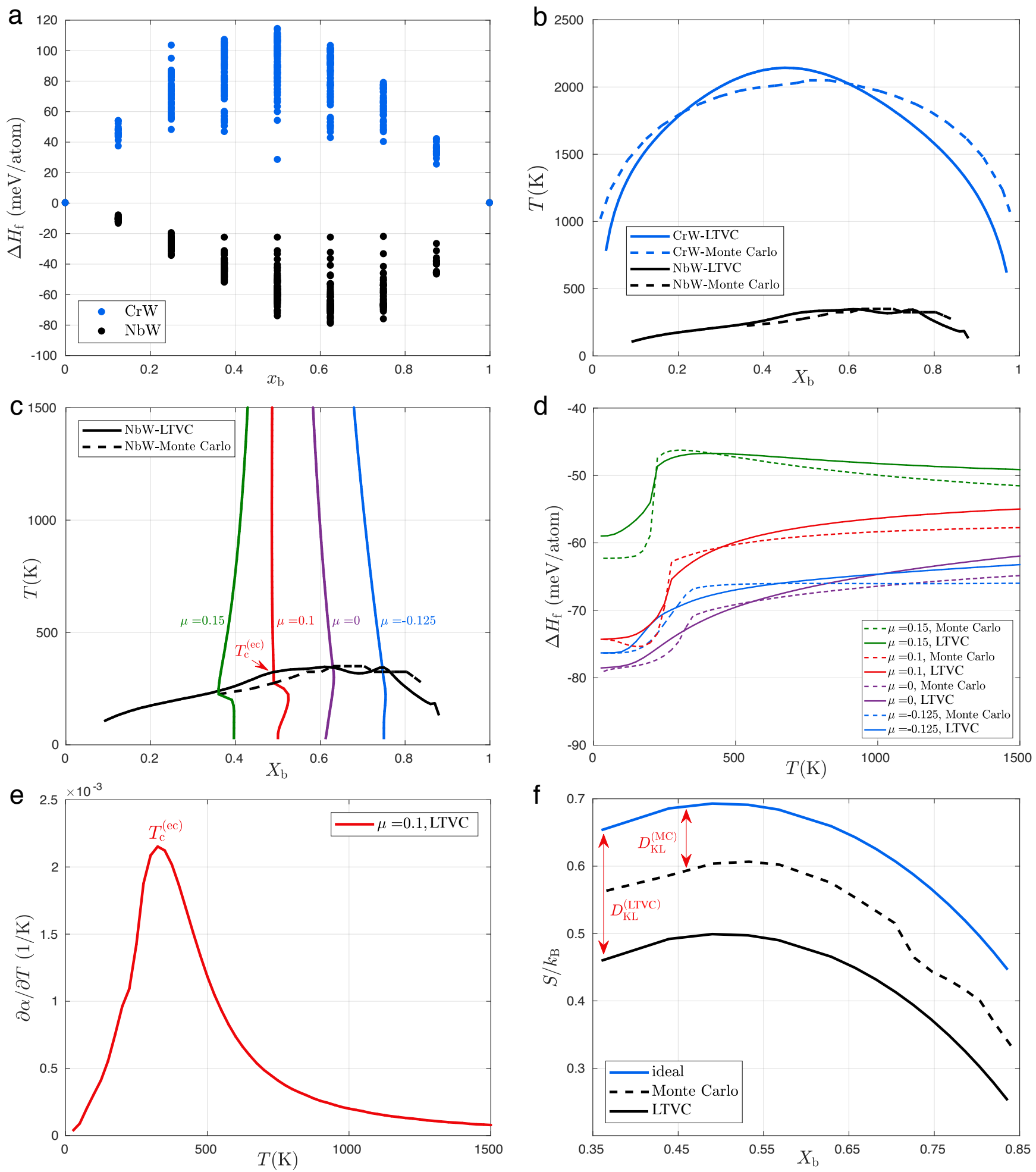

FIG. 2. Comparison between LTVC and Monte Carlo simulations. (a) Zero temperature formation enthalpies for CrW (blue) and $\mathrm{NbW}$ (black) from cluster expansion, which also drive the Monte Carlo calculations. (b) Transition lines for CrW (blue lines) and NbW (black lines), computed using LTVC (solid lines) and Monte Carlo simulations (dashed lines). (c) The $\mathrm{NbW}$ transition lines, with a sample of constant chemical potential trajectories, taken from the Monte Carlo simulation. (d) The NbW formation enthalpies along the $(\mathbf{X}, T)$, constant chemical potential trajectories, computed using LTVC (solid lines) and Monte Carlo simulations (dashed lines). (e) Temperature derivative of the order parameter $\alpha$, Eq. (9), for the NbW case. The equi-composition transition temperature, $T_{\mathrm{c}}^{(\mathrm{ec})}$, is identified as the point where $\partial \alpha / \partial T$ reaches its maximum value. (f) Entropy, $k_{\mathrm{B}}$ normalized, across the order-disorder transition lines, computed using LTVC (solid black line) and Monte Carlo simulations (dashed black lines) for the NbW case. The blue line indicates the ideal mixing entropy limit. $D_{\mathrm{KL}}^{(\text {model })}$ and $D_{\mathrm{KL}}^{(\mathrm{MC})}$ mark the relative entropy reduction, Eq. (4), computed using LTVC and Monte Carlo simulations, respectively. The near constant behavior of $D_{\mathrm{KL}}^{(\mathrm{MC})}$ supports the method's assumption. 
namic potential, subject to the constraints

$$
\sum_{j} P_{j}=1 ; \quad \sum_{j} P_{j} x_{k j}=X_{k}
$$

the equilibrium probability distribution is found $(\beta=$ $\left.1 / k_{\mathrm{B}} T\right)$ :

$$
P_{j}=\frac{\tilde{P}_{j} e^{-n \beta\left(\epsilon_{j}-\sum_{k} \mu_{k} x_{k j}\right)}}{\sum_{j} \tilde{P}_{j} e^{-n \beta\left(\epsilon_{j}-\sum_{k} \mu_{k} x_{k j}\right)}} .
$$

The expression for the probability distribution is reinserted into Eq. (5) for the $(K-1)$ independent $\mu_{k}(\mathbf{X}, T)$ functions of temperature and macroscopic concentration $^{1}$. The thermodynamic potential, Eq. (1), and its related thermodynamic quantities are then easily obtained as functions of temperature and macroscopic concentration.

Order-disorder transition. The random population vector $\tilde{\mathbf{P}}$, Eq. (3), is the high- $T$ limit of population vector $\mathbf{P}$, Eq. (6). The limit represents a fully disordered phase with ideal entropy of mixing (only the first term in Eq. (2) being non-zero, while the relative entropy vanishes). An order-disorder transition locus, $\tilde{T}_{\mathrm{c}}(\mathbf{X})$, can be found as the intersection between the potential of a fully disordered phase

$$
\tilde{\Phi}_{\text {disorder }}(\mathbf{X}, T) \equiv \lim _{\mathbf{P} \rightarrow \tilde{\mathbf{P}}} \Phi(\mathbf{X}, T),
$$

with that of a competing ordered phase, $\Phi_{\mathrm{order}}^{\mathrm{AFLOW}}(\mathbf{X})$, estimated by using the AFLOW convex-hull database [73],

$$
\tilde{\Phi}_{\text {disorder }}\left(\mathbf{X}, \tilde{T}_{\mathrm{c}}\right)=\Phi_{\text {order }}^{\text {AFLOW }}(\mathbf{X}) \Rightarrow \tilde{T}_{\mathrm{c}}(\mathbf{X}) .
$$

The approach, equivalent to a common-tangent construction of the two asymptotic free energies, is straightforward. Unfortunately, it disregards the effect of short range order on both the energy and entropy of the disordered phase, potentially leading to erroneous transition temperatures.

To overcome this issue, a two-step algorithm (marked i-ii) is proposed. In spirit, it is similar to what is done for phase-diagram construction using Monte-Carlo simulations, where an order parameter locating an orderdisorder transition is followed by an algorithm tracing the boundary line.

i. Estimation of the order-disorder transition temperature at equi-composition. The change of the population vector from the high- $T$ limit (representing full disorder), to the low- $T$ limit (representing ordered compounds or elemental phases), motivates the introduction of the order parameter:

$$
\alpha(\mathbf{X}, T) \equiv \mathbf{P} \cdot \tilde{\mathbf{P}} /|\mathbf{P} \| \tilde{\mathbf{P}}|
$$

\footnotetext{
${ }^{1}$ vector $\mathbf{X}$ represents the macroscopic concentrations $X_{k}$
}

which measures the deviation of the population vector from the high- $T$ limit. Similar to the behavior of the order parameter in Monte-Carlo simulations [27], maximal change is expected at order-disorder transition (see Figure 2(e)). The transition temperature at equicomposition $T_{\mathrm{c}}^{(\mathrm{ec})}$ is identified as the point where $\partial \alpha / \partial T$ reaches its maximum value (red circle in Figure 1(c)).

ii. Tracing the boundary lines. Near the solid solution boundary lines, the entropy of the disordered phase decreases due to the growth of short range order. The boundary lines separating solid solutions and decomposition into ordered phases (blue line in Figure 1(c)) are found, assuming entropy reduction is approximately constant across them

$$
D_{\mathrm{KL}}\left(\mathbf{X}, T_{\mathrm{c}}\right) \approx D_{\mathrm{KL}}\left(\mathbf{X}^{(\mathrm{ec})}, T_{\mathrm{c}}^{(\mathrm{ec})}\right) .
$$

Here, $D_{\mathrm{KL}}$ is the relative entropy, defined in Eq. (4), $T_{\mathrm{c}}^{(\mathrm{ec})}$ is the transition temperature at equi-concentration, $\mathbf{X}^{(\mathrm{ec})}$, and $T_{\mathrm{c}}$ is the transition temperature at $\mathbf{X}$. The approximation works well for alloys characterized by relatively simple phase diagrams (see Figure 2(f)). For more complex systems, involving the formation of multiple ordered phases at low temperatures (as predicted by the AFLOW convex hull), a more accurate method should involve a detailed study of the population vector $\mathbf{P}(\mathbf{X}, T)$ at low temperatures. Since HEAs usually form near equicomposition, step ii is not critical, and the results reported are based on the use of only $\alpha$ as the order parameter.

iii. Predicting the solid solution parent lattice. Steps i-ii are independently implemented for fcc and bcc lattices. The lowest thermodynamic potential, Eq. (1), will determine the structure of the solid solution.

iv. Effect of other competing ordered phases. The formation of ordered phases, not of fcc or bcc type, might raise the order-disorder transition temperature in some parts of the phase diagram. Using the AFLOW convexhull, the minimal thermodynamic potential of all other ordered phases $\Phi_{\text {order }}^{\text {AFLOW }}(\mathbf{X})$ is estimated, leading to a potentially higher transition temperature:

$$
\Phi\left(\mathbf{X}, T_{\mathrm{c}}^{\mathrm{gs}}\right)=\Phi_{\text {order }}^{\text {AFLOW }}(\mathbf{X}) \Rightarrow T_{\mathrm{c}}^{\mathrm{gs}}(\mathbf{X}) .
$$

If $T_{\mathrm{c}}^{\mathrm{gs}}$ is higher than $T_{\mathrm{c}}$ found by steps $\mathbf{i}$-ii-iii, then $T_{\mathrm{c}}^{\mathrm{gs}}$ is used as a better estimate for the transition temperature $T_{\mathrm{c}}$.

Test of cell size factorization convergence. Figure 1(d) compares calculated with experimental boundary lines, taken from Massalski et al. [45]. To test LTVC's convergence with respect to cell size, calculations are performed using 8-atom cells and 12-atom cells (dashed red and green lines, respectively). Similarity between calculated and experimental boundaries demonstrates the method's ability to follow order-disorder transitions at equi-composition when either phase separation (NbZr and $\mathrm{TaZr}$ ) or ordered compound formation $(\mathrm{AuCu})$ oc- 
cur at low temperatures.

Comparison of the LTVC method versus Monte Carlo simulations. Monte Carlo (MC) simulations are accurate — albeit computationally demanding — tools for calculating thermodynamic properties. LTVC and MC results are compared. The latter are obtained with the ATAT-memc2 code [27]. Comparisons provide direct and reliable benchmarks as the two approaches are driven by the same cluster expansion. Two representative alloys were chosen: $\mathrm{CrW}$ and $\mathrm{NbW}$. Figure 2(a) shows the zero temperature formation enthalpies of $\mathrm{CrW}$ (blue) and $\mathrm{NbW}$ (black), as obtained from a cluster expansion on a bcc parent lattice. The two alloys exhibit very different energy landscapes. The positive formation enthalpies of CrW leads to phase separation to elemental phases at low temperatures, while the negative formation enthalpies of NbW promote ordered compounds.

Figure 2(b) shows the order-disorder transition lines of the two alloys, using LTVC (solid lines) and MC (dashed lines). For a wide concentration range, the agreement demonstrates the ability of the method to accurately reproduce order-disorder transitions when ordered compound formation or phase separation appear at low temperature.

Figures 2(c-f) depicts the NbW case. Panel (c) shows a sample of constant chemical potential trajectories, obtained from the MC simulations. Panel (d) compares the formation enthalpy along such $(X, T)$ trajectories, using LTVC (solid lines) and MC simulations (dashed lines). The agreement demonstrates the ability of the method to accurately estimate the Gibbs free energy of the alloy both at high (disorder) and low temperatures (order).

Figure 2(e) shows the temperature derivative of the order parameter $\alpha$ (Eq. (9)) along the equi-composition trajectory $(\mu=0.1$ red line in Figure 2(c)). The sensitivity of $\alpha$ to the underlying phase transition allows easy identification of $T_{\mathrm{c}}^{(\mathrm{ec})}=325 \mathrm{~K}$, close to the MC prediction (275K). Panel (f) depicts the entropy estimated by LTVC and the MC simulations along their respective boundary lines. First, despite $D_{\mathrm{KL}}^{(\mathrm{MC})}$ - the entropy reduction from the ideal mixing entropy limit (blue line) — being smaller than $D_{\mathrm{KL}}^{\text {(model) }}$, Eq. (4), the model predictions do not depend on the absolute value of $D_{\mathrm{KL}}$ at $T_{\mathrm{c}}^{(\mathrm{ec})}$, so the difference is irrelevant. Second, the small variation of $D_{\mathrm{KL}}^{(\mathrm{MC})}$ justifies the working assumption about the transition lines as the loci of relative entropy, Eq. (10). In conclusion, LTVC's combination of order parameter $\alpha$ and the relative entropy $D_{\mathrm{KL}}$ leads to boundaries very similar to those obtained from MC.

\section{RESULTS}

Binary alloys. The experimental data for 117 binary alloys is collected [45] and compared with the predictions. For each system, cluster expansion is performed separately on bcc and fcc lattices. The energies of all inequivalent atomic configurations with up to 8 atoms per primitive cell (631 for each lattice) are estimated. Thermodynamic analysis is then performed as described above and the transition temperature $T_{\mathrm{c}}$ at equi-composition is estimated. The standard deviation of $T_{\mathrm{c}}$ is estimated as follows: zero temperature energies, predicted by cluster expansion, are randomly shifted according to a normal distribution. The standard deviation of the normal distribution is the CV score, retrieved from the output of the mmaps code of ATAT. Thermodynamic analysis is performed repeatedly, each time with new shifted energies, until convergence of the standard deviation of $T_{\mathrm{c}}$ is obtained. A solid solution is predicted if $T_{\mathrm{c}}<T_{\mathrm{m}}^{(\exp )}$, where $T_{\mathrm{m}}^{(\exp )}$ is the experimental melting temperature at the appropriate composition. The results are presented in Figure 3 and listed in Table III.

- Existence. Formation (or not) of the solid solution is correctly reproduced in 102 out of 117 analyzed systems with an effectiveness of $\eta_{\mathrm{ss}}=102 / 117=87.2 \%$.

- Solid solution forming systems. 56 out of the 58 predicted solid solution-forming are experimentally corroborated with correct underlying lattice: $\eta_{\text {latt }}=56 / 58=96.6 \%$. In such cases, LTVC tends to overestimate the transition temperature by $\sim 11 \%$.

- Non solid solution forming systems. 46 out of 59 systems predicted not to form a solid solution phase are experimentally verified as such. Erroneous prediction of 9 out of 13 alloys is explained by the estimated standard deviation of $T_{\mathrm{c}}$.

- Three binaries (AuNi, MnNi, MnV) exhibit differences greater than 3 standard deviations between calculated and experimental transition temperatures. Test Monte Carlo simulations, performed using ATAT [27], predict $T_{\mathrm{c}}$ values similar to those of LTVC. Likely, the problem is related to the neglect of vibrational formation entropy coming from phonon contributions $(\mathrm{AuNi}$, one of these three alloys, is known to have large vibrational entropy of formation [74]), or to insufficient training data: the zero temperature formation enthalpy calculations (performed on primitive cells with 4 atoms or less) could be incommensurate with larger-size order effects (e.g. magnetic), and thus effectively overestimate the transition temperatures.

- The two approaches $\tilde{T}_{\mathrm{c}}$, Eq. (8), and $T_{\mathrm{c}}$, steps i-iv, produce similar results, indicating that for binaries the effect of short range order is limited. Later, we will show that this will not be the case for ternaries.

Ternary alloys. Next, the complete set of 4,495 ternary alloys that can be formed from 31 species is addressed ${ }^{2}$. In the absence of an extensive database of experimentally studied ternary alloys, the predictions of the LTVC

\footnotetext{
$2 \mathrm{Ag}, \mathrm{Al}, \mathrm{As}, \mathrm{Au}, \mathrm{Co}, \mathrm{Cr}, \mathrm{Cu}, \mathrm{Fe}, \mathrm{Ge}, \mathrm{Hf}, \mathrm{Ir}, \mathrm{Mn}, \mathrm{Mo}, \mathrm{Nb}, \mathrm{Ni}$, Os, Pd, Pt, Re, Rh, Ru,Sb, Sc, Si, Ta, Tc, Ti, V, W, Y, and Zr.
} 


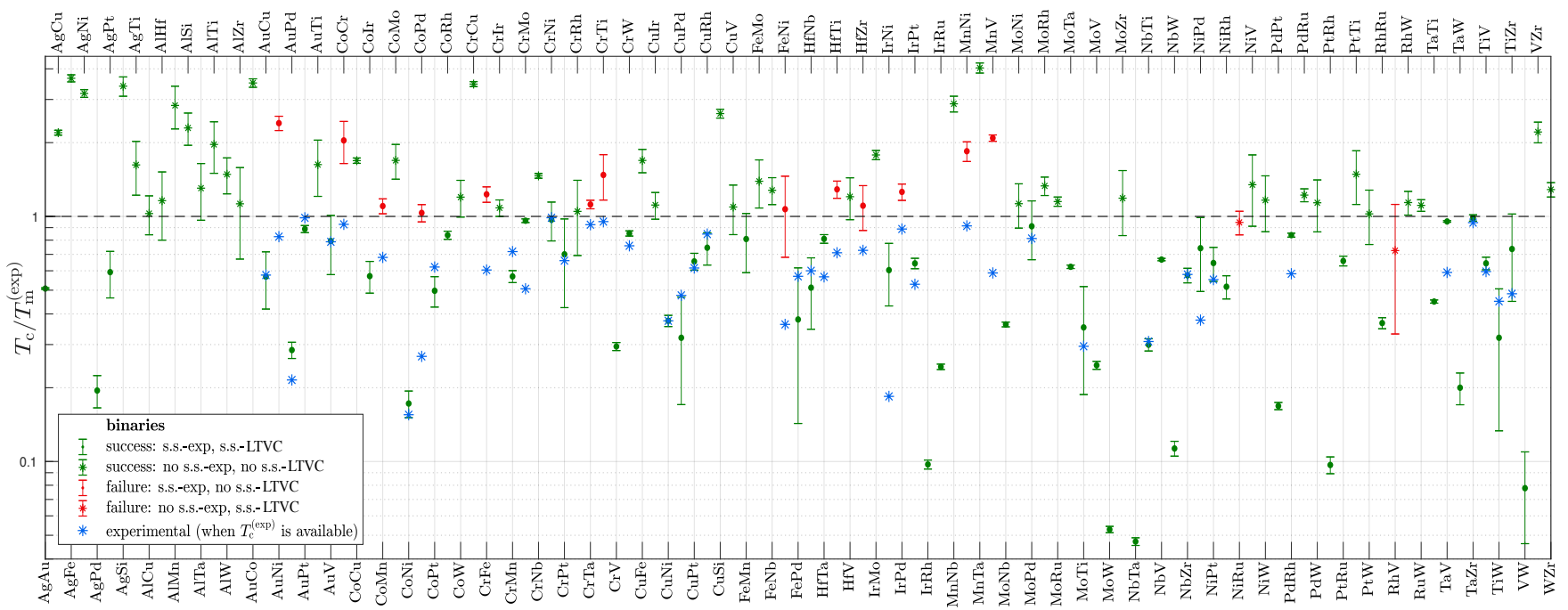

FIG. 3. $T_{\mathrm{c}} / T_{\mathrm{m}}^{(\mathrm{exp})}$ and success rate for 117 binary alloys (alphabetic order). A solid solution is predicted by LTVC if $T_{\mathrm{c}}<T_{\mathrm{m}}^{(\mathrm{exp})}$ (points below the dashed line). Success rate of $87.2 \%$ is calculated as ratio of successes (in green) to total [successes+failures]; (failures in red).

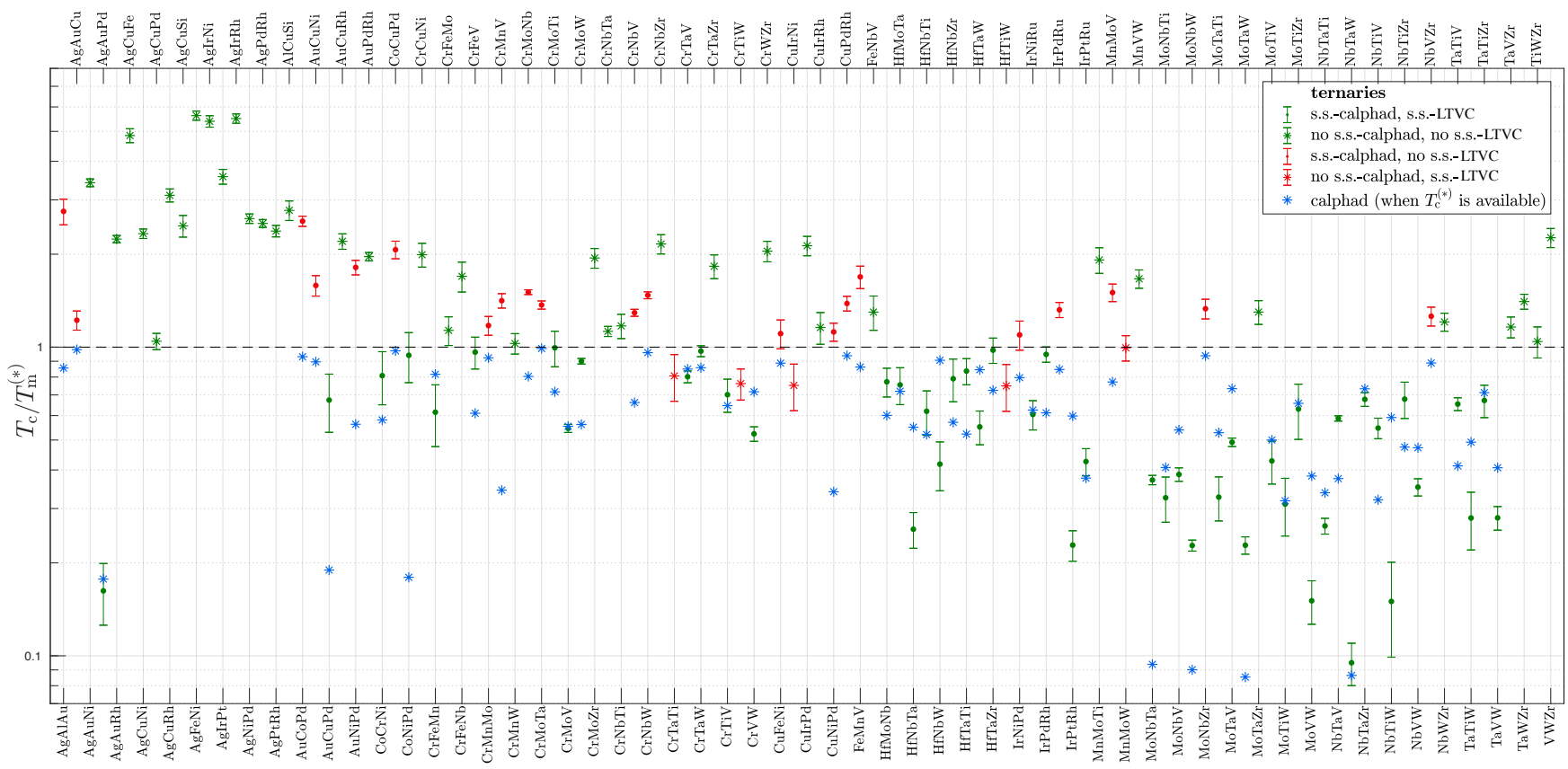

FIG. 4. $T_{\mathrm{c}} / T_{\mathrm{m}}^{(*)}$ and comparison with CALPHAD predictions for 113 ternary alloys (alphabetic order). $T_{\mathrm{m}}^{(*)}$ is the solidus temperature taken from CALPHAD. A solid solution is predicted by LTVC if $T_{\mathrm{c}}<T_{\mathrm{m}}^{(*)}$ (points below the dashed line). Success rate of $77.0 \%$ is calculated as ratio of successes (in green) to total [successes +failures]; (failures in red).

method are compared with the results of calculations employing the CALPHAD methodology [3, 21, 22].

i. CALPHAD. Phase diagram calculations are performed using the Thermo-Calc software and the SSOL5 database for binary and ternary alloys [46, 47]. The following parameters are assessed at equi-composition: the solidus temperature, $T_{\mathrm{m}}^{(*)}$, the number and types of phases at this temperature, the first reaction temperature below $T_{\mathrm{m}}^{(*)}$ and the number and types of phases below the first reaction temperature. Formation (or not) of a solid solution is then tested, along with its underlying lattice and the critical temperature, $T_{\mathrm{c}}^{(*)}$. Although these predictions are based mainly on extrapolation of binary data, they are expected to be reasonably reliable, as long as the associated binaries of each ternary alloy are included in the SSOL5 database. Cases where the solid solution phase found by Thermo-Calc is of hcp type are not included in the comparison (as ab-initio modeling of a solid 

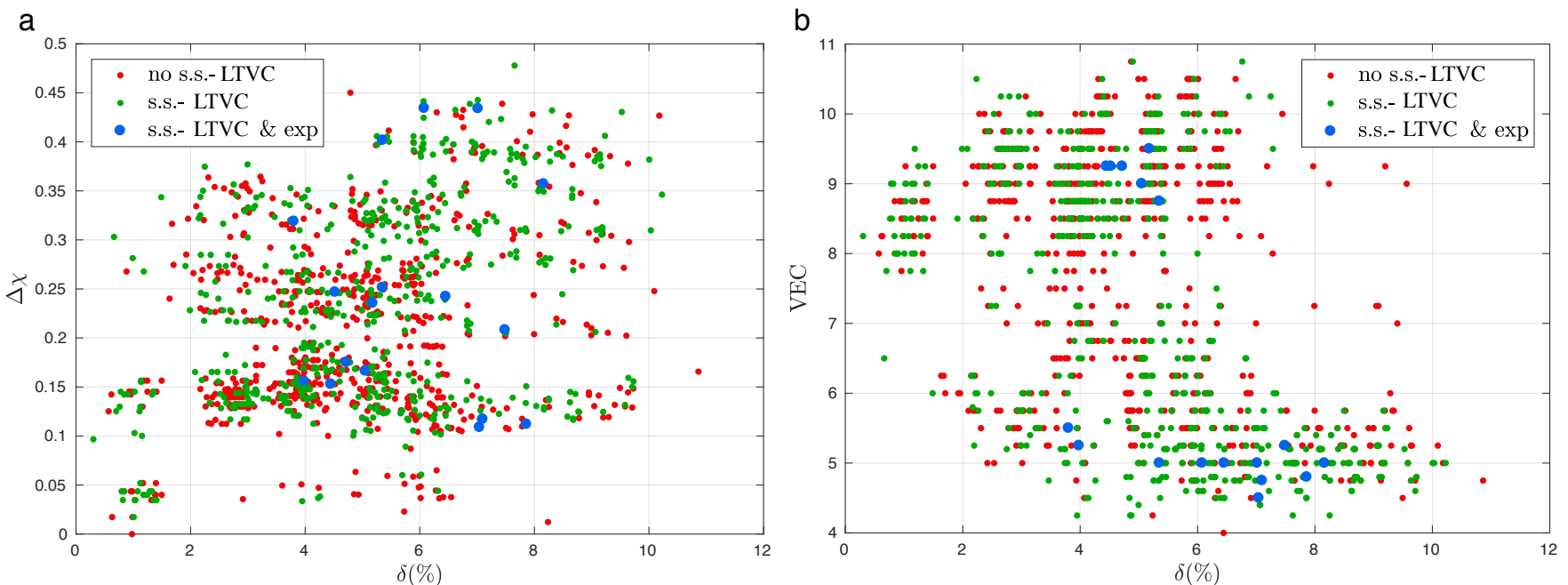

FIG. 5. The failure of empirical parameters, suggested as descriptors for HEAs, to pinpoint solid solutions. Model predictions for quaternary and quinary alloys (green - predicted s.s., blue - predicted s.s. and verified by experiments, see Table II) are plotted as a function of: (a) the electro-negativity difference $(\Delta \chi)[15]$ and the atomic size difference $(\delta)$ [14]; (b) the valence electron concentration (VEC) [19] and the atomic size difference $(\delta)$.

solution phase was not performed on this lattice).

ii. Ab-initio method. As for binaries, cluster expansion is performed for each ternary alloy on bcc and fcc lattices using the available training data of the AFLOW repositories. Cluster expansion is performed and the energies of all inequivalent configurations ( 9,808 for each lattice) are estimated. Thermodynamic analysis is performed for ternaries whose CV score is less than $50 \mathrm{meV}$ and their transition temperature $T_{\mathrm{c}}$ at equi-composition is obtained, along with its estimated standard deviation. A solid solution is predicted if $T_{\mathrm{c}}<T_{\mathrm{m}}^{(\star)}$.

Results of ab-initio and CALPHAD for 441 ternaries are listed in Table IV. Figure 4 shows the results for 113 ternaries whose CV score is less than $30 \mathrm{meV}$ and the 3 associated binaries of each ternary alloy are found in the SSOL5 database.

- Existence. Formation (or not) of the solid solution is in agreement for 87 out of 113 ternaries with an effectiveness of $\eta_{\mathrm{ss}}=87 / 113=77.0 \%$.

- Solid solution forming systems. 49 out of the 54 predicted solid solution-forming cases are corroborated by CALPHAD with correct underlying lattice: $\eta_{\text {latt }}=49 / 54=90.7 \%$. The transition temperatures of these 49 ternaries are in good agreement. In such cases, the method predicts transition temperatures slightly higher $(\sim 3 \%)$ than CALPHAD. Formation of solid solutions is not corroborated by CALPHAD in 5 out of 54 cases. Here, the disagreement between the two methods can be related to the standard deviation of $T_{\mathrm{c}}$ as well. Note that for 4 of these 5 cases, CALPHAD exhibits the same solid solution phase below $T_{\mathrm{m}}^{(*)}$, accompanied by an additional phase.

- Non solid solution forming systems. 38 out of 59 nonforming predicted cases are corroborated by CALPHAD. Out of the 21 cases in disagreement, 15 are not explained by the standard deviations of $T_{\mathrm{c}}$ (differences are of greater than 3 standard deviations). As for binaries, the problem could be related to the fact that the LTVC method neglects the vibrational entropy of formation, or to insufficient training data for cluster expansion calculations.

- $\tilde{T}_{\mathrm{c}}$ values (approximated according to Eq. (8)) are lower than $T_{\mathrm{c}}$ by $38.3 \%$ and 20 of its 86 predicted solid solution-forming cases are in disagreement with CALPHAD. This shows that the effect of short range order on transition temperature is non-negligible for ternaries.

Search for quaternary and quinary HEAs. As before, for each alloy, cluster expansion is performed separately on bcc and fcc lattices, based on the available training data of the AFLOW repositories. The energies of all inequivalent configurations $(79,185$ for quaternaries, 425,219 for quinaries) are estimated. Thermodynamic analysis is performed for alloys whose CV score is less than $50 \mathrm{meV}$, and the transition temperature $T_{\mathrm{c}}$ at equicomposition is obtained. The results for 1110 quaternary alloys and 130 quinary alloys (mainly those based on the refractory elements $\mathrm{Cr}, \mathrm{Hf}, \mathrm{Mo}, \mathrm{Nb}, \mathrm{Ta}, \mathrm{Ti}, \mathrm{V}, \mathrm{W}$ and $\mathrm{Zr}$ ) are listed in Tables V and VI, respectively. 571 of the 1240 quaternaries and quinaries show solid solution forming ability. These numbers demonstrate that among multicomponent alloys, one can expect to find a plethora of solid solutions. Table II shows results for alloys, demonstrated by experiments to form solid solutions [2, 21, 4861]. The data of all experimentally studied alloys and $T_{\mathrm{c}}$ values of Monte-Carlo simulations [23, 24] corroborates the method's accuracy. $\tilde{T}_{\mathrm{c}}$ values (approximated according to Eq. (8)) are significantly lower, demonstrating again the importance of short range order effects.

As mentioned, several empirical parameters have been 
TABLE II. Comparison between LTVC and experimental data for quaternaries and quinaries. S.S. type of single phase solid solution; $T_{\mathrm{c}}(\mathrm{K})$ calculated transition temperature; $\tilde{T}_{\mathrm{c}}(\mathrm{K})$ approximated transition temperature, estimated according to Eq. (8); $\bar{T}_{\mathrm{m}}(\mathrm{K})$ melting temperature (solidus), calculated as the average of the melting temperatures at equi-composition of the binaries associated with the alloy; A solid solution is predicted if $T_{\mathrm{c}}<\bar{T}_{\mathrm{m}}$; CV (meV) cluster expansion cross validation score; S.S. Exp. type of single phase solid solution found in experiments.

\begin{tabular}{|c|c|c|c|c|c|c|}
\hline HEA & S.S. & $T_{\mathrm{c}}$ & $\tilde{T}_{\mathrm{c}}$ & $\bar{T}_{\mathrm{m}}$ & $\mathrm{CV}$ & S.S. Exp. \\
\hline $\mathrm{CoCrFeNi}$ & fcc & 1,600 & 1,000 & $1,720^{\mathrm{a}}$ & 47 & fcc $[48,49]$ \\
\hline $\mathrm{CoCrMnNi}$ & fcc & 1,280 & 1,020 & 1,580 & 37 & fcc $[48]$ \\
\hline CoFeMnNi & fcc & 1,550 & 900 & 1,600 & 48 & fcc $[21,48]$ \\
\hline AlNbTiV & bcc & 1,260 & 1,070 & 1,975 & 50 & bcc $[51]$ \\
\hline HfNbTiZr & bcc & 970 & 840 & 2,135 & 22 & bcc $[52]$ \\
\hline MoNbTaW & bcc & $1,010^{\mathrm{b}}$ & 540 & 3,065 & 4 & bcc $[53,54]$ \\
\hline NbTaTiV & bcc & 970 & 800 & 2,345 & 6 & bcc $[55]$ \\
\hline NbTiVZr & bcc & $1,400^{\mathrm{c}}$ & 1,110 & 1,955 & 16 & bcc $[56]$ \\
\hline CoCrFeMnNi & fcc & 850 & 950 & 1,640 & 43 & fcc [2] \\
\hline AlCrMoTiW & bcc & 1,650 & 1,000 & 2,150 & 44 & bcc $[58]$ \\
\hline HfNbTaTiZr & bcc & 1,300 & 750 & 2,280 & 17 & bcc $[59]$ \\
\hline HfNbTiVZr & bcc & 1,750 & 900 & 2,020 & 21 & bcc $[61]$ \\
\hline MoNbReTaW & bcc & 2,750 & 1,100 & 3,000 & 14 & bcc $[60]$ \\
\hline MoNbTaTiV & bcc & 800 & 450 & 2,470 & 8 & {$[60]$} \\
\hline MoNbTaVW & bcc & 1,000 & 500 & 2,800 & 5 & bcc $[53,54]$ \\
\hline MoNbTiVZr & bcc & 1,450 & 700 & $2,120^{\mathrm{d}}$ & 17 & bcc [57] \\
\hline NbReTaTiV & bcc & 950 & 800 & 2,470 & 19 & bcc $[60]$ \\
\hline \multicolumn{7}{|c|}{$\begin{array}{l}\mathrm{a} \quad T_{\mathrm{m}}^{(\exp )}=1,695 \mathrm{~K}[48] \\
\mathrm{b} \quad \text { Monte Carlo predicts } T_{\mathrm{c}}=1,280 \mathrm{~K}[24] \\
\mathrm{c} \text { Monte Carlo predicts } T_{\mathrm{c}}=1,250 \mathrm{~K}[23] \\
\mathrm{d} \quad T_{\mathrm{m}}^{(\exp )}=2,380 \mathrm{~K}[57]\end{array}$} \\
\hline
\end{tabular}

proposed as descriptors for single phase HEAs. Until now, they were only tested versus very limited experimental data. The new predictions serve as a much larger test set. Figure 5 shows that three of the suggested descriptors: the electro-negativity difference $(\Delta \chi)[15]$, the atomic size difference $(\delta)[14]$ and the valence electron concentration (VEC) [19] (see [11] for formulas and values used in this article for these parameters), fail to pinpoint the quaternary and quinary solid solutions predicted by LTVC. Although not presented here, it is noted that another descriptor, based on enthalpies of formation calculated for binary compounds [20], also fails to identify the alloys forming solid solutions according to LTVC. These findings challenge the view that these simple descriptors can serve as an effective search tool for single phase HEAs.

\section{CONCLUSIONS}

Robust prediction of solid solution forming ability in multi-component alloys remains a major challenge hindering the discovery of novel HEAs. This article intro- duces a novel high-throughput method - called LTVC - enabling ab-initio searches through the vast space of possible multi-component alloys of solid solutions. Based on the synergy of AFLOW repositories, cluster expansion and a straightforward, yet accurate, mean field theory model, the approach can become an effective and efficient guiding tool for HEA design.

The accuracy is corroborated by Monte Carlo simulations, experimental data for binaries ( $87.2 \%$ agreement), CALPHAD calculations for ternaries $(77.0 \%$ agr. $)$ and experimental data for 17 quaternary and quinary alloys (100\% agr.). Solid solution-forming cases are confirmed with high success rate: $96.6 \%, 90.7 \%, 100 \%$ and $100 \%$ for binaries, ternaries quaternaries and quinaries, respectively. The underlying lattice of the solid solution is correctly predicted as well. Transition temperatures, when available from experiments or CALPHAD predictions, are also in good agreement: LTVC predicts transition temperatures slightly higher than the experimental values for binaries $(\sim 11 \%)$ and CALPHAD values for ternaries $(\sim 3 \%)$.

Cases in disagreement with experiments or CALPHAD, when found, are likely related to the neglect of vibrational formation entropy or to insufficient training data for cluster expansion, pointing to future directions for improvement of the method. The presented results identify potential stable solid solution candidates. Often, sluggish kinetics is the bottleneck in achieving equilibrium, so many transition temperatures might be quite difficult to characterize experimentally.

Analysis of 1110 quaternary and 130 quinary alloys show that $46 \%$ of them form solid solutions, suggesting that there are ample single phase HEAs yet to be discovered. Ab-initio modeling of hcp solid solution phases has not been performed yet, a future avenue of investigation for the model. Furthermore, the extension to nonequiatomic alloys still remains a daunting task without more information on concentration ranges.

It is proved that short range order effects are also crucial for correct solid solution-forming predictions: other methods, neglecting such effects on configurational entropy, are likely to significantly underestimate the transition temperature to a solid solution phase - possible erroneous prediction of solid solution-formation. Finally, previously suggested descriptors $[14,15,19,20]$ did not produce many of the solid solutions predicted here, casting doubts on the capability of such descriptors to serve as effective searching tools. The directions suggested in this article will facilitate the critical work of acquisition of new experimental data.

\section{ACKNOWLEDGMENTS}

We thank Ohad Levy, Donald Brenner, Jon-Paul Maria, Corey Oses, Matthias Scheffler, and Luca Ghiringhelli for various technical discussions. We acknowledge support by DOD-ONR (N00014-13-1-0635, N00014-11-1- 
0136, N00014-15-1-2863). S.C. acknowledges the Alexander von Humboldt Foundation for financial support. The consortium AFLOW.org acknowledges Duke University Center for Materials Genomics — for computational support.

\section{A. Binary alloys at equi-composition}

TABLE III: LTVC results for binary alloys. S.S. type of single solid solution phase ("none" = not found); $T_{\mathrm{c}}(\mathrm{K})$ transition temperature, as estimated by the method; $\tilde{T}_{\mathrm{c}}(\mathrm{K})$ approximated transition temperature, estimated according to Eq. (8); CV $(\mathrm{meV})$ cluster expansion cross validation score; S.S. Exp. type of single solid solution phase found experimentally ("none" = not found); $T_{\mathrm{c}}^{(\exp )}(\mathrm{K})$ experimental transition temperature; $T_{\mathrm{m}}^{(\exp )}(\mathrm{K})$ experimental melting temperature (solidus).

\begin{tabular}{|c|c|c|c|c|c|c|c|c|c|c|c|c|c|c|c|}
\hline & S.S. & $T_{\mathrm{c}}$ & $\tilde{T}_{\mathrm{c}}$ & $\mathrm{CV}$ & S.S. Exp. & $T_{\mathrm{c}}^{(\exp )}$ & $T_{\mathrm{m}}^{(\exp )}$ & & S.S. & $T_{\mathrm{c}}$ & $\tilde{T}_{\mathrm{c}}$ & $\mathrm{CV}$ & S.S. Exp. & $T_{\mathrm{c}}^{(\mathrm{e}}$ & $T_{\mathrm{m}}^{(\exp )}$ \\
\hline $\mathrm{AgAu}$ & fcc & 660 & 750 & 1 & fcc & & 1,300 & $\mathrm{AgCu}$ & none & 2,350 & 1,450 & 8 & none & & 1,070 \\
\hline $\mathrm{AgFe}$ & none & 6,590 & 5,400 & 22 & none & & 800 & $\mathrm{AgNi}$ & none & 5,620 & 3,480 & 21 & none & & 1,770 \\
\hline $\mathrm{AgPd}$ & fcc & 300 & 310 & 7 & fcc & & 540 & $\mathrm{AgPt}$ & fcc & 1,090 & 650 & 23 & fcc & & 1,840 \\
\hline $\mathrm{AgSi}$ & none & 5,270 & 5,390 & 36 & none & & 550 & $\mathrm{AgTi}$ & none & 2,790 & 3,050 & 45 & none & &, 720 \\
\hline $\mathrm{AlCu}$ & none & 1,130 & 1,480 & 21 & none & & 100 & AlHf & none & 2,400 & 3,790 & 47 & none & & 2,070 \\
\hline AlMn & one & 170 & 2,750 & 50 & none & & 470 & AlSi & none & 3,030 & 4,280 & 35 & none & & 1,320 \\
\hline $\mathrm{AlTa}$ & one & 960 & 2,540 & 48 & none & & 270 & AlTi & none & 3,480 & 2,850 & 50 & none & &, 770 \\
\hline AlW & none & 2,850 & 3,390 & 36 & none & & 920 & $\mathrm{AlZr}$ & none & 1,970 & 3,870 & 49 & none & &, 750 \\
\hline $\mathrm{AuCo}$ & none & 150 & 3,420 & 21 & none & & 470 & $\mathrm{uCu}$ & fcc & 670 & 680 & 19 & fcc & &, 180 \\
\hline $\mathrm{AuNi}$ & none & 3,050 & 1,820 & 21 & fcc & & 270 & $\mathrm{AuPd}$ & fcc & 490 & 630 & 6 & fcc & & 1,720 \\
\hline $\mathrm{AuPt}$ & fcc & 1,350 & 810 & 7 & fcc & 1,500 & 520 & $\mathrm{AuTi}$ & none & 2,880 & 2,820 & 47 & none & & 1,770 \\
\hline $\mathrm{AuV}$ & fcc & 310 & 1,850 & 30 & fcc & 0 & 650 & $\mathrm{CoCr}$ & none & 3,410 & 2,270 & 44 & bcc & & 1,670 \\
\hline $\mathrm{CoCu}$ & one & 2,820 & 1,910 & 10 & none & & 670 & CoIr & fcc & 010 & 630 & 17 & fcc & &, 770 \\
\hline CoMn & none & 620 & 1,600 & 14 & fcc & 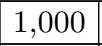 & 170 & $\mathrm{oMo}$ & none & 3,080 & 3,470 & 37 & none & & 820 \\
\hline $\mathrm{CoNi}$ & fcc & 300 & 320 & 6 & fcc & & 740 & $\mathrm{CoPd}$ & none & 1,540 & 00 & 15 & fcc & & 490 \\
\hline $\mathrm{CoPt}$ & fcc & 880 & 920 & 15 & fcc & 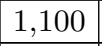 & 770 & CoRh & fcc & 1,400 & 1,010 & 8 & fcc & &, 670 \\
\hline CoW & none & 3,320 & 4,080 & 40 & none & &, 770 & $\mathrm{CrCu}$ & none & 6,310 & 4,260 & 17 & none & & 1,820 \\
\hline $\mathrm{CrFe}$ & none & 2,240 & 1,300 & 18 & bcc & $\pi 0$ & 820 & CrIr & none & 2,460 & 3,210 & 20 & none & & 2,270 \\
\hline CrMn & $\mathrm{bcc}$ & 950 & 970 & 8 & $\mathrm{bcc}$ & 0 & 670 & CrMo & bcc & 2,180 & 1,320 & 6 & bcc & 0 & 2,270 \\
\hline $\mathrm{CrNb}$ & none & 2,810 & 2,380 & 9 & none & & 920 & $\mathrm{CrNi}$ & fcc & 1,570 & 1,920 & 26 & fcc & 00 & 1,620 \\
\hline & fcc & 380 & 2,400 & 9 & fcc & & 0 & $\mathrm{hh}$ & one & 1,960 & 0 & 4 & 101 & & 1,870 \\
\hline $\mathrm{CrTa}$ & none & 540 & 3,010 & 13 & bcc & & 70 & $\Gamma \mathrm{i}$ & none & 30 & 2,770 & 38 & bcc & &, 680 \\
\hline $\mathrm{CrV}$ & bcc & 610 & 540 & 4 & bcc & & 070 & $\mathrm{CrW}$ & bcc & 2,190 & 1,360 & 8 & bcc & 60 & 2,570 \\
\hline $\mathrm{CuFe}$ & none & 2,910 & 2,300 & 28 & none & & 720 & CuIr & none & 2,270 & 1,740 & 26 & none & & 2,040 \\
\hline $\mathrm{CuNi}$ & fcc & 570 & 360 & 5 & fcc & & 520 & $\mathrm{CuPd}$ & fcc & 470 & 770 & 22 & fcc & U & 1,470 \\
\hline $\mathrm{CuPt}$ & fcc & 160 & 1,060 & 12 & fcc & 00 & 770 & $\mathrm{CuRh}$ & fcc & 1,170 & 880 & 19 & fcc & 30 & 1,570 \\
\hline $\mathrm{CuSi}$ & none & 3,600 & 3,770 & 17 & none & & 70 & IV & none & 2,480 & 2,250 & 40 & none & & 2,270 \\
\hline FeMn & fcc & 310 & 1,440 & 30 & fcc & & 20 & Mo & none & 2,880 & 2,020 & 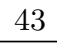 & none & & 2,070 \\
\hline $\mathrm{FeNb}$ & none & 2,390 & 2,770 & 27 & none & & 70 & $\mathrm{Ni}$ & none & 1,830 & 1,100 & 44 & bcc & & 1,710 \\
\hline $\mathrm{FePd}$ & fcc & 600 & 1,070 & 31 & fcc & & 580 & $\mathrm{HfNb}$ & bcc & 1,280 & 1,410 & 33 & $\mathrm{bcc}$ & 00 & 2,500 \\
\hline HfTa & bcc & 2,000 & 2,110 & 11 & bcc & 1,400 & 470 & HfTi & none & 2,540 & 2,190 & 21 & bcc & 400 & 1,970 \\
\hline HfV & none & 2,130 & 2,950 & 33 & none & & 770 & HfZr & none & 2,510 & 2,180 & 38 & bcc & 1,650 & 2,270 \\
\hline IrMo & none & 4,400 & 3,760 & 20 & none & & 2,470 & $\mathrm{IrNi}$ & fcc & 1,310 & 1,010 & 31 & fcc & 400 & 2,170 \\
\hline $\operatorname{IrPd}$ & none & 2,480 & 1,730 & 20 & fcc & & 970 & $\mathrm{IrPt}$ & fcc & 1,460 & 990 & 10 & fcc & 1,200 & 2,270 \\
\hline $\mathrm{IrRh}$ & fcc & 250 & 220 & 2 & fcc & & 2,570 & $\mathrm{IrRu}$ & fcc & 650 & 780 & 3 & fcc & & 2,670 \\
\hline $\mathrm{MnNb}$ & none & 4,810 & 3,380 & 30 & none & & 1,670 & $\mathrm{MnNi}$ & none & 2,380 & 1,980 & 22 & fcc & & 1,290 \\
\hline MnTa & none & 6,740 & 4,710 & 28 & none & & 1,670 & $\mathrm{MnV}$ & none & 3,800 & 2,970 & 14 & $\mathrm{bcc}$ & 1,070 & 1,820 \\
\hline $\mathrm{MoNb}$ & bcc & 1,040 & 900 & 4 & bcc & & 2,870 & MoNi & none & 2,220 & 2,860 & 35 & e & & 1,970 \\
\hline IoPd & fcc & 1,850 & 2,180 & 37 & fcc & 1,050 & 2,030 & MoRh & none & 3,090 & 2,710 & 25 & none & & 2,320 \\
\hline
\end{tabular}




\begin{tabular}{|c|c|c|c|c|c|c|c|c|c|c|c|c|c|c|c|}
\hline & S.S. & $T_{\mathrm{c}}$ & $\tilde{T}_{\mathrm{c}}$ & $\mathrm{CV}$ & S.S. Exp. & $T_{\mathrm{c}}^{(\exp )}$ & $T_{\mathrm{m}}^{(\exp )}$ & & S.S. & $T_{\mathrm{c}}$ & $\tilde{T}_{\mathrm{c}}$ & $\mathrm{CV}$ & S.S. Exp. & $T_{\mathrm{c}}^{(\exp )}$ & $T_{\mathrm{m}}^{(\exp )}$ \\
\hline MoRu & none & 2,610 & 2,910 & 14 & none & & 2,270 & MoTa & bcc & 1,850 & 1,560 & 5 & bcc & & 2,970 \\
\hline MoTi & bcc & 800 & 1,170 & 31 & $\mathrm{bcc}$ & 670 & 2,270 & $\mathrm{MoV}$ & bcc & 610 & 630 & 4 & bcc & & 2,470 \\
\hline MoW & bcc & 170 & 180 & 1 & $\mathrm{bcc}$ & & 3,220 & MoZr & none & 2,160 & 2,660 & 43 & none & & 1,820 \\
\hline $\mathrm{NbTa}$ & $\mathrm{bcc}$ & 140 & 130 & 1 & bcc & & 2,970 & $\mathrm{NbTi}$ & $\mathrm{bcc}$ & 650 & 760 & 6 & bcc & 670 & 2,170 \\
\hline $\mathrm{NbV}$ & bcc & 1,480 & 1,630 & 3 & bcc & & 2,220 & $\mathrm{NbW}$ & bcc & 330 & 400 & 4 & bcc & & 2,920 \\
\hline $\mathrm{NbZr}$ & $\mathrm{bcc}$ & 1,190 & 1,420 & 11 & $\mathrm{bcc}$ & 1,200 & 2,070 & $\mathrm{NiPd}$ & $\mathrm{bcc}$ & 1,120 & 700 & 31 & bcc & 570 & 1,510 \\
\hline $\mathrm{NiPt}$ & fcc & 1,110 & 880 & 19 & fcc & 950 & 1,720 & $\mathrm{NiRh}$ & fcc & 940 & 660 & 13 & fcc & & 1,820 \\
\hline $\mathrm{NiRu}$ & fcc & 1,720 & 1,910 & 20 & none & & 1,820 & $\mathrm{NiV}$ & none & 1,980 & 2,330 & 43 & none & & 1,470 \\
\hline $\mathrm{NiW}$ & none & 3,110 & 3,690 & 49 & none & & 2,670 & $\mathrm{PdPt}$ & fcc & 310 & 270 & 2 & fcc & & 1,840 \\
\hline $\mathrm{PdRh}$ & fcc & 1,610 & 1,060 & 5 & fcc & 1,120 & 1,920 & $\mathrm{PdRu}$ & none & 2,260 & 2,590 & 16 & none & & 1,850 \\
\hline $\mathrm{PdW}$ & none & 2,370 & 2,890 & 40 & none & & 2,085 & PtRh & fcc & 210 & 240 & 3 & fcc & & 2,170 \\
\hline $\mathrm{PtRu}$ & fcc & 1,560 & 1,480 & 10 & fcc & & 2,370 & PtTi & none & 3,120 & 4,700 & 48 & none & & 2,100 \\
\hline $\mathrm{PtW}$ & none & 2,560 & 2,970 & 43 & none & & 2,500 & RhRu & fcc & 870 & 900 & 7 & fcc & & 2,370 \\
\hline $\mathrm{RhV}$ & fcc & 1,320 & 2,650 & 46 & none & & 1,820 & RhW & none & 2,870 & 2,900 & 28 & none & & 2,520 \\
\hline RuW & none & 2,740 & 3,140 & 17 & none & & 2,470 & TaTi & bcc & 1,110 & 1,150 & 3 & bcc & & 2,470 \\
\hline $\mathrm{TaV}$ & bcc & 2,260 & 2,360 & 3 & bcc & 1,400 & 2,370 & TaW & bcc & 690 & 640 & 13 & bcc & & 3,450 \\
\hline TaZr & bcc & 2,090 & 2,070 & 9 & bcc & 2,000 & 2,120 & $\mathrm{TiV}$ & bcc & 1,210 & 1,390 & 10 & $\mathrm{bcc}$ & 1,120 & 1,880 \\
\hline TiW & bcc & 710 & 890 & 33 & $\mathrm{bcc}$ & 1,000 & 2,220 & TiZr & bcc & 1,340 & 1,600 & 38 & bcc & 880 & 1,820 \\
\hline VW & bcc & 200 & 360 & 11 & bcc & & 2,570 & VZr & none & 3,470 & 3,050 & 29 & none & & 1,570 \\
\hline WZr & none & 3,950 & 4,170 & 25 & none & & 3,070 & & & & & & & & \\
\hline
\end{tabular}

\section{B. Ternary alloys at equi-composition}

TABLE IV: LTVC results for ternary alloys. S.S. type of single solid solution phase ("none" = not found); $T_{\mathrm{c}}(\mathrm{K})$ transition temperature, as estimated by the method; $\tilde{T}_{\mathrm{c}}(\mathrm{K})$ approximated transition temperature, estimated according to Eq. (8); CV $(\mathrm{meV})$ cluster expansion cross validation score; S.S. ${ }^{(*)}$ type of single solid solution phase using CALPHAD ("none" = not found); $T_{\mathrm{c}}^{(*)}(\mathrm{K})$ transition temperature, as estimated by CALPHAD; $T_{\mathrm{m}}^{(*)}(\mathrm{K})$ melting temperature (solidus), as estimated by CALPHAD; $n^{(\star)}$ number of binary alloys (3 possible for each ternary) found in the SSOL5 database of Thermo-Calc.

\begin{tabular}{|c|c|c|c|c|c|c|c|c|c|c|c|c|c|c|c|c|c|}
\hline & \begin{tabular}{|l|} 
S.S. \\
\end{tabular} & $T_{\mathrm{c}}$ & $\tilde{T}_{\mathrm{c}}$ & $\mathrm{CV}$ & S.S. ${ }^{(*)}$ & $T_{\mathrm{c}}^{(\star)}$ & $T_{\mathrm{m}}^{(\star)}$ & $n^{(\star)}$ & & S.S. & $T_{\mathrm{c}}$ & $\tilde{T}_{\mathrm{C}}$ & $\mathrm{CV}$ & S.S. ${ }^{(\star)}$ & $T_{\mathrm{c}}^{(\star)}$ & \begin{tabular}{|l|}
$T_{\mathrm{m}}^{(*)}$ \\
\end{tabular} & $n^{(\star)}$ \\
\hline $\mathrm{gAlAu}$ & none & \begin{tabular}{|l|}
2,670 \\
\end{tabular} & 2,530 & 27 & fcc & \begin{tabular}{|l|}
830 \\
\end{tabular} & \begin{tabular}{|l|}
970 \\
\end{tabular} & 3 & gAlFe & none & 7,000 & 7,000 & 38 & none & & 1,050 & 3 \\
\hline AgAlNi & none & 6 & 3,690 & 37 & none & & 1,220 & 3 & gAlV & none & 5,160 & 2,650 & 45 & ione & & 1,040 & 3 \\
\hline $\mathrm{sAu}$ & ne & 780 & 820 & 36 & none & & 900 & 2 & $\mathrm{AsCu}$ & one & 1,920 & 1,780 & 27 & he & & 800 & 1 \\
\hline $\mathrm{sPd}$ & ne & 660 & 1,620 & 37 & none & & 1,010 & 1 & $\mathrm{gAuCo}$ & one & 4,880 & 2,920 & 35 & none & & 1,310 & 2 \\
\hline${ }_{1} \mathrm{Cu}$ & ne & 320 & 720 & 10 & fcc & 06 & 1,080 & 3 & $\mathrm{uFe}$ & one & 6,490 & 2,680 & 41 & none & & 280 & 2 \\
\hline $\mathrm{AMo}$ & none & 4,200 & 3,870 & 39 & none & & 1,280 & 2 & gAuNi & none & 4,840 & 1,850 & 15 & none & & 1,420 & 3 \\
\hline $\mathrm{uPd}$ & fcc & 250 & 390 & 6 & fcc & 273 & 1,540 & 3 & $\mathrm{gAuPt}$ & fcc & 1,110 & 560 & 7 & fcc & 1,070 & 1,450 & 2 \\
\hline $\mathrm{AgAuRh}$ & none & 4,860 & 1,970 & 14 & none & & 2,170 & 3 & $\mathrm{AgAuSi}$ & none & 3,100 & 2,450 & 39 & none & & 920 & 3 \\
\hline AgAuTi & none & 2,350 & 1,830 & 38 & none & & 1,230 & 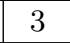 & $\mathrm{uV}$ & none & 4,630 & 2,340 & 38 & none & & 1,290 & 2 \\
\hline $\mathrm{AgCoCu}$ & none & 5,440 & 2,570 & 22 & none & & 1,250 & 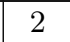 & $\mathrm{oPd}$ & none & 5,230 & 2,040 & 21 & fcc & 650 & 1,500 & 2 \\
\hline $\mathrm{AgCoPt}$ & none & 5,020 & 2,090 & 30 & none & & 1,370 & -1 & $\mathrm{oRh}$ & none & 6,390 & 2,670 & 24 & none & & $\mid 1,370$ & 1 \\
\hline $\mathrm{uFe}$ & none & 5,090 & 2,560 & 28 & none & & 1,050 & 3 & uIr & none & 4,940 & 2,440 & 32 & none & & 1,050 & 3 \\
\hline $\mathrm{uMn}$ & none & 5,260 & 2,200 & 48 & none & & 1,060 & 3 & $\mathrm{uNi}$ & none & 3,730 & 1,520 & 14 & none & & 1,600 & 3 \\
\hline $\mathrm{uPd}$ & one & 1,400 & 800 & 9 & none & & 1,340 & 3 & $\mathrm{uPt}$ & none & 1,970 & 1,160 & 12 & none & & 1,190 & 2 \\
\hline $\mathrm{Rh}$ & ne & 3,290 & 1,540 & 17 & none & & 1,060 & 3 & $\mathrm{Si}$ & none & 2,570 & 2,430 & 22 & none & & 1,040 & 3 \\
\hline & none & 2,740 & 1,910 & 41 & none & & 1,150 & 3 & $\mathrm{uV}$ & none & 5,000 & 2,610 & 41 & none & & 1,110 & 3 \\
\hline & none & 1,130 & 1,660 & 48 & none & & 1,040 & 3 & & none & 6,920 & 2,830 & 25 & none & & 1,230 & 3 \\
\hline $\mathrm{AgFePd}$ & none & 4,320 & 1,990 & 43 & none & & 1,320 & 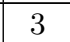 & AgHfZr & fcc & 1,210 & 1,150 & 44 & $\mathrm{bcc}$ & 1,360 & 1,710 & 2 \\
\hline $\mathrm{AgIrNi}$ & none & 6,630 & 2,850 & 30 & none & & 1,230 & 3 & AgIrPd & none & 6,110 & 2,560 & 35 & none & & 1,530 & 3 \\
\hline $\mathrm{AgIrPt}$ & none & 4,960 & 2,270 & 29 & none & & 1,390 & 3 & $\mathrm{Rh}$ & none & 6,770 & 2,640 & 25 & none & & 1,230 & 3 \\
\hline $\mathrm{AgNiPd}$ & none & 3,730 & 1,430 & 14 & none & & 1,430 & 3 & $\mathrm{AgNiPt}$ & none & 3,270 & 1,530 & 26 & none & & 1,310 & 2 \\
\hline
\end{tabular}




\begin{tabular}{|c|c|c|c|c|c|c|c|c|c|c|c|c|c|c|c|c|c|}
\hline & S.S. & $T_{\mathrm{c}}$ & $\tilde{T}_{\mathrm{c}}$ & CV & S.S..$^{(*)}$ & $T_{\mathrm{c}}^{(\star)}$ & $T_{\mathrm{m}}^{(\star)}$ & 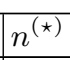 & & S.S. & $T_{\mathrm{c}}$ & $\tilde{T}_{\mathrm{c}}$ & $\mathrm{CV}$ & S.S. ${ }^{(\star)}$ & $T_{\mathrm{c}}^{(\star)}$ & $T_{\mathrm{m}}^{(\star)}$ & $n^{(\star)}$ \\
\hline $\mathrm{Rh}$ & none & 4,900 & $\mid 2,110$ & 20 & none & & 1,230 & ( & 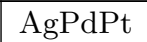 & fcc & 480 & 440 & 8 & fcc & 1,170 & 1,610 & $\tau^{2}$ \\
\hline $\mathrm{dRh}$ & one & 850 & 1,580 & 12 & none & & 530 & 3 & $\mathrm{gPdV}$ & none & 4,530 & 2,260 & 41 & none & & 1,490 & 2 \\
\hline thh & none & 3,090 & 1,340 & 14 & none & & 1,300 & 3 & gPtRu & fcc & 790 & 2,510 & 35 & none & & 1,260 & 3 \\
\hline AgPtTi & none & 5,440 & 3,410 & 44 & none & & 1,230 & 3 & $\mathrm{AgPtV}$ & none & 6,980 & 3,770 & 48 & none & & 1,360 & 2 \\
\hline AgTiV & none & 4,010 & 2,530 & 46 & none & & 1,230 & 3 & gTiZr & none & 3,840 & 1,710 & 45 & bcc & 1,120 & 1,540 & 3 \\
\hline $\mathrm{AlAuCu}$ & none & 1,400 & 1,730 & 41 & fcc & 750 & 960 & 3 & $1 \mathrm{AuNi}$ & none & 3,810 & 2,230 & 47 & none & & 1,050 & 3 \\
\hline AlAuSi & one & 3,340 & 3,540 & 46 & none & & 780 & 3 & $\mathrm{CoCr}$ & none & 6,620 & 3,660 & 47 & none & & 1,520 & 3 \\
\hline $\mathrm{AlCoCu}$ & one & 2,400 & 2,000 & 42 & none & & 1,320 & 3 & $\mathrm{lCoNi}$ & one & 2,400 & 3,290 & 48 & none & & 1,440 & 3 \\
\hline $\mathrm{AlCrCu}$ & ne & 3,670 & 2,310 & 50 & none & & 1,200 & 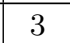 & $\mathrm{rMn}$ & one & 1,610 & 1,480 & 46 & bcc & 273 & 1,590 & 3 \\
\hline $\mathrm{AlCrV}$ & none & 2,160 & 1,130 & 48 & $\mathrm{bcc}$ & 273 & $\mid 1,970$ & 3 & & none & 2,380 & 1,580 & 43 & none & & 1,600 & 3 \\
\hline $\mathrm{AlCuFe}$ & none & 140 & 1,960 & 47 & none & & 1,310 & 3 & $\mathrm{Mn}$ & one & 2,690 & 1,630 & 50 & bcc & 880 & 1,310 & 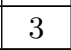 \\
\hline & one & 360 & 2,180 & 4 & one & & 1,340 & 3 & & none & 2,330 & 2,380 & 18 & one & & 840 & 3 \\
\hline & one & 000 & 1,420 & 49 & none & & 1,570 & 3 & & none & 2,390 & 3,740 & 49 & none & & 930 & 2 \\
\hline & none & 530 & 1,750 & 39 & none & & 1,570 & 2 & & fcc & 1,000 & 1,600 & 34 & bcc & 0 & 1,730 & 2 \\
\hline & none & 2,760 & 2,230 & 48 & none & & 1,350 & 2 & $\mathrm{nSi}$ & none & 2,720 & 3,300 & 47 & none & & 1,110 & 3 \\
\hline & none & 3,190 & 2,150 & 45 & none & & 1,580 & 3 & & none & 2,820 & 1,820 & 39 & bcc & 1,010 & 2,000 & 3 \\
\hline AlTiZr & fcc & 1,630 & 1,830 & 49 & $\mathrm{bcc}$ & 1,420 & 1,650 & 3 & & none & 2,490 & 1,180 & 49 & $\mathrm{bcc}$ & 2,060 & 2,080 & 3 \\
\hline $\mathrm{AsAuCu}$ & none & 1,750 & 1,710 & 34 & none & & 920 & 2 & $\mathrm{uPd}$ & none & 2,620 & 2,080 & 45 & none & & 1,010 & 2 \\
\hline $\mathrm{AsCuPd}$ & none & 1,660 & 1,560 & 43 & none & & 1,020 & 1 & $\mathrm{CoCu}$ & none & 3,970 & 1,800 & 36 & none & 720 & 1,640 & 3 \\
\hline & nne & 6,600 & 2,520 & 29 & fcc & 1 & 1,880 & 1 & $\mathrm{OMo}$ & one & 5,050 & 2,930 & 39 & one & & 1,500 & 2 \\
\hline & one & 3,060 & 740 & 43 & none & & 1,300 & 3 & & one & 3,710 & 1,600 & 15 & fcc & 50 & 1,450 & 3 \\
\hline & one & \begin{tabular}{|l|}
3,610 \\
\end{tabular} & 1,680 & 16 & none & & $\mid 1,520$ & 2 & $\mathrm{Rh}$ & one & 4,040 & 1,760 & 17 & ione & & 1,590 & 2 \\
\hline & none & 3,630 & 1,940 & 38 & one & 2 & 1,480 & 2 & & one & 3,850 & 1,620 & 38 & none & & 1,220 & 2 \\
\hline & none & 5,170 & 2,290 & 29 & none & & 1,270 & 2 & & none & 1,980 & 900 & 16 & fcc & 1,120 & 1,250 & 3 \\
\hline & bcc & 970 & 700 & 2 & fcc & & 1,440 & 3 & & fcc & 1,330 & 880 & 1 & fcc & 273 & 1,440 & 1 \\
\hline & one & 2,620 & 1,190 & 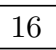 & none & & 1,190 & 3 & & none & 2,910 & 1,660 & 39 & none & & 750 & 3 \\
\hline $\mathrm{iTi}$ & ne & 1,330 & 1,570 & 41 & none & & 1,210 & 3 & & fcc & 1,240 & 1,340 & 34 & none & & 1,570 & 2 \\
\hline & none & 3,280 & 3,690 & 48 & none & & 1,230 & 1 & & none & 3,050 & 1,560 & 43 & fcc & 380 & 1,420 & 2 \\
\hline $\mathrm{ePd}$ & fcc & 1,060 & 960 & 46 & fcc & 710 & 1,600 & 2 & & fcc & 270 & 1,420 & 48 & none & & 1,270 & 3 \\
\hline $\mathrm{AuIrNi}$ & none & 5,090 & 2,360 & 30 & fcc & 800 & 1,690 & 2 & & none & 5,930 & 2,620 & 25 & fcc & 1,290 & 1,960 & 2 \\
\hline $\mathrm{rPt}$ & none & 4,850 & 2,040 & 2 & fcc & 1,05 & 1,850 & 1 & & none & 5,960 & 2,280 & 23 & none & & 1,830 & 2 \\
\hline & none & 4,100 & 2,490 & 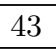 & none & & 1,290 & 2 & & one & 1,750 & 1,910 & 38 & none & & 1,680 & 2 \\
\hline & none & 5,400 & 2,630 & 35 & none & & 1,670 & 1 & & none & 2,610 & 1,100 & 15 & fcc & 310 & $\mid 1,440$ & 3 \\
\hline & one & 2,350 & 10 & 2 & fcc & & 1,530 & 1 & $2 \mathrm{~h}$ & none & 2,880 & 1,380 & 17 & none & & 1,250 & 2 \\
\hline & none & 2,730 & 690 & & fcc & 110 & 1,250 & 2 & $\mathrm{t}$ & fcc & 1,200 & 650 & & fcc & 273 & 1,790 & 1 \\
\hline & none & 3,340 & 1,450 & 1 & one & & 1,700 & 3 & & none & 1,740 & 1,910 & 43 & fcc & 1,580 & 1,630 & 2 \\
\hline & fcc & 750 & 000 & 3 & none & & 1,540 & 1 & $\mathrm{~W}$ & none & 2,230 & 2,510 & 50 & none & & 1,770 & 1 \\
\hline $\mathrm{Rh}$ & one & 2,590 & 1,110 & 1 & none & & 1,550 & 2 & & none & 4,320 & 2,090 & 32 & none & & 1,510 & 2 \\
\hline $\mathrm{tTi}$ & none & 1,970 & 2,820 & 46 & none & & 1,670 & 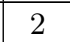 & & none & 3,650 & 2,240 & 42 & none & & 1,400 & 1 \\
\hline AuRhW & none & 6,630 & 3,170 & 46 & none & & 1,790 & - & & fcc & 1,180 & 1,430 & 48 & none & & 1,710 & 2 \\
\hline$\Gamma \mathrm{iV}$ & fcc & 1,540 & 1,870 & 44 & none & & 1,670 & - & & fcc & 410 & 1,540 & 47 & fcc & 1,210 & 1,390 & 3 \\
\hline $\mathrm{CoCrCu}$ & none & 3,920 & 2,090 & 35 & none & & 1,590 & 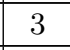 & $\mathrm{CrIr}$ & fcc & 1,660 & 1,780 & 26 & fcc & 970 & 1,970 & 1 \\
\hline CoCrMn & none & 3,080 & 1,550 & 41 & $\mathrm{bcc}$ & 1,320 & 1,510 & 3 & $\mathrm{CrNb}$ & none & 3,370 & 2,170 & 46 & none & & 1,430 & 3 \\
\hline $\mathrm{rNi}$ & fcc & 1,350 & 890 & 2 & fcc & 9 & 1,670 & 3 & $\mathrm{rPd}$ & none & 3,210 & 2,040 & 39 & fcc & 90 & 1,540 & 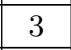 \\
\hline & ne & 2,030 & 1,390 & 3 & fcc & 89 & 820 & 3 & & fcc & 610 & 1,330 & 29 & fcc & 1,110 & 1,820 & 1 \\
\hline & none & 3,330 & 930 & 4 & none & & 1,680 & 3 & & none & 2,640 & 1,260 & 28 & none & & 1,750 & 2 \\
\hline & none & 2,410 & 1,520 & 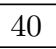 & none & & 1,180 & 3 & & bcc & 1,400 & 1,120 & 35 & none & & 1,600 & 3 \\
\hline $\mathrm{iPd}$ & one & 3,020 & 1,610 & 21 & fcc & 1,420 & 1,460 & 3 & & none & 2,050 & 1,310 & 22 & none & & 1,460 & 2 \\
\hline $\mathrm{uRh}$ & none & 1,720 & 1,020 & 14 & none & & 1,670 & 2 & $\mathrm{uV}$ & none & 3,430 & 1,970 & 41 & none & & 2,090 & 3 \\
\hline $\mathrm{emn}$ & one & 2,770 & 1,300 & 49 & fcc & & 1,550 & 3 & $\mathrm{Mn}$ & none & 2,110 & 1,540 & 19 & fcc & 273 & 1,820 & 1 \\
\hline CoIrMo & none & 2,860 & 2,160 & 29 & fcc & 180 & 2,110 & 1 & & fcc & 1,040 & 730 & 22 & fcc & 740 & 2,030 & 2 \\
\hline $\mathrm{rPd}$ & none & 1,850 & 1,080 & 20 & fcc & 1,550 & 1,650 & 2 & & fcc & 1,670 & 950 & 19 & fcc & 1,280 & 1,910 & 2 \\
\hline CoIrRh & fcc & 1,250 & 520 & 13 & fcc & 690 & 2,150 & 1 & CoIrV & none & 3,520 & 2,090 & 47 & fcc & 1,290 & 1,650 & 1 \\
\hline
\end{tabular}




\begin{tabular}{|c|c|c|c|c|c|c|c|c|c|c|c|c|c|c|c|c|c|}
\hline & S.S. & $T_{\mathrm{c}}$ & $\tilde{T}_{\mathrm{c}}$ & $\mathrm{CV}$ & 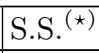 & $T_{\mathrm{c}}^{(\star)}$ & $T_{\mathrm{m}}^{(\star)}$ & 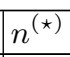 & & S.S. & $T_{\mathrm{c}}$ & $\tilde{T}_{\mathrm{c}}$ & $6 \mathrm{v}$ & .S..$^{(\star)}$ & $T_{\mathrm{c}}^{(\star)}$ & $T_{\mathrm{m}}^{(\star)}$ & $n^{(\star)}$ \\
\hline $\mathrm{Co}$ & none & 3,110 & 2,350 & 35 & none & & 2,070 & 1 & $\mathrm{MnNi}$ & none & 2,100 & 1,070 & 30 & fcc & 600 & 1,440 & 2 \\
\hline $\mathrm{InRh}$ & none & 020 & 1,920 & 29 & fcc & 273 & 710 & 1 & $\mathrm{MoNi}$ & fcc & 130 & 1,560 & 41 & none & & 1,600 & 3 \\
\hline CoMoPt & $\mathrm{fcc}$ & 250 & 1,780 & 45 & none & & 1,740 & 2 & $\mathrm{oMoRh}$ & none & 2,140 & 1,980 & 38 & fcc & 1,370 & 1,920 & 1 \\
\hline CoNiPd & fcc & 1,430 & 710 & 28 & fcc & 273 & 1,520 & 3 & $\mathrm{CoNiPt}$ & fcc & 800 & 590 & 22 & fcc & 620 & 1,780 & 2 \\
\hline $\mathrm{CoNiRh}$ & fcc & 1,340 & 630 & 19 & fcc & 740 & 1,900 & 1 & $\mathrm{CoNiV}$ & none & 2,750 & 1,450 & 38 & fcc & 750 & 490 & 3 \\
\hline $\mathrm{CoPdPt}$ & fcc & 1,030 & 670 & 14 & fcc & 670 & 1,650 & 2 & CoPdRh & fcc & 1,520 & 940 & 23 & fcc & 1,080 & 1,670 & 2 \\
\hline CoPdV & none & 2,430 & 1,780 & 41 & none & & 1,400 & 2 & CoPdW & none & 2,780 & 1,800 & 48 & none & & 1,550 & 2 \\
\hline CoPtRh & fcc & 060 & 650 & 15 & fcc & 610 & 1,960 & 2 & & none & 2,200 & 1,920 & 50 & fcc & 1,310 & 1,500 & 2 \\
\hline CoRhV & none & 3,120 & 1,910 & 42 & fcc & 1,290 & 1,570 & 1 & & one & 3,310 & 2,220 & 35 & none & & 1,920 & -1 \\
\hline $\mathrm{uFe}$ & none & 4,540 & 2,320 & 40 & none & & 1,350 & 3 & पo & none & 5,520 & 2,630 & 50 & one & & 1,340 & 3 \\
\hline & none & 2,770 & 1,750 & 6 & none & & 1,390 & 3 & & none & 3,050 & 2,210 & 41 & one & & 1,440 & 3 \\
\hline & none & 2,220 & 1,890 & 1 & none & & 1,360 & 2 & & none & 3,260 & 1,700 & 46 & one & & 1,390 & 2 \\
\hline & none & 4,980 & 2,220 & 8 & none & & 1,570 & 3 & & none & 6,620 & 3,060 & 45 & ione & & 1,770 & 2 \\
\hline & fcc & 1,860 & 550 & 3 & fcc & 050 & 1,970 & 1 & & $\mathrm{bcc}$ & 1,040 & 800 & 25 & bcc & 1,380 & 1,690 & 3 \\
\hline $\mathrm{CrF}$ & none & 2,300 & 350 & 26 & none & 1,400 & 2,030 & 3 & & none & 2,460 & 1,900 & 29 & none & & 1,450 & 3 \\
\hline $\mathrm{Cr}$ & none & 2,860 & 1,530 & 48 & fcc & 1,230 & 1,660 & 3 & & none & 2,170 & 1,500 & 42 & fcc & 1,220 & 1,780 & 1 \\
\hline $\mathrm{CrFeTa}$ & none & 3,740 & 2,610 & 32 & none & & 1,660 & 3 & & none & 4,920 & 2,940 & 41 & none & & 1,470 & 3 \\
\hline $\mathrm{CrFeV}$ & bcc & 1,830 & 1,070 & 2 & $\mathrm{bcc}$ & 1,160 & 1,900 & 3 & & none & 2,300 & 1,440 & 37 & none & & 1,930 & 3 \\
\hline CrHfNb & none & 3,130 & 2,300 & 3 & $\mathrm{bcc}$ & 1,410 & 2,040 & 2 & & $\mathrm{bcc}$ & 2,060 & 2,530 & 31 & $\mathrm{bcc}$ & 1,660 & 2,220 & 2 \\
\hline & none & 2,010 & 2,290 & 40 & $\mathrm{bcc}$ & 1, & 1,900 & 2 & & none & 2,950 & 3,270 & 32 & one & & ,190 & 2 \\
\hline & none & 3,320 & 1,930 & 43 & fcc & 1,720 & 1,770 & 1 & & fcc & 1,620 & 1,200 & 29 & fcc & 930 & 1,930 & 2 \\
\hline & none & 3,490 & 2,340 & 45 & fcc & $\mid 1,470$ & 1,780 & 2 & & none & 2,270 & 1,820 & 32 & none & & $2,010 \mid$ & 2 \\
\hline & fcc & 100 & 1,180 & 2 & fcc & 690 & 1,960 & 1 & & none & 2,150 & 1,530 & $=$ & $\mathrm{bcc}$ & 690 & 1,830 & 3 \\
\hline & none & 3,690 & 2,100 & & bcc & 1,50 & 1,780 & 2 & & none & 3,170 & 1,410 & 4 & one & & 400 & 2 \\
\hline & none & 3,100 & 2,030 & 17 & none & & 1,700 & 1 & & none & 4,900 & 2,590 & $2:$ & bcc & 1,790 & 1,880 & 2 \\
\hline & ne & 2,970 & 2,150 & & none & & 1,740 & 3 & & none & 2,630 & 1,380 & $1:$ & $\mathrm{cc}$ & 640 & 1,860 & 3 \\
\hline & none & 2,230 & 1,660 & 18 & none & & 2,170 & 3 & & none & 3,150 & 1,380 & & $\mathrm{bcc}$ & 1,680 & 2,090 & 3 \\
\hline & bcc & 1,170 & 1,040 & 2 & bcc & 2,030 & 2,410 & 1 & & none & 2,780 & 1,800 & & $\mathrm{bcc}$ & 2,010 & 2,030 & 3 \\
\hline & $\mathrm{bcc}$ & 1,230 & 1,050 & 1 & $\mathrm{bcc}$ & 1,930 & 2,150 & 1 & & $\mathrm{bcc}$ & 1,930 & 1,580 & 27 & $\mathrm{bcc}$ & 1,390 & 1,940 & 3 \\
\hline & bcc & 1,260 & 790 & 4 & $\mathrm{bcc}$ & 1,280 & 2,310 & 3 & & $\mathrm{bcc}$ & 2,260 & 820 & S & $\mathrm{bcc}$ & 1,410 & 2,510 & 3 \\
\hline CrMoZr & none & 3,850 & 2,610 & 30 & none & & 1,980 & 3 & & none & 2,250 & 1,370 & & none & & 2,000 & 3 \\
\hline & none & 1,980 & 1,460 & 19 & none & & 1,690 & 3 & & none & 2,520 & 1,210 & 7 & bcc & 290 & 1,950 & 3 \\
\hline & one & 2,930 & 1,260 & 8 & $\mathrm{bcc}$ & 0 & 1,990 & 3 & & none & 3,540 & 2,200 & 7 & none & & $|1,640|$ & 3 \\
\hline & one & 20 & 50 & & fo & & 1,540 & 3 & & fcc & 1,560 & 1,320 & & fcc & 700 & 1,790 & 2 \\
\hline & fcc & 430 & 1,130 & & fcc & 70 & 1,810 & 1 & & none & 2,390 & 1,620 & 40 & none & & 1,790 & 2 \\
\hline & ne & 2,550 & 1,750 & 4 & fo & 0 & 1,740 & 2 & & none & 2,120 & 1,360 & & fcc & ,960 & 2,040 & 2 \\
\hline & ne & 4,530 & 2,710 & 2 & $\mathrm{bcc}$ & 110 & 2,310 & 1 & & $\mathrm{bcc}$ & 1,010 & 1,000 & 2 & $\mathrm{bcc}$ & 850 & 2,600 & 1 \\
\hline & bcc & 420 & 1,640 & 2 & none & & 1,760 & 3 & & $\mathrm{bcc}$ & 1,700 & 1,790 & & $\mathrm{bcc}$ & 800 & 2,120 & 3 \\
\hline & bcc & 2,320 & 1,690 & 10 & $\mathrm{bcc}$ & 2,050 & 2,390 & 3 & & none & 2,980 & 2,670 & 28 & none & & 1,630 & 3 \\
\hline & bcc & 1,030 & 990 & 17 & $\mathrm{bcc}$ & 1,780 & 2,250 & 1 & & $\mathrm{bcc}$ & 1,150 & 1,040 & 15 & $\mathrm{bcc}$ & 060 & 1,640 & 3 \\
\hline CrTiW & $\mathrm{bcc}$ & 1,470 & 1,540 & 18 & none & & 1,930 & 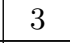 & & none & 3,970 & 2,280 & 49 & one & & 1,570 & 3 \\
\hline CrVW & bcc & 1,220 & 760 & 7 & $\mathrm{bcc}$ & 1,01 & 2,330 & 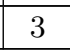 & & none & 3,580 & 3,080 & 29 & none & & 1,750 & 3 \\
\hline CuFeMn & none & 2,830 & 1,700 & 47 & none & & 1,180 & 5 & $\mathrm{Ni}$ & none & 1,670 & 960 & 19 & fcc & 1,340 & 1,510 & 3 \\
\hline $\mathrm{Cu}$ & fcc & 1,360 & 930 & 3 & none & & 430 & 3 & $\mathrm{Rh}$ & none & 2,340 & 1,070 & 44 & ione & & 1,420 & 2 \\
\hline $\mathrm{FeV}$ & none & 3,750 & 1,850 & 3 & none & & 1,470 & 3 & & fcc & 730 & 1,170 & 42 & one & & 1,050 & 3 \\
\hline $\mathrm{Cu}$ & fcc & 560 & 1,210 & 48 & none & & 990 & 3 & & none & 2,610 & 2,020 & 41 & one & & 210 & 2 \\
\hline & fcc & 1,150 & 970 & 2 & none & & 1,530 & 3 & & none & 3,260 & 1,650 & 2 & ione & & 1,530 & 3 \\
\hline & none & 2,800 & 1,530 & 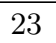 & none & & 1,610 & 2 & & none & 1,760 & 860 & 2 & none & & 1,520 & 3 \\
\hline $\mathrm{nNi}$ & none & 2,290 & 1,400 & 3 & fcc & 1,080 & 1,270 & 2 & & none & 1,680 & 920 & 1 & fcc & 10 &, 500 & 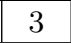 \\
\hline $\mathrm{CuNiPt}$ & fcc & 940 & 750 & $1:$ & fcc & 273 & 1,690 & 1 & $\mathrm{Rh}$ & fcc & 1,100 & 610 & 13 & fcc & 1,180 & 1,650 & 2 \\
\hline $\mathrm{CuNiSi}$ & none & 3,310 & 2,430 & 41 & none & & 1,100 & 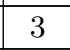 & $\mathrm{iV}$ & none & 2,400 & 1,440 & 38 & none & & 1,490 & 3 \\
\hline $\mathrm{CuPdPt}$ & fcc & 650 & 460 & 9 & fcc & $v^{2}$ & 1,670 & 1 & $\mathrm{Rh}$ & none & 2,230 & 1,140 & 13 & fcc & 1,510 & 1,610 & 3 \\
\hline $\mathrm{CuPdTi}$ & none & 1,400 & 1,750 & 45 & fcc & 920 & 1,350 & 2 & $\mathrm{CuPdV}$ & fcc & 1,310 & 1,150 & 38 & none & & 1,470 & 2 \\
\hline
\end{tabular}




\begin{tabular}{|c|c|c|c|c|c|c|c|c|c|c|c|c|c|c|c|c|c|}
\hline & S. & $T_{\mathrm{c}}$ & $\tilde{T}_{\mathrm{c}}$ & CV & S.S. ${ }^{(*)}$ & $T_{\mathrm{c}}^{(\star)}$ & $T_{\mathrm{m}}^{(\star)}$ & 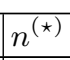 & & .N. & $I_{\mathrm{C}}$ & $\tilde{T}_{\mathrm{c}}$ & $\mathrm{CV}$ & S.S. & $T_{\mathrm{c}}^{(\star)}$ & $T_{\mathrm{m}}^{(\star)}$ & $n^{(\star)}$ \\
\hline $\mathrm{Rh}$ & $\overline{f c c}$ & 450 & 930 & 14 & fcc & 120 & 1,800 & ( & $u$ & one & 2,790 & 1,520 & 31 & fcc & 1,680 & 1,870 & 1 \\
\hline uPtTi & nne & 550 & 2,530 & 46 & one & & 1,310 & 2 & & none & 3,060 & 2,220 & 45 & none & & 1,600 & 1 \\
\hline $\mathrm{hV}$ & one & 3,410 & 1,920 & 44 & none & & 1,570 & 2 & & fcc & 510 & 1,710 & 46 & bcc & 140 & 1,530 & - \\
\hline $\mathrm{CuTaV}$ & \begin{tabular}{|l|} 
none \\
\end{tabular} & 2,740 & \begin{tabular}{|l|}
2,140 \\
\end{tabular} & 49 & none & & \begin{tabular}{|l|}
1,820 \\
\end{tabular} & 2 & $\mathrm{uTiV}$ & none & 1,560 & 1,750 & 42 & none & & 1,190 & 3 \\
\hline CuTiZr & none & 2,230 & 1,440 & 39 & none & & 980 & 3 & $\mathrm{iVW}$ & none & 5,530 & 2,350 & 49 & bcc & 1,870 & 970 & 2 \\
\hline FeIrMn & none & 2,340 & 1,520 & 34 & fcc & 273 & 1,920 & 1 & IrMo & none & 3,140 & 1,820 & 44 & none & & 2,020 & 1 \\
\hline FeIrNi & fcc & 100 & 880 & 34 & fcc & 570 & 2,040 & 2 & FeIrRh & fcc & 1,420 & 740 & 43 & fcc & 690 & 180 & 1 \\
\hline FeIrRu & none & 2,850 & 1,240 & 33 & fcc & 1,800 & 2,380 & 2 & eMnMo & none & 2,050 & 1,620 & 32 & none & & 1,510 & 3 \\
\hline $\mathrm{nNb}$ & ne & 3,330 & 2,330 & 35 & none & & 1,530 & 2 & & one & 1,830 & 1,120 & 37 & fcc & 550 & 1,500 & 2 \\
\hline $\mathrm{aRh}$ & ne & 2,360 & 1,800 & 41 & fcc & 273 & 1,790 & 1 & $\mathrm{Ta}$ & one & 4,650 & 2,740 & 37 & none & & 1,650 & - \\
\hline & ne & 170 & 2,490 & 48 & one & 273 & 1,590 & 3 & & one & 2,820 & 1,400 & 25 & $\mathrm{bcc}$ & 1,440 & 1,670 & - \\
\hline & ne & 2,880 & 1,550 & 8 & one & & 1,650 & 3 & & fcc & 1,080 & 1,480 & 47 & one & & 1,630 & - \\
\hline & ne & 130 & 1,520 & 49 & one & & 1,530 & 3 & & none & 2,500 & 1,630 & 46 & one & & 1,890 & - \\
\hline & ne & 200 & 810 & 11 & none & & 1,730 & 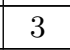 & & none & 4,780 & 2,540 & 47 & one & & 1,530 & 3 \\
\hline & none & 2,260 & 180 & 31 & $\mathrm{bcc}$ & U & 1,940 & 3 & & none & 2,930 & 1,540 & 50 & none & & 1,930 & 3 \\
\hline $\mathrm{Ta}$ & none & 2,200 & 1,210 & 32 & none & & 1,610 & 3 & & none & 4,840 & 2,620 & 40 & none & & 1,320 & 3 \\
\hline & none & 2,000 & 1,210 & 27 & none & & 1,540 & 3 & $\mathrm{Rh}$ & none & 2,000 & 1,250 & 38 & fcc & 550 & 1,900 & 1 \\
\hline FeTaTi & none & 4,700 & 2,640 & 43 & none & & 1,350 & 3 & & none & 2,170 & 1,730 & 31 & none & & 1,670 & 5 \\
\hline FeTaW & none & 3,200 & 1,730 & 47 & none & & 1,960 & 3 & & none & 4,650 & 2,180 & 33 & bcc & 310 & 1,480 & 3 \\
\hline & none & 2,590 & 1,260 & 39 & none & & 1,880 & 3 & & bcc & 1,760 & 980 & 20 & $\mathrm{bcc}$ &, 370 & 2,280 & 3 \\
\hline $\mathrm{oRe}$ & one & 4,440 & 2,600 & 48 & $\mathrm{bcc}$ & 0 & 2,350 & 1 & & bcc & 1,720 & 1,510 & 2 & Dcc & 640 & 2,280 & 3 \\
\hline & bcc & \begin{tabular}{|l|}
1,890 \\
\end{tabular} & 1,120 & 31 & $\mathrm{bcc}$ & & 2,100 & 3 & & $\mathrm{bcc}$ & 2,100 & 1,440 & $3 r$ & $\mathrm{cc}$ & 1,470 & 2,290 & 2 \\
\hline & one & 3,340 & 2,190 & 36 & none & & 2,540 & & & none & 5,670 & 3,400 & 4 & bcc & 1,860 & 1,990 & - \\
\hline & bcc & 640 & 860 & 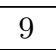 & $\mathrm{bcc}$ & 370 & 2,490 & 3 & & bcc & 1,320 & 880 & 2 & bcc & ,11 & 2,130 & 3 \\
\hline & bcc & 670 & 1,700 & 23 & bcc & 090 & 2,240 & 2 & & bcc & 940 & 1,100 & 18 & bcc & 2,040 & 2,250 & 3 \\
\hline & bcc & 730 & 180 & 2 & bcc & 1,250 & 2,190 & 3 & & none & 4,520 & 2,350 & 38 & 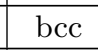 & 180 & 2,610 & 1 \\
\hline & $\mathrm{bcc}$ & 1,560 & 640 & 44 & $\mathrm{bcc}$ & 2,100 & 2,300 & 1 & & $\mathrm{bcc}$ & 1,840 & 1,230 & 19 & $\mathrm{cc}$ & 1,150 & 2,200 & 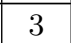 \\
\hline & bcc & 1,790 & 2,080 & 18 & $\mathrm{bcc}$ & 1,170 & 2,380 & 2 & & bcc & 1,280 & 1,410 & 17 & bcc & 1,960 & 2,320 & 3 \\
\hline & bcc & 2,200 & 1,480 & 2 & $\mathrm{bcc}$ & 630 & 2,250 & 3 & & bcc & 1,690 & 1,490 & 31 & bcc & 870 & 2,050 & 2 \\
\hline $\mathrm{iW}$ & bcc & 1,580 & 1,730 & 29 & none & & 2,110 & 3 & & bcc & 1,630 & 1,270 & 36 & bcc & 1,240 & 2,000 & 3 \\
\hline $\mathrm{VW}$ & bcc & 2,110 & 1,620 & 44 & $\mathrm{bcc}$ & 2,190 & 2,270 & 2 & & none & 2,570 & 1,930 & 33 & bcc & 1,400 & 1,920 & 2 \\
\hline & none & 4,900 & 2,870 & 4 & none & & 2,050 & 3 & & one & 2,410 & 1,720 & 2 & fcc & 740 & 1,690 & 1 \\
\hline & ne & 1,990 & 500 & & $\mathrm{fc}$ & & 1,990 & 1 & & none & 2,680 & 1,660 & 37 & non & & $|1,950|$ & 2 \\
\hline & ne & 3,070 & 2,020 & & fcc & 0 & 2,23 & 2 & & fcc & 1,660 & & 50 & or & & 2,030 & 1 \\
\hline & none & 2,410 & 3,130 & 49 & none & & 1,860 & 1 & & none & 1,830 & 1,140 & 21 & $\mathrm{fcc}$ & 1,330 & 1,670 & 3 \\
\hline & fcc & 470 & 830 & 2 & fcc & 050 & 2,010 & 2 & & fcc & 730 & 490 & 17 & ICC & 700 & 2,090 & 2 \\
\hline & fo & 470 & 970 & 1 & fcc & 520 & 2,430 & 3 & & none & 2,440 & 1,820 & 44 & none & & 1,980 & 2 \\
\hline & & 480 & 1,010 & 1 & fcc & 1,830 & 2,620 & 1 & & fcc & 1,990 & 940 & 12 & fcc & 450 & 2,020 & 2 \\
\hline IrPdRh & fcc & 1,980 & 880 & 12 & fcc & 1,280 & 2,090 & 3 & & none & 2,840 & 1,440 & 17 & fcc & 820 & 2,150 & 3 \\
\hline & none & 2,950 & 2,210 & 46 & fcc & 1,220 & 1,730 & 1 & & none & 3,280 & 2,470 & 41 & none & & 210 & 1 \\
\hline tRe & fcc & 1,610 & 1,510 & 26 & fcc & 1,790 & 2,410 & - & & fcc & 500 & 470 & 6 & fcc & ,310 & 2,190 & 3 \\
\hline IrPtRu & fcc & 1,120 & 690 & 12 & fcc & 990 & 2,630 & 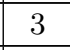 & $\mathrm{PtTc}$ & fcc & 1,520 & 1,340 & 20 & fcc & 1,540 & 2,110 & 1 \\
\hline IrRhV & none & 3,770 & 1,920 & 45 & fcc & 790 & 1,780 & 1 & $\mathrm{IoNb}$ & none & 3,210 & 1,630 & 25 & bcc & 1,430 & 2,220 & 2 \\
\hline $\mathrm{Ta}$ & 1 & 4,340 & 40 & 2 & $\mathrm{bcc}$ & 0 & 2 & 2 & $\mathrm{Ti}$ & none & 2,970 & 1,860 & 30 & one & & 1,550 & $\tau$ \\
\hline & & 20 & 10 & 2 & $\mathrm{~b}$ & 50 & & 3 & & DCc & 000 & 1,660 & 0 & ione & 970 & 2,010 & 3 \\
\hline & none & 5,070 & 2,040 & 2 & DCC & 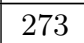 & 2,460 & 1 & & one & 3,580 & 2,320 & 41 & $\mathrm{bcc}$ & 80 & 1,830 & 2 \\
\hline & none & 3,330 & 1,950 & 2 & $\mathrm{~b}$ & 2 & r & 2 & & none & 3,41 & 1,590 & 2 & none & & 1,780 & - \\
\hline & one & 810 & 1,940 & 3 & bcc & 990 & 1,910 & 2 & & none & 4,400 & 2,070 & 1 & bcc & 70 & 2,110 & 2 \\
\hline & none & 4,510 & 2,110 & 31 & none & & 1,880 & 2 & & none & 2,930 & 2,440 & 42 & bcc & 850 & 1,640 & 3 \\
\hline TiW & none & 3,900 & 2,530 & 40 & none & 850 & 2,330 & 3 & VW & none & 3,030 & 1,680 & 22 & none & & 1,820 & 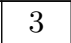 \\
\hline MoNbRe & bcc & 2,540 & 1,410 & 14 & $\mathrm{bcc}$ & 1,840 & 2,790 & - & $\mathrm{NbTa}$ & bcc & 1,080 & 660 & 4 & bcc & 273 &, 910 & 3 \\
\hline $\mathrm{bTc}$ & none & 3,590 & 1,820 & 16 & $\mathrm{bcc}$ & 1,790 & 2,410 & 1 & $\mathrm{IbTi}$ & bcc & 790 & 560 & 14 & bcc & 990 & 2,430 & 3 \\
\hline MoNbV & bcc & 940 & 680 & 5 & bcc & 1,310 & 2,430 & 3 & MoNbW & $\mathrm{bcc}$ & 690 & 540 & 3 & $\mathrm{bcc}$ & 273 & 3,030 & 3 \\
\hline
\end{tabular}




\begin{tabular}{|c|c|c|c|c|c|c|c|c|c|c|c|c|c|c|c|c|c|}
\hline & N. & $T_{\mathrm{c}}$ & $T_{\mathrm{c}}$ & & & c & $T_{\mathrm{m}}^{(\star)}$ & & & D.R. & $T_{\mathrm{c}}$ & $\tilde{T}_{\mathrm{c}}$ & & & $T_{\mathrm{c}}^{(\star)}$ & $T_{\mathrm{m}}^{(\star)}$ & $\cdots$ \\
\hline & one & 2,570 & 1,810 & 20 & $\mathrm{bcc}$ & 810 & 1,930 & 2 & & one & & 1,450 & 42 & none & & 400 & \\
\hline & 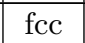 & & 280 & & one & & 760 & & & 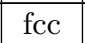 & & 850 & & one & & 830 & 1 \\
\hline & fcc & 660 & 420 & & $\overline{f c c}$ & 700 & \begin{tabular}{|l|l|}
1,920 \\
\end{tabular} & & & $\mathrm{c}$ & 780 & 600 & 32 & $\mathrm{cc}$ & 590 & 000 & 2 \\
\hline$\Gamma \mathrm{a}$ & one & 3,950 & 950 & & $\mathrm{bcc}$ & 100 & 2,950 & & & $\mathrm{bcc}$ & 1,310 & 1,740 & 28 & $\mathrm{cc}$ & 030 & 430 & 1 \\
\hline $\mathrm{MoF}$ & bcc & 740 & 1,310 & & $\mathrm{bcc}$ & 1,950 & 2,520 & & & bcc & 820 & 880 & & $\mathrm{cc}$ & 620 & ,150 & 1 \\
\hline $\mathrm{Tc}$ & none & 330 & 2,400 & 2 & $\mathrm{bcc}$ & 2,040 & 2,480 & & & bcc & 810 & 10 & 14 & $\mathrm{cc}$ & 310 & ,480 & 3 \\
\hline & $\mathrm{cc}$ & 200 & 070 & & $\mathrm{bcc}$ & 1,790 & 2,440 & & & bcc & 730 & 840 & & $\mathrm{ccc}$ & 3 & 200 & - \\
\hline & 4 & 660 & 760 & 4 & none & & 970 & & & one & 2,750 & 1,980 & & $\mathrm{bcc}$ & &, 130 & 1 \\
\hline & $c_{1}$ & 500 & 540 & & 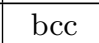 & 0 & 2,230 & & & $\mathrm{bcc}$ & & & & bcc & &, 710 & 1 \\
\hline & bcc & 50 & 650 & & bcc & 110 & 2,220 & & & $\mathrm{bcc}$ & 800 & 50 & & occ & & 2,580 & 3 \\
\hline & $\mathrm{bcc}$ & 160 & 200 & & b & 210 & 1,840 & & & $b c c$ & 410 & 30 & & DCC & & 2,720 & 3 \\
\hline & ne & 50 & 00 & & $\mathrm{bcc}$ & & 1,670 & & & one & 2,470 & 170 & & one & & , 830 & 3 \\
\hline & 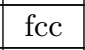 & 70 & 540 & & one & & 790 & & & one & 180 & 1,710 & & $\mathrm{CC}$ & & 2,920 & 1 \\
\hline & $\mathrm{cc}$ & 330 & 420 & & bcc & 130 & 2,290 & & & $\mathrm{bcc}$ & 1,890 & 1,560 & & cc & 700 & 300 & 1 \\
\hline & $\mathrm{cc}$ & 620 & 530 & & $\mathrm{cc}$ & 740 & 3,050 & & & none & 2,940 & 1,920 & & $\mathrm{cc}$ & 180 &, 220 & 1 \\
\hline & ne & 5,400 & 1,990 & & $\mathrm{bcc}$ & 1,530 & 2,500 & & & $\mathrm{bcc}$ & 640 & 460 & & $\mathrm{ccc}$ & 20 &, 430 & 3 \\
\hline & $b c c$ & 410 & 990 & 9 & bcc & 900 & 2,400 & & & $\mathrm{bcc}$ & 300 & 20 & & cc & 73 &, 160 & 3 \\
\hline & $\mathrm{bcc}$ & 470 & 940 & 8 & $\mathrm{bcc}$ & 1,590 & 2,170 & & & none & 3,400 & 1,770 & 2 & $\mathrm{cc}$ & 1,660 & 2,060 & 1 \\
\hline & nne & 3,600 & 1,940 & 2 & $\mathrm{bcc}$ & 700 & 2,620 & & & $\mathrm{bcc}$ & 110 & 90 & & $\mathrm{cc}$ & 650 & ,030 & 3 \\
\hline & 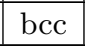 & 30 & 450 & 1 & b & 420 & 100 & & & bcc & 50 & 0 & & $\mathrm{cc}$ & &, 960 & 3 \\
\hline & bcc & 880 & 620 & 6 & b & & 2,500 & & & ione & 0 & & & $\mathrm{CC}$ & 0 & 00 & 3 \\
\hline & ne & 90 & $|1,380|$ & & II & & 1,980 & & & fcc & 1,1 & & & & & ,670 & 1 \\
\hline & & 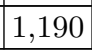 & \begin{tabular}{|l|}
720 \\
\end{tabular} & & & & $1,6^{\prime}$ & & & none & 1,6 & 1,600 & & c & & 1,450 & 2 \\
\hline & & 050 & 630 & & & 273 & 2,02 & & & fcc & 1,2 & 8 & & & & 2,060 & 2 \\
\hline & & 180 & 900 & & & 090 & 1,630 & & & none & 3,260 & 40 & & & & 1,680 & 1 \\
\hline & & 750 & 910 & & ne & & 1,880 & & & fcc & 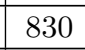 & & & & & 2,040 & 2 \\
\hline & & 170 & 70 & 2 & $c_{1}$ & 250 & 2,060 & & & fcc & 030 & 2,900 & 39 & one & & 1,780 & 1 \\
\hline & fcc & 520 & 1,740 & 4 & & 130 & 1,650 & & & fcc & 740 & 800 & 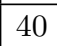 & one & & 050 & 1 \\
\hline & fcc & 220 & 1,760 & 39 & $\mathrm{cc}$ & 1,740 & 2,480 & & & none & 2,340 & 1,620 & $D_{0}$ & $\mathrm{bcc}$ & 30 & 1,520 & 1 \\
\hline & fcc & 770 & 530 & 9 & fcc & 940 & 2,290 & & & none & 2,080 & 1,860 & . & none & & 1,760 & 1 \\
\hline & fcc & 410 & 2,000 & 4 & none & & 190 & & & none & 2,980 & 1,680 & & $\mathrm{bcc}$ & & 2,430 & 1 \\
\hline & ne & 3,520 & 1,990 & & & 740 & 2,500 & & & none & 3,920 & 2,110 & & cc & & 3,340 & 1 \\
\hline & ne & 4,120 & 2,440 & & & 220 & 2,320 & & & $\mathrm{bcc}$ & 2,320 & 1,870 & & bcc & & 2,540 & 1 \\
\hline & & 60 & t & & & 70 & 2, & & & one & 4,6 & & & & & 2,120 & 1 \\
\hline & ne & 420 & 30 & 2 & $\mathrm{~b}$ & 1,700 & 2,190 & & & none & 5,010 & 2,580 & & bcc & & 2,820 & 1 \\
\hline & $b_{1}$ & 380 & 450 & 7 & $\mathrm{~b}$ & & & & & $\mathrm{bc}$ & & & 1 & bcc & & 2,540 & 3 \\
\hline & $D C$ & 330 & 110 & & $\mathrm{CC}$ & 410 & 1,980 & & & $\mathrm{bc}$ & 75 & & & $\mathrm{bc}$ & & 2,680 & 3 \\
\hline & one & 1,940 & 2,210 & 1 & none & & 670 & & & non & 2,88 & 1,690 & -1 & non & &, 050 & 3 \\
\hline & one & 3,140 & 2,250 & 3 & $\mathrm{bcc}$ & $J 0$ & 2,230 & & & none & 2,440 & 1,590 & & $\mathrm{bcc}$ & & 2,340 & 1 \\
\hline & none & 2,200 & 1,580 & 40 & $\mathrm{bcc}$ & 1,330 & 1,550 & 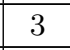 & & none & 960 & 1,730 & 24 & none & & 880 & 3 \\
\hline VWZr & \begin{tabular}{|l} 
none \\
\end{tabular} & 3,710 & \begin{tabular}{|l|l|}
2,040 \\
\end{tabular} & 28 & $\begin{array}{l}\text { none } \\
\end{array}$ & & 1,640 & 3 & & & & & & & & & \\
\hline
\end{tabular}




\section{Quaternary alloys at equi-composition}

TABLE V: LTVC results for quaternary alloys. S.S. type of single solid solution phase ("none" = not found); $T_{\mathrm{c}}(\mathrm{K})$ transition temperature, as estimated by the method; $\tilde{T}_{\mathrm{c}}(\mathrm{K})$ approximated transition temperature, estimated according to Eq. (8); CV $(\mathrm{meV})$ cluster expansion cross validation score; $\bar{T}_{\mathrm{m}}(\mathrm{K})$ melting temperature (solidus), calculated as the average of the melting temperatures at equi-composition of the 6 binary alloys associated with the quaternary alloy; A solid solution is predicted if $T_{\mathrm{c}}<\bar{T}_{\mathrm{m}}$.

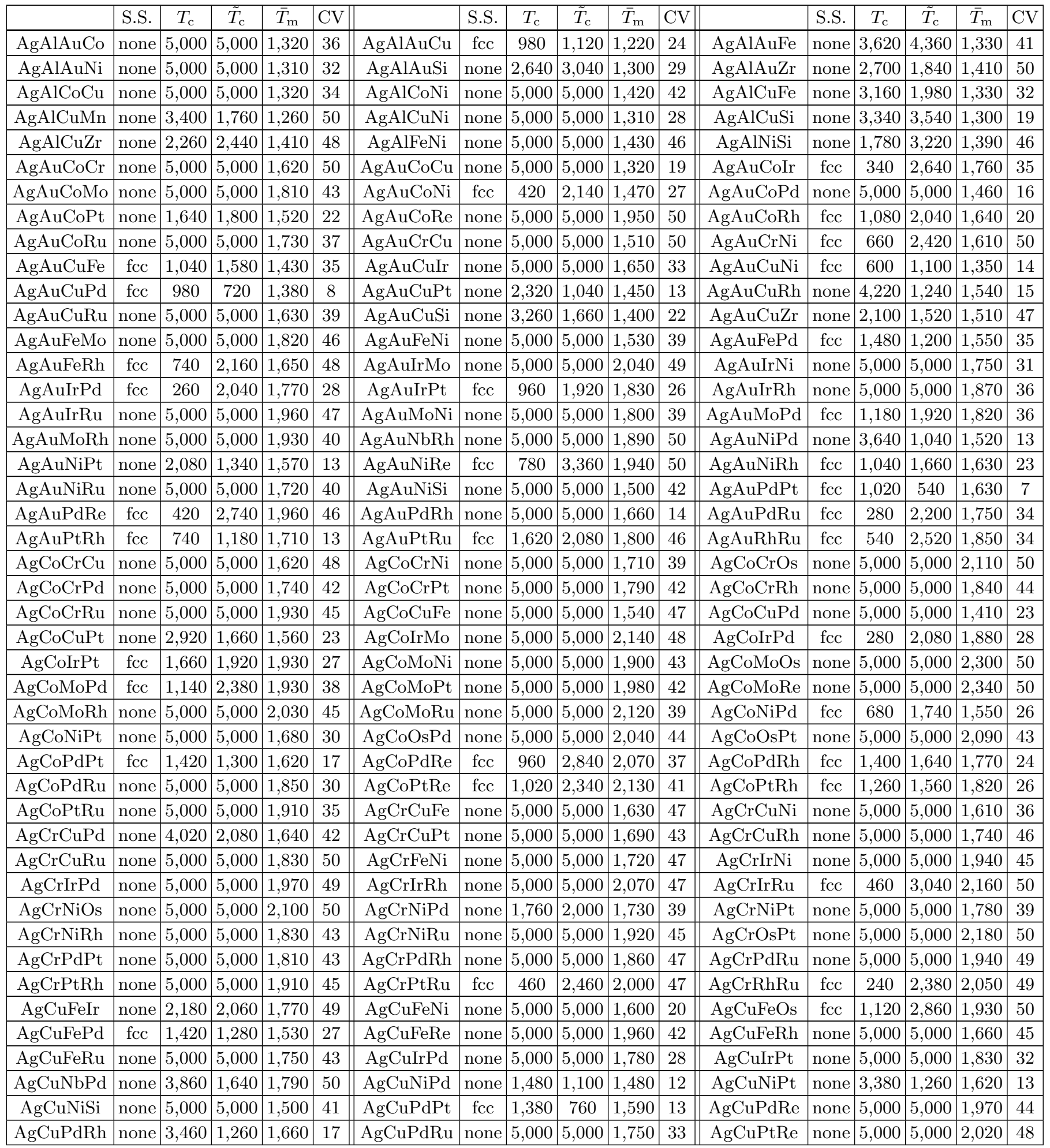




\begin{tabular}{|c|c|c|c|c|c|c|c|c|c|c|c|c|c|c|c|c|c|}
\hline & S. & $T_{\mathrm{c}}$ & $\tilde{T}_{\mathrm{c}}$ & $\bar{T}_{\mathrm{m}}$ & $\mathrm{CV}$ & & S.S. & $T_{\mathrm{c}}$ & $\tilde{T}_{\mathrm{c}}$ & $\Lambda_{\mathrm{m}}$ & 年 & & S.S. & $T_{\mathrm{c}}$ & $\tilde{T}_{\mathrm{c}}$ & $\bar{T}_{\mathrm{m}}$ & $\mathrm{CV}$ \\
\hline $\mathrm{Rh}$ & ne & 2,900 & 1,260 & 1,720 & 21 & $\mathrm{tRu}$ & none & 5,000 & 5,000 & 1,800 & 42 & & one & 5,000 & 5,000 & 860 & 45 \\
\hline & one & 5,000 & 000 & 80 & 50 & & none & 000 & 5,000 & 1,910 & 42 & & $\mathrm{cc}$ & ,320 & 240 & 310 & 48 \\
\hline $\mathrm{oPd}$ & fcc & 720 & 180 & 40 & 43 & & fcc & 440 & 3,220 & 2,350 & 411 & & ne & 5,000 & & 040 & 50 \\
\hline $\mathrm{oRu}$ & none & 5,000 & 000 & 2,130 & \begin{tabular}{|l|}
47 \\
\end{tabular} & & none & 5,000 & 5,000 & 2,020 & 44 & & 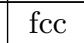 & 700 & 1,740 & 1,650 & 41 \\
\hline & none & 5,000 & 5,000 & 2,060 & 42 & & none & 5,000 & 5,000 & 1,750 & 41 & & none & 5,000 & 5,000 & ,840 & 40 \\
\hline $\mathrm{sPd}$ & none & 5,000 & 5,000 & 2,050 & 45 & sRe & fcc & 1,360 & 3,480 & 2,450 & 47 & OsRh & none & 5,000 & 5,000 & 2,150 & 50 \\
\hline $\mathrm{sRu}$ & fcc & 1,360 & 3,160 & 2,240 & 48 & $\mathrm{dRe}$ & none & 5,000 & 5,000 & 2,080 & 44 & $\mathrm{dRh}$ & fec & 720 & 1,720 & 1,780 & 47 \\
\hline $\mathrm{dRu}$ & one & 5,000 & 5,000 & 1,860 & 41 & eRu & fcc & 1,280 & 3,200 & 2,270 & 46 & $\mathrm{hRRu}$ & none & 5,000 & 5,000 & 1,970 & 50 \\
\hline & one & 5,000 & 5,000 & 2,130 & 42 & & none & 5,000 & 5,000 & 2,160 & 11 & & none & 5,000 & 5,000 & 2,260 & 47 \\
\hline & one & 5,000 & 000 & 180 & 50 & & fcc & 1,240 & 1,900 & 1,870 & 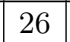 & & fcc & 1,620 & 1,700 & 1,92 & 24 \\
\hline & fcc & 440 & 860 & 270 & 47 & & none & 5,000 & 5,000 & 2,320 & & & $\mathrm{n}$ & 5,000 & 5,000 &, 95 & 21 \\
\hline & ne & 5,000 & 000 & 300 & 11 & & ne & 5,000 & 5,000 & 2,000 & & & & 420 & 00 & 00 & 32 \\
\hline & fcc & 1,040 & 040 & 2,350 & 42 & & fcc & 380 & 1,500 & 2,050 & & & 100 & 1,240 & 2,080 & 2,140 & 41 \\
\hline & ne & 5,000 & 000 & 2,290 & 50 & & nne & 5,000 & 5,000 & 1,920 & & & none & 5,000 & 5,000 & $1,9 \pi$ & 42 \\
\hline & nne & 5,000 & 5,000 & 2,330 & 45 & & none & 5,000 & 5,000 & 2,020 & 37 & & fcc & 780 & 840 &, 110 & 41 \\
\hline & none & 5,000 & 5,000 & 2,320 & 47 & & fcc & 1,600 & 3,580 & 2,720 & 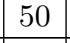 & & none & 5,000 & 5,000 & 2,420 & 50 \\
\hline $\mathrm{dPt}$ & one & 5,000 & 5,000 & 2,000 & 40 & & fcc & 1,740 & 2,700 & 2,350 & 42 & & none & 5,000 & 5,000 & 2,050 & 35 \\
\hline $\mathrm{Ru}$ & none & 5,000 & 5,000 & 2,130 & 40 & & none & 5,000 & 5,000 & 2,410 & D & & none & 5,000 & 5,000 & 2,10 & 50 \\
\hline $\mathrm{tRu}$ & none & 5,000 & 5,000 & 2,190 & 50 & & none & 5,000 & 5,000 & 2,450 & 42 & & nol & 5,000 & 5,000 & 2,24 & 45 \\
\hline & ne & 5,000 & 5,000 & 1,960 & 50 & & ne & 5,000 & 5,000 & 2,010 & 49 & & nol & 5,000 & 5,000 & 2,070 & 48 \\
\hline & fcc & 460 & 2,640 & 2,030 & 50 & & none & 5,000 & 5,000 & 2,080 & & & fcc & 1,580 & 1,080 & 1,700 & 12 \\
\hline & fcc & 1,960 & 2,580 & 2,060 & 40 & & fcc & 720 & 1,360 & 1,760 & & & none & 5,000 & 00 & , 84 & 28 \\
\hline & fcc & 920 & 2,220 & 110 & 44 & & fcc & 1,200 & 1,32 & 1,810 & 1 & & fcc & 560 & 840 & (96 & 33 \\
\hline & one & 5,000 & 000 & 10 & 6 & & cc & 960 & 3,560 & 2,460 & & & $\mathrm{Cc}$ & 480 & 2,480 &, 15 & 50 \\
\hline & fcc & 740 & 3,180 & 240 & 42 & & $c c$ & 760 & 3,080 & 2,510 & $x_{0}$ & & ICC & 1,380 & 2,12 & 2,21 & 50 \\
\hline & fcc & 760 & 2,720 & 300 & - & & fcc & 1,720 & 2,120 & 2,140 & 40 & & nol & 5,000 & 5,000 & 1,84 & 10 \\
\hline & fo & 1,140 & 640 & 920 & 26 & & fcc & 720 & 2,740 & 2,190 & 00 & & none & 5,000 & 5,000 & 2,270 & 40 \\
\hline & fcc & 540 & 2,020 & 1,970 & 29 & & fcc & 1,140 & 2,300 & 2,240 & & & fcc & 540 & 2,500 & 2,330 & 42 \\
\hline & fcc & 820 & 1,720 & 2,030 & 28 & & ne & 3,960 & 2,380 & 1,630 & 49 & & nol & 3,680 & 1,860 & 1,600 & 49 \\
\hline & none & 2,000 & 1,200 & 1,620 & 42 & & none & 2,180 & 1,340 & 1,870 & r & & none & 3,820 & 1,880 & 1,700 & 50 \\
\hline & none & 2,080 & 1,400 & 2,080 & 49 & & none & 3,160 & 1,720 & 1,660 & 8 & & none & 3,120 & 2,040 & 1,790 & 45 \\
\hline & none & 2,980 & 1,520 & 1,890 & 50 & & $\mathrm{bcc}$ & 1,740 & 1,300 & 1,860 & $\pi$ & & 101 & 2,320 & 1,900 & 2,160 & 50 \\
\hline Mo & none & 1,920 & 060 & 1,790 & 47 & & none & 2,080 & 1,500 & 1,860 & 4 & & hon & 2,300 & 1,540 & $\mid 1,610$ & 48 \\
\hline & none & 2,540 & 220 & 770 & 4 & & none & 4,260 & 1,960 & 2,070 & 4 & & $b^{2}$ & 1,260 & 1,240 & 2,22 & 45 \\
\hline & bcc & 860 & 240 & 920 & & & $\mathrm{bcc}$ & 1,260 & 880 & 050 & & & $\mathrm{cc}$ & 1,500 & 960 & 2,2 & 45 \\
\hline & ne & 180 & 60 & 40 & & & none & 2,660 & 1,800 & 2,020 & & & $\mathrm{Cc}$ & 1,600 & 1,340 & 2,1 & 48 \\
\hline & $\mathrm{b}$ & 140 & 340 & 40 & & & $\mathrm{n}$ & 2,080 & 1,380 & 1,830 & & & $\mathrm{Cc}$ & 1,860 & 1,260 & 1,9 & 48 \\
\hline & 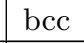 & 440 & 380 & 120 & 1. & & none & 2,460 & 1,420 & 1,430 & $\pi$ & & bcc & 1,380 & 1,440 & $1,5 \varepsilon$ & 50 \\
\hline & none & 3,900 & 1,860 & 1,720 & 50 & & none & 2,320 & 1,460 & 1,930 & 50 & & Inone & 4,040 & 2,980 & 1,570 & 50 \\
\hline & none & 3,620 & 1,800 & 1,690 & 47 & & none & 2,700 & 1,260 & 1,740 & 50 & & $\mathrm{bcc}$ & 1,660 & 1,160 & 1,900 & 50 \\
\hline & bcc & 1,380 & 1,700 & 2,120 & 50 & & none & 2,200 & 1,820 & 2,170 & 50 & $o \operatorname{Re}$ & none & 2,300 & 1,540 & 1,970 & 50 \\
\hline eW & none & 2,640 & 1,700 & 2,400 & 49 & & $\mathrm{bcc}$ & 1,280 & 1,260 & 1,730 & 50 & & none & 2,680 & 1,360 & 2,070 & 48 \\
\hline & $\mathrm{bcc}$ & 1,280 & 1,060 & 2,040 & 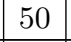 & & bcc & 1,100 & 760 & 2,340 & 49 & & bcc & 1,260 & 1,060 & 1,970 & 50 \\
\hline & $\mathrm{bcc}$ & 580 & 740 & 2,240 & 5 & & $\mathrm{bcc}$ & 1,720 & 1,360 & 2,410 & 50 & & $\mathrm{bcc}$ & 1,760 & 1,080 & 2,050 & 46 \\
\hline $\mathrm{Pd}$ & $\mathrm{fe}$ & 720 & 820 & 770 & 48 & & none & 5,000 & 5,000 & 1,820 & 4 & & nol & 4,700 & 1,380 & 1,500 & 18 \\
\hline & ne & 1,840 & 300 & 560 & 1 & & fo & 640 & 1,760 & 1,900 & 2 & & 10 & 2,360 & 1,740 & 1,960 & 22 \\
\hline & 100 & 200 & 860 & 960 & 3 & & none & 5,000 & 5,00 & 2,010 & & & ICC & 520 & 1,380 & 1,53 & 26 \\
\hline & one & 4,200 & 380 & 580 & 2 & & none & 5,000 & 5,00 & 2,060 & 50 & & nol & 5,000 & 5,00 & 2,12 & 35 \\
\hline & one & 1,940 & 220 & 640 & 13 & & nor & 5,000 & 5,00 & 2,100 & 38 & & no1 & 3,900 & 1,340 & $1, r s$ & 20 \\
\hline $\mathrm{Ru}$ & one & 5,000 & 5,000 & 1,880 & 35 & & none & 5,000 & 5,000 & 2,150 & 35 & $\mathrm{Rh}$ & not & 4,220 & 1,260 & 1,850 & 16 \\
\hline & none & 5,000 & 5,000 & 1,930 & 28 & & none & 4,080 & 1,540 & 1,660 & 49 & & hone & 5,000 & 5,000 & 1,720 & 47 \\
\hline & none & 5,000 & 5,000 & 1,990 & 48 & & none & 5,000 & 5,000 & 2,050 & 42 & $\mathrm{iPd}$ & none & 3,280 & 1,560 & 1,760 & 46 \\
\hline $\mathrm{AuCrNiPt}$ & none & 5,000 & 5,000 & 1,810 & 42 & $\mathrm{AuCrOsPt}$ & none & 5,000 & 5,000 & 2,210 & 50 & $\mathrm{AuCrPdPt}$ & fcc & 560 & 1,740 & 1,830 & 47 \\
\hline
\end{tabular}




\begin{tabular}{|c|c|c|c|c|c|c|c|c|c|c|c|c|c|c|c|c|c|}
\hline & S. & $T_{\mathrm{c}}$ & $\tilde{T}_{\mathrm{c}}$ & $\bar{T}_{\mathrm{m}}$ & $\mathrm{CV}$ & & S.S. & $T_{\mathrm{c}}$ & $\tilde{T}_{\mathrm{c}}$ & $\bar{T}_{\mathrm{m}}$ & $\mathrm{CV}$ & & . & $I_{\mathrm{C}}$ & $\tilde{T}_{\mathrm{c}}$ & $\bar{T}_{\mathrm{m}}$ & $\mathrm{CV}$ \\
\hline & ne & 5,000 & 5,000 & 1,880 & 46 & & none & 5,000 & 5,000 & 1,970 & 50 & (1111 & one & 000 & 5,000 & 1,940 & 40 \\
\hline & one & 000 & 000 & 2,020 & 46 & & fcc & 320 & 00 &, 580 & 40 & & one & & 000 & 800 & 27 \\
\hline & one & 5,000 & 500 & 1,860 & 24 & & none & 2,140 & 880 & 1,450 & 184 & & one & 300 & 1,020 &, 500 & 14 \\
\hline & one & 5,000 & 5,000 & 2,010 & 47 & & none & 1,620 & 780 & 1,580 & 10 & $\mathrm{dRe}$ & fcc & 460 & 2,180 & ,990 & 50 \\
\hline $\mathrm{dRh}$ & none & 3,820 & 1,120 & 1,690 & 15 & $\mathrm{AuC}$ & fcc & 500 & 1,880 & 1,780 & 43 & tRe & fcc & 760 & 2,440 & 2,050 & 46 \\
\hline $\mathrm{AuCuPtRh}$ & none & 1,960 & 1,040 & 1,740 & 16 & AuCuPtRu & fcc & 1,520 & 1,680 & 1,830 & 33 & $\mathrm{IoPd}$ & none & 2,280 & 1,580 & 1,970 & 49 \\
\hline $\mathrm{AuFe}$ & fcc & 940 & 1,160 & 1,670 & 48 & AuFeOsPd & none & 5,000 & 5,000 & 2,070 & 50 & dRe & fcc & 540 & 2,100 & 2,110 & 48 \\
\hline AuFePdRh & one & 5,000 & 5,000 & 1,800 & 50 & $\mathrm{dRu}$ & none & 5,000 & 5,000 & 1,890 & 46 & $\mathrm{oPd}$ & none & 5,000 & 5,000 & 2,180 & 37 \\
\hline & fcc & 980 & 1,760 & 890 & 24 & & none & 2,060 & 1,560 & 1,950 & 23 & & fcc & 500 & 2,760 & 2,290 & 45 \\
\hline & fcc & 940 & 220 & 350 & 40 & & fcc & 340 & 500 & 1,970 & & & one & 5,000 & 000 & 2,33 & 38 \\
\hline & fcc & 540 & 760 & 020 & 19 & & fcc & 800 & 360 & 2,110 & & & one & 5,000 & 000 & 38 & 39 \\
\hline & fcc & 380 & 320 & 080 & 10 & & fcc & 1,420 & 880 & 2,160 & 26 & & fcc & 660 & 1,000 & , & 37 \\
\hline & one & 000 & 000 & 000 & 42 & & none & 5,000 & 000 & 2,340 & 44 & & none & 5,000 & 00 & 021 & 39 \\
\hline & $\overline{f c c}$ & 440 & 160 & 380 & 8 & & none & 5,000 & 000 & 2,070 & 32 & & fcc &, 260 & 2,020 & 2,160 & 36 \\
\hline & one & 5,000 & 000 & 2,430 & 44 & & fcc & 340 & 920 & 2,130 & 50 & & none & 5,000 & 5,000 & 210 & 39 \\
\hline & none & 2,100 & 1,920 & 2,040 & 50 & & none & 5,000 & 5,000 & 2,090 & 50 & & fcc & 560 & 2,340 & 2,050 & 49 \\
\hline & none & 5,000 & 5,000 & 2,110 & 38 & & none & 2,700 & 1,080 & 1,600 & 12 & & none & 2,140 & 2,260 & 2,090 & 34 \\
\hline & none & 3,400 & 1,160 & 1,780 & 13 & & fcc & 620 & 1,800 & 1,870 & 32 & & fcc & 2,100 & 2,060 & 2,140 & 37 \\
\hline $\mathrm{Rh}$ & one & 3,320 & 1,100 & 1,840 & 14 & & none & 3,220 & 1,520 & 1,920 & 26 & & fcc & 1,060 & 2,120 & 2,130 & 36 \\
\hline & fcc & 1,020 & 300 & 2,480 & 46 & & fcc & 780 & 300 & 2,180 & . & & fcc & 1,080 & 1,900 & 2,230 & 45 \\
\hline & none & 5,000 & 000 & 320 & 37 & & fcc & 460 & 60 & 2,170 & 43 & & none & 3,060 & 940 & 1,860 & $J^{2}$ \\
\hline & fcc & 1,680 & 40 & 950 & 22 & & fcc & 900 &, 500 & is & 32 & & fcc & 980 & 720 & 30 & 37 \\
\hline & fcc & 940 & 800 & 2,000 & & & one & 5,000 & 5,000 & 2,270 & & & fcc & 0 & 2,320 & ,35 & 35 \\
\hline & fcc & 600 & 440 & 050 & & & none & 2,340 & 1,400 & 1,670 & 48 & & ione & 4,280 & 1,680 &, 62 & 34 \\
\hline & one & 3,360 & 420 & 780 & 1 & & fcc & 1,600 & 1,000 & 720 & 41 & & none & 4,2 & 1,680 & 1,79 & 33 \\
\hline & one & 2,020 & 20 & 950 & 1 & & none & 1,860 & 260 & 1,780 & 50 & & none & 1,920 & 1,300 & 1,68 & 50 \\
\hline & fcc & 280 & 020 & 580 & 77 & & $\mathrm{bcc}$ & 1,740 & 320 & 2,040 & 40 & & $\mathrm{bcc}$ & 1,640 & 1,340 & 2,39 & 44 \\
\hline & $\mathrm{cc}$ & 760 & 380 & 2,250 & 43 & & none & 3,080 & 1,140 & 1,600 & & & ione & 3,160 & 1,220 & 1,750 & 32 \\
\hline & one & 2,660 & 780 & 2,260 & 34 & & none & 2,560 & 1,500 & 2,320 & 33 & & none & 3,200 & 1,320 & 1,720 & 35 \\
\hline & one & 3,080 & 340 & 1,700 & 37 & & none & 2,900 & 1,340 & 2,080 & 34 & & none & 1,900 & 1,180 & 1,850 & 32 \\
\hline & fcc & 1,860 & 1,300 & 2,130 & 32 & & none & 4,380 & 1,400 & 1,740 & 20 & & none & 3,360 & 1,240 & 1,880 & 25 \\
\hline & none & 2,900 & 980 & 1,570 & 22 & & fcc & 840 & 840 & 1,700 & 18 & & none & 5,000 & 5,000 & 2,070 & 40 \\
\hline & one & 3,320 & 920 & 2,120 & 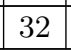 & & fcc & 1,640 & 960 & 1,670 & & & fcc & 1,440 & 2,040 & 2,1 & 34 \\
\hline & none & 2,820 & 1,220 & 1,630 & 28 & & none & 3,780 & 1,840 & 1,880 & 30 & & $\mathrm{cc}$ & 960 & 1,660 & 2,160 & 35 \\
\hline & fcc & 560 & 60 & 770 & 20 & & none & 3,320 & 460 & 1,940 & . & & $\mathrm{cc}$ & 1,550 & 900 & 1,60 & 48 \\
\hline & ne & 640 & 880 & 30 & & & none & 2,460 & 0 & 2, & & & one & 2,340 & 820 & 1,7 & 23 \\
\hline & one & 100 & 00 & 910 & & & $f_{f c c}$ & 580 & 0 & 2,400 & & & fcc & 2,380 & 1,180 & 2,4 & 16 \\
\hline & ne & 1,980 & 940 & 850 & 18 & & fcc & 2,000 & 640 & 2,430 & $1-2$ & & none & 2,26 & 800 & 1,9 & 19 \\
\hline & ne & 2,300 & 220 & 2,220 & $1:$ & & fcc & 2,440 & 620 & 2,490 & 2 & & fcc & 1,820 & 720 & 2,040 & 17 \\
\hline & none & 2,460 & 940 & 2,270 & 15 & & bcc & 2,000 & 1,340 & 2,550 & 47 & & none & 2,100 & 1,220 & 1,760 & 39 \\
\hline & none & 2,180 & 100 & 1,910 & 40 & & none & 2,820 & 1,740 & 2,450 & 31 & & fcc & 1,180 & 1,760 & 2,510 & 33 \\
\hline $\mathrm{dPt}$ & none & 1,960 & 280 & 1,900 & 38 & & fcc & 2,300 & 1,660 & 2,480 & 31 & $\mathrm{Rh}$ & none & 2,680 & 1,360 & 1,880 & 34 \\
\hline $\mathrm{Ru}$ & none & 2,500 & 1,560 & 2,270 & 31 & & fcc & 1,740 & 1,840 & 2,540 & 35 & $\mathrm{Rh}$ & fcc & 1,980 & 1,180 & 2,030 & 34 \\
\hline $\mathrm{tRu}$ & fcc & 2,240 & 1,400 & 2,320 & 32 & $\mathrm{Pd}$ & none & 2,820 & 1,300 & 2,160 & 26 & $\mathrm{Pt}$ & none & 2,280 & 1,140 & 2,210 & 30 \\
\hline & one & 1,800 & 700 & 680 & 24 & & fcc & 1,980 & 1,180 & 2,190 & 20 & & none & 2,320 & 800 & 1,690 & 25 \\
\hline & one & 3,540 & 1,140 & 1,980 & 2 & & fcc & 1,620 & 900 & 2,250 & 3 & & fcc & 1,720 & 660 & 1,82 & 20 \\
\hline & ne & 2,120 & 360 & 30 & 20 & & none & 2,660 & 1,480 & 2,240 & 25 & & $\mathrm{cc}$ & 1,220 & 1,760 & 2,59 & 24 \\
\hline & $\mathrm{fcc}$ & 920 & 280 & 90 & 24 & & fcc & 1,880 & 660 & 2, & & & $\mathrm{CC}$ & 900 & 1,460 & 2,6 & 23 \\
\hline & fcc & 780 & 100 & 2,340 & 21 & & fcc & 2,340 & 1,420 & 2,430 & 18 & & fec & 1,000 & 1,140 & 2,27 & 27 \\
\hline & fcc & 600 & 740 & 810 & 18 & & fcc & 1,040 & 1,020 & 2,060 & 20 & $\mathrm{Rh}$ & none & 2,320 & 1,420 & 2,32 & 27 \\
\hline $\mathrm{Ru}$ & fcc & 400 & 1,580 & 2,410 & 24 & $\mathrm{Ru}$ & none & 2,220 & 1,100 & 2,100 & 24 & & fcc & 980 & 1,000 & 2,380 & 26 \\
\hline $\mathrm{eRu}$ & fcc & 1,420 & 900 & 2,460 & 22 & & fcc & 1,400 & 840 & 2,160 & 15 & $\overline{\mathrm{Mn}}$ & none & 5,000 & 5,000 & 1,640 & 50 \\
\hline $\mathrm{CrCuFeMo}$ & none & 4,500 & 1,900 & 1,840 & 48 & $\mathrm{CrCuFeNb}$ & none & 3,660 & 1,840 & 1,850 & 47 & $\mathrm{CrCuFeNi}$ & none & 3,360 & 1,560 & 1,700 & 50 \\
\hline
\end{tabular}




\begin{tabular}{|c|c|c|c|c|c|c|c|c|c|c|c|c|c|c|c|c|c|}
\hline & S. & $T_{\mathrm{c}}$ & $\tilde{T}_{\mathrm{c}}$ & $\bar{T}_{\mathrm{m}}$ & $\mathrm{CV}$ & & & $T_{\mathrm{c}}$ & $\tilde{T}_{\mathrm{c}}$ & $\bar{T}_{\mathrm{m}}$ & 10 & & .N. & $I_{\mathrm{C}}$ & $\tilde{T}_{\mathrm{c}}$ & $\bar{T}_{\mathrm{m}}$ & CV \\
\hline & ne & 5,000 & 000 & 1,660 & 41 & & none & 4,920 & 1,800 & 910 & 36 & & one & 820 & 2,140 & 060 & 48 \\
\hline & one & 000 & 000 & 860 & 38 & & one & 000 & 5,000 & 020 & 37 & & one & & 000 & 800 & 47 \\
\hline & one & 5,000 & 00 & 2,170 & 50 & & one & 5,000 & 5,000 & 2,010 & 45 & & one & 380 & 1,920 & 1,780 & 50 \\
\hline $\mathrm{Cr}$ & one & 5,000 & 5,000 & 2,040 & 47 & & none & 5,000 & 5,000 & 2,290 & 50 & & none & 3,560 & 1,980 & 2,050 & 48 \\
\hline $\mathrm{CrCu}$ & none & 2,740 & 1,860 & 1,800 & 50 & $\mathrm{bV}$ & none & 3,520 & 1,700 & 2,050 & 44 & bW & none & 5,000 & 5,000 & 2,290 & 46 \\
\hline CrCuNiPd & none & 3,320 & 1,380 & 1,590 & 31 & $\mathrm{NiPt}$ & none & 3,240 & 1,360 & 1,740 & 30 & $\mathrm{OsPd}$ & fcc & 760 & 2,240 & 2,160 & 46 \\
\hline $\mathrm{CrCuOsPt}$ & fcc & 480 & 2,080 & 2,210 & 45 & $\mathrm{CrCuPdPt}$ & none & 4,000 & 1,460 & 1,740 & 38 & $\mathrm{PdRh}$ & none & 4,640 & 1,600 & 1,700 & 36 \\
\hline CrCuPdRu & one & 5,000 & 5,000 & 1,970 & 40 & $\mathrm{CrC}$ & one & 2,680 & 1,580 & 1,860 & 35 & PtRu & none & 3,680 & 1,680 & 2,030 & 40 \\
\hline $\mathrm{CrC}$ & ne & 2,840 & 2,100 & 810 & 48 & & none & 3,820 & 2,080 & 2,030 & 41 & & TH & 5,000 & 5,000 & 2,330 & 44 \\
\hline & none & 3,460 & 820 & 830 & 42 & & ione & 4,140 & 2,100 & 2,010 & & & one & 5,000 & 5,000 & 2,30 & 42 \\
\hline & one & 2,160 & 880 & 870 & 26 & & one &, 760 & 300 & 1,760 & & & one & 1,620 & 1,160 & 62 & 50 \\
\hline & $\mathrm{ccc}$ & 120 & 220 & 230 & 31 & & ne & 3,680 & 1,580 & 1,850 & & & & 5,000 & & 1,04 & 39 \\
\hline & $\mathrm{ccc}$ & 720 & 000 & 800 & 21 & & bcc & 2,100 & 1,180 & 2,280 & 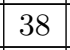 & & one & 2,360 & 000 &, 144 & 27 \\
\hline & bcc & 1,560 & 140 & 910 & 50 & & $\mathrm{cc}$ & 1,740 & 040 & 2,570 & 20 & & ione & 3,140 & 1,840 & 2,250 & 30 \\
\hline & one & 3,540 & 780 & 1,950 & 30 & & $\mathrm{cc}$ & 1,820 & 940 & 2,080 & t & & bcc & 2,220 & 1,220 & 310 & 34 \\
\hline & none & 2,600 & 1,520 & 2,530 & 34 & & none & 3,280 & 1,600 & 2,150 & 20 & & none & 4,420 & 2,140 & 1,840 & 34 \\
\hline & none & 2,520 & 1,320 & 1,950 & 22 & & $\mathrm{bcc}$ & 2,160 & 1,320 & 2,170 & & & none & 2,620 & 1,320 & 1,640 & 50 \\
\hline & bcc & 1,560 & 1,020 & 2,280 & 50 & & $\mathrm{bcc}$ & 1,420 & 800 & 1,740 & 49 & & fcc & 1,900 & 1,860 & 2,270 & 44 \\
\hline $\mathrm{CrF}$ & fcc & 1,880 & 1,760 & 2,090 & 43 & & none & 3,660 & 1,860 & 2,670 & 33 & & none & 3,060 & 1,580 & 2,330 & 38 \\
\hline & $\mathrm{bcc}$ & 2,000 & 160 & 390 & 29 & & $\mathrm{bcc}$ & 2,120 & 1,100 & 2,770 & & & no & 4,160 & 2,080 & 1,980 & 36 \\
\hline & none & 3,120 & 40 & 060 & 25 & & & 3,000 & 20 & 2,350 & & & & 3,960 & 40 & 1,800 & 29 \\
\hline & none & 3,660 & 840 & 1,970 & 36 & & $\mathrm{bcc}$ & 1,880 & 1,000 & 2,120 & 20 & & bcc & 2,240 & 1,600 & 2,290 & 24 \\
\hline & one & 2,560 & 740 & 2,420 & 1 & & $\mathrm{cc}$ & , 120 & 880 & 60 & & & $\mathrm{cc}$ & 960 & 1,540 &, 06 & 31 \\
\hline & none & 2,600 & 560 & 130 & 28 & & $\mathrm{bcc}$ & 2,120 &, 760 & 2,500 & & & $\mathrm{cc}$ &, 880 & 2,080 & 0 & 34 \\
\hline & one & 2,760 & 060 & 400 & $t_{1}$ & & bcc & 1,300 & ,520 & 2,360 & & & $\mathrm{cc}$ & 280 & 1,42 &, 0 & 27 \\
\hline & one & 2,600 & 660 & 080 & 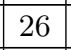 & & bcc & 1,660 & 1,700 & 2,450 & & & $\mathrm{bcc}$ & 1,460 & 1,840 & 2,06 & 30 \\
\hline & ne & 4,060 & 320 & 500 & 34 & & one & 2,600 & 1,840 & 2,210 & 50 & & ione & 3,260 & 1,960 &, 30 & 34 \\
\hline & none & 3,180 & 820 & 2,640 & 32 & & none & 2,260 & 2,340 & 2,180 & 41 & & bcc & 1,260 & 1,580 & 2,150 & 28 \\
\hline & bcc & 1,800 & 2,000 & 2,160 & 26 & & $\mathrm{bcc}$ & 1,660 & 1,880 & 2,590 & 23 & & bcc & 2,040 & 2,060 & 2,130 & 31 \\
\hline & bcc & 1,720 & 1,500 & 1,900 & 31 & & $\mathrm{bcc}$ & 1,360 & 1,680 & 2,210 & 20 & & bcc & 1,820 & 1,840 & 1,890 & 36 \\
\hline & none & 2,620 & 1,600 & 2,300 & 50 & & none & 3,480 & 1,940 & 1,880 & 31 & & $\mathrm{bcc}$ & 2,120 & 2,460 & $2,390 \mid$ & 32 \\
\hline & none & 3,760 & 1,540 & 1,850 & 32 & & none & 2,760 & 1,480 & 2,000 & 30 & & none & 3,860 & 1,740 & $2,490]$ & 34 \\
\hline & fcc & 1,880 & 360 & 2,540 & 30 & & none & 3,140 & 1,760 & 1,980 & & & fcc & 440 & 1,500 & 2,0 & 32 \\
\hline & one & 3,400 & 620 & 2,310 & 32 & & fcc & 1,660 & 160 & 2,190 & & & cc & 1,540 & 1,220 & 2,360 & 29 \\
\hline & none & 2,640 & 360 & 030 & (n) & & none & 1,960 & 280 & 1,770 & 50 & & bcc & 1,560 & 1,240 & 2,12 & 20 \\
\hline & none & 500 & 600 & 100 & & & ne & 2,520 & 0 & 1,860 & 2 & & none & 2,280 & 1,200 & 2,0 & 16 \\
\hline & $\tau$ & 880 & 260 & 560 & 17 & & & 5,000 & 00 & 1,750 & 4 & & no & 2,760 & 1,480 & 1,6 & 50 \\
\hline & ne & 3,160 & 400 & 030 & 25 & & e & 4,380 &, 720 & 2,030 & 24 & & none & 3,240 & 1,640 & 1,76 & 27 \\
\hline & one & 3,020 & 520 & 900 & 18 & & none & 2,600 & 1,340 & 2,520 & 21 & & nor & 2,680 & 1,900 & 1,720 & 42 \\
\hline & $\mathrm{bcc}$ & 1,700 & 240 & 1,780 & 50 & & none & 2,060 & 1,180 & 1,660 & 49 & & none & 4,040 & 1,680 &, 130 & 28 \\
\hline & none & 1,900 & 320 & 1,890 & 29 & & $\mathrm{bcc}$ & 1,440 & 800 & 2,080 & 21 & & bcc & 1,780 & 1,380 & 2,700 & 22 \\
\hline & none & 2,600 & 2,020 & 1,800 & 47 & & none & 4,060 & 1,900 & 1,870 & 28 & & none & 4,100 & 1,960 & 1,980 & 18 \\
\hline $\mathrm{aW}$ & none & 3,580 & 1,840 & 2,650 & 21 & $\mathrm{aZr}$ & none & 3,800 & 2,340 & 1,780 & 44 & & none & 2,640 & 1,300 & 1,770 & 23 \\
\hline $\mathrm{iW}$ & none & 3,380 & 1,540 & 2,320 & 25 & TiZr & none & 3,060 & 1,920 & 1,600 & 47 & $\mathrm{JW}$ & bcc & 2,240 & 1,180 & 2,370 & 17 \\
\hline $\mathrm{Zr}$ & ne & 3,640 & 060 & 2,360 & 40 & $\mathrm{DNi}$ & none & 2,200 & 280 & 2,040 & 50 & $\operatorname{Re}$ & bcc & 2,360 & 1,180 & 2,510 & 13 \\
\hline $\mathrm{Ta}$ & bcc & 2,500 & 1,100 & 2,550 & 10 & & bcc & 1,860 & 0 & 2,200 & 14 & & bcc & 2,220 & 1,000 & 2,300 & 6 \\
\hline & one & 2,880 & 920 & 2,630 & 6 & & none & 2,940 & 980 & 2,090 & 2 & & $\mathrm{cc}$ & 1,580 & 960 & 2,18 & 50 \\
\hline & one & 3,040 & 1,660 & 30 & 17 & & b & 1,960 & 420 & 2 , & & & $\mathrm{bcc}$ & 1,140 & 940 & 2,47 & 11 \\
\hline & bcc & 340 & 740 & 2,760 & 8 & & none & 2,500 & 1,500 & 2,160 & 29 & & bcc & 1,600 & 1,340 & 2,32 & 15 \\
\hline & $\mathrm{bcc}$ & 040 & 1,140 & 2,400 & 6 & & $\mathrm{bcc}$ & 2,620 & 1,040 & 2,790 & & & none & 3,220 & 2,180 & 2,18 & 37 \\
\hline & bcc & 580 & 920 & 2,110 & 14 & iW & bcc & 1,820 & 960 & 2,370 & 15 & $\mathrm{iZr}$ & none & 2,140 & 1,520 & 1,910 & 28 \\
\hline & bcc & 1,500 & 660 & 2,530 & - & & none & 3,640 & 3,100 & 1,970 & 25 & $\mathrm{VZr}$ & none & 4,100 & 2,780 & 2,430 & 22 \\
\hline $\mathrm{CrNbNiV}$ & none & 2,240 & 1,380 & 1,810 & 47 & CrNbReTa & none & 3,280 & 1,600 & 2,560 & 19 & CrNbReTi & bcc & 2,200 & 1,520 & 2,220 & 24 \\
\hline
\end{tabular}




\begin{tabular}{|c|c|c|c|c|c|c|c|c|c|c|c|c|c|c|c|c|c|}
\hline & S. & $T_{\mathrm{c}}$ & $\tilde{T}_{\mathrm{c}}$ & $\bar{T}_{\mathrm{m}}$ & $\mathrm{CV}$ & & S.S. & $T_{\mathrm{c}}$ & $\tilde{T}_{\mathrm{c}}$ & $\bar{T}_{\mathrm{m}}$ & 10 & & & $T_{\mathrm{c}}$ & $I_{\mathrm{C}}$ & $\bar{T}_{\mathrm{m}}$ & CV \\
\hline & one & 2,380 & 1,340 & 2,350 & 16 & & $\mathrm{bcc}$ & 2,160 & 1,240 & 640 & 14 & ReZr & one & 680 & 020 & 2,120 & 30 \\
\hline & $\mathrm{ccc}$ & 340 & 880 & 250 & 13 & & $\mathrm{cc}$ & 280 & 1,020 & 2,300 & & & 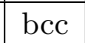 & 2,340 & 840 & 680 & 7 \\
\hline & one & 2,380 & 240 & 2,160 & 19 & & $\mathrm{cc}$ & 820 & 1,100 & 1,990 & 13 & & $\mathrm{cc}$ & 660 & 1,040 & 250 & 14 \\
\hline $\mathrm{Cr}$ & none & 3,900 & 2,220 & 1,880 & 24 & & $\mathrm{bcc}$ & 1,980 & 880 & 2,380 & & & none & 4,400 & 3,360 & ,910 & 23 \\
\hline CrNbWZr & none & 4,600 & 3,240 & 2,360 & 20 & sPd & fcc & 1,600 & 1,560 & 2,250 & 34 & $\mathrm{sPt}$ & none & 3,040 & 1,540 &, 300 & 33 \\
\hline CrNiPdPt & none & 3,960 & 1,460 & 1,710 & 31 & CrNiPdRh & none & 3,080 & 1,340 & 1,720 & 32 & $\mathrm{dRu}$ & none & 2,560 & 1,440 & 2,070 & 31 \\
\hline CrNiPtRh & one & 3,160 & 1,300 & 1,860 & 30 & CrNiPtRu & none & 2,600 & 240 & 2,120 & 31 & $\mathrm{ReV}$ & $\mathrm{bcc}$ & 1,700 & 1,200 & 2,050 & 50 \\
\hline $\mathrm{CrOs}$ & one & 3,220 & 900 & 2,330 & 36 & $\mathrm{CrO}$ & none & 2,540 & 1,600 & 2,380 & 34 & $\mathrm{dRu}$ & none & 2,980 & 1,740 & 2,460 & 34 \\
\hline $\mathrm{CrO}$ & fcc & 1,340 & 420 & 2,430 & 31 & tRu & one & 2,540 & 1,480 & 2,520 & 31 & & none & 2,280 & 1,420 & 890 & 33 \\
\hline $\mathrm{CrP}$ & ne & 2,980 & 560 & 150 & 35 & & ne & 2,720 & 240 & 2,190 & 33 & & fcc & 1,800 & 960 & 250 & 30 \\
\hline & ne & 2,880 & 300 & 370 & 26 & & none & 960 & 580 & 2,480 & 18 & & ione & 2,920 & 1,500 & 2,83 & 17 \\
\hline & one & 3,600 & 280 & 240 & 32 & & bcc & 1,680 & 260 & 2,190 & ת & & & 2,440 & 1,020 & 42 & 24 \\
\hline & none & 2,280 & 820 & 1,980 & 34 & & $\mathrm{cc}$ & 1,180 & 980 & 2,600 & 12 & & none & 3,260 & 2,020 & 2,060 & 33 \\
\hline & none & 2,580 & 800 & 2,480 & 29 & & $\mathrm{cc}$ & 1,780 & 1,380 & 2,120 & 14 & & $\mathrm{bcc}$ & 1,320 & 1,200 & 2,440 & 14 \\
\hline & none & 3,400 & 340 & 1,990 & 24 & & $\mathrm{cc}$ & 1,460 & 960 & 2,550 & & & none & 4,080 & 3,220 & 2,000 & 24 \\
\hline & none & 4,180 & 3,040 & 2,510 & 21 & & $\mathrm{cc}$ & 1,220 & 780 & 2,170 & 13 & & none & 3,000 & 1,600 & 1,770 & 28 \\
\hline $\mathrm{Cr}$ & none & 2,740 & 1,760 & 2,160 & 26 & & none & 4,900 & 1,740 & 2,240 & 26 & & none & 2,140 & 1,300 & 1,920 & 47 \\
\hline $\mathrm{nNb}$ & none & 3,400 & 1,860 & 1,670 & 50 & $\mathrm{nTa}$ & none & 4,620 & 2,540 & 1,600 & 50 & $\mathrm{nTi}$ & none & 3,720 & 2,080 & 1,470 & 50 \\
\hline $\mathrm{CuF}$ & one & 4,800 & 2,000 & 1,730 & 45 & IoTi & none & 3,160 & 1,900 & 1,710 & 50 & & none & 3,900 & 1,560 & 1,940 & 45 \\
\hline $\mathrm{Ta}$ & ne & 3,340 & 1,740 & 1,990 & 50 & & & 3,740 & 740 & 1,760 & 49 & & none & 3,020 & 1,680 & 1,970 & 44 \\
\hline & e & 1,880 & 20 & 1,590 & 38 & & & 2,600 & 060 & 1,740 & & & fec & 740 & 1,860 & 2,080 & 41 \\
\hline $\mathrm{Cu}$ & fcc & 1,160 & 1,740 & 2,110 & 41 & & none & 2,360 & 1,020 & 1,810 & 44 & & none & 4,240 & 1,560 & 1,890 & 33 \\
\hline & one & 3,380 & 300 & 930 & 43 & & none & 3,420 & 80 & 1,750 & 41 & & non & 4,600 & 860 &, 1 & 48 \\
\hline & one & 3,200 & 160 & 780 & 1 & & none & 2,860 & ,000 & 1,920 & 1 & & fcc & 480 & 2,020 &, 30 & 37 \\
\hline & one & 5,000 & 000 &, 350 & & & ione & 4,060 & 120 & 1,890 & 20 & & 100 & 5,000 & 5 & 3 & 49 \\
\hline & ne & 3,760 & 200 & 920 & 0 & & one & 5,000 & 5,000 & 10 & 0 & & none & 5,000 & 5,000 &, 38 & 38 \\
\hline & ne & 2,360 & 00 & 070 & 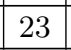 & & 11 & 5,000 & 5,000 & 2,170 & 31 & & none & 4,680 & 2,240 & 1,72 & 50 \\
\hline & ne & 4,800 & 900 & 810 & 48 & & none & 4,800 & 2,180 & 2,360 & 50 & $\mathrm{Ta}$ & non & 4,360 & 2,140 & 1,800 & 50 \\
\hline & none & 3,980 & 2,020 & 1,850 & 50 & & none & 4,540 & 2,240 & 1,780 & 47 & & none & 5,000 & 5,000 & 2,460 & 50 \\
\hline & none & 3,520 & 2,020 & 1,650 & 50 & & none & 5,000 & 5,000 & 2,180 & 50 & bTa & none & 4,400 & 1,520 & 2,250 & 49 \\
\hline & none & 3,700 & 1,460 & 2,190 & 47 & $\mathrm{bW}$ & none & 5,000 & 5,000 & 2,480 & 50 & $\mathrm{TTi}$ & none & 3,160 & 1,740 & 1,950 & 50 \\
\hline & none & 5,000 & 000 & 2,480 & 47 & & none & 2,900 & 1,520 & 1,920 & 46 & & none & 5,000 & 5,000 & 2,400 & 50 \\
\hline & ne & 5,000 & 000 & 1,990 & 50 & & fcc & 900 & 040 & 2,040 & 50 & & none & 5,000 & 5,000 & 2,10 & 50 \\
\hline $\mathrm{Ti}$ & none & 2,300 & 280 & 2,030 & 50 & & none & 2,300 & 1,520 & 2,190 & 45 & & non & 5,000 & 5,000 & 2,530 & 48 \\
\hline & bcc & 1,860 & 480 & 960 & - & & none & 4,360 & 1,600 & 2,410 & sc & & s & 1,220 & 1,82 & 2,06 & 34 \\
\hline & $\mathrm{fc}$ & 920 & 1,640 & 110 & & & fcc & 1,240 & 680 & 1,640 & 11 & & $\mathrm{cc}$ & 1,880 & 1,840 & 2,0 & 36 \\
\hline & ne & 2,360 & 40 & 640 & & & e & 3,700 & 1,480 & 370 & 2 & & $\mathrm{cc}$ & 1,640 & 1,54 & 2,1 & 36 \\
\hline & 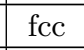 & 640 & 840 & 760 & 13 & & fcc & 1,160 & 180 & 1,930 & 22 & & tcc & 360 & 1,680 & 2,1 & 35 \\
\hline & ne & 5,000 & 000 & 2,490 & 50 & & none & 5,000 & 5,000 & 2,190 & 41 & & none & 5,000 & 5,000 & 2,27 & 50 \\
\hline & fcc & 980 & 2,360 & 2,540 & 42 & & fcc & 1,880 & 1,640 & 2,240 & 37 & & fcc & 1,320 & 2,100 & 2,330 & 35 \\
\hline & fcc & 900 & 1,660 & 2,170 & \begin{tabular}{|l|}
39 \\
\end{tabular} & & none & 1,980 & 780 & 1,790 & \begin{tabular}{|l|}
14 \\
\end{tabular} & & fcc & 280 & 1,160 & 1,950 & 24 \\
\hline & fcc & 860 & 2,100 & 2,220 & 34 & $\mathrm{Ru}$ & none & 5,000 & 5,000 & 2,300 & 38 & $\mathrm{Ru}$ & $\mathrm{fcc}$ & 420 & 1,500 & 2,000 & 32 \\
\hline $\mathrm{Rh}$ & $\mathrm{fcc}$ & 780 & 1,680 & 2,270 & 35 & $\mathrm{Ru}$ & fcc & 880 & 880 & 2,360 & 36 & $\mathrm{hRu}$ & fcc & 1,560 & 1,140 & 2,060 & 36 \\
\hline $\mathrm{Cr}$ & none & 2,360 & 780 & 1,930 & 44 & $\mathrm{iW}$ & none & 3,760 & 1,860 & 2,210 & \begin{tabular}{|l|}
50 \\
\end{tabular} & $\overline{V W}$ & none & 4,520 & 1,620 & 2,420 & 50 \\
\hline & none & 3,580 & 800 & 120 & 46 & & $\mathrm{nc}$ & 4,200 & 1,800 & 2,300 & 5 & & fcc & 1,900 & 1,040 & 2,01 & 50 \\
\hline & fcc & 880 & 520 & 410 & 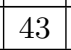 & & $\mathrm{n}$ & 2,580 & 0 & 2,440 & 4 & & fcc & 640 & 1,420 & 2,230 & 43 \\
\hline & none & 2,360 & 540 & 980 & 3 & & bcc & 2,300 & 220 & 2,420 & 35 & & ione & 3,140 & 1,620 & 2,030 & 34 \\
\hline & one & 3,280 & 680 & 00 & 32 & & none & 1,960 & 0 & 1,920 & 20 & & none & 2,700 & 1,560 & 2,47 & 42 \\
\hline & none & 2,960 & 480 & 380 & 39 & & nor & 1,360 & 1,86 & 1,980 & 31 & Ti & none & 4,500 & 2,240 & 1,73 & 41 \\
\hline & none & 2,720 & 640 & 830 & 26 & $\mathrm{~W}$ & none & 2,460 & 1,420 & 2,440 & 39 & $\mathrm{Ta}$ & none & 4,000 & 1,600 & 2,51 & 38 \\
\hline eTi & bcc & 040 & 280 & 2,180 & 40 & & bcc & 1,740 & 920 & 2,240 & 34 & & bcc & 1,740 & 1,520 & 2,620 & 44 \\
\hline & none & 5,000 & 5,000 & 1,820 & 47 & & none & 3,820 & 1,820 & 1,890 & 29 & $\mathrm{aW}$ & none & 2,760 & 1,520 & 2,570 & 37 \\
\hline FeMnTiV & none & 4,280 & 2,060 & 1,690 & 32 & FeMnTiW & none & 4,020 & 1,880 & 2,240 & 39 & FeMnVW & $\mathrm{bcc}$ & 2,120 & 1,260 & 2,290 & 30 \\
\hline
\end{tabular}









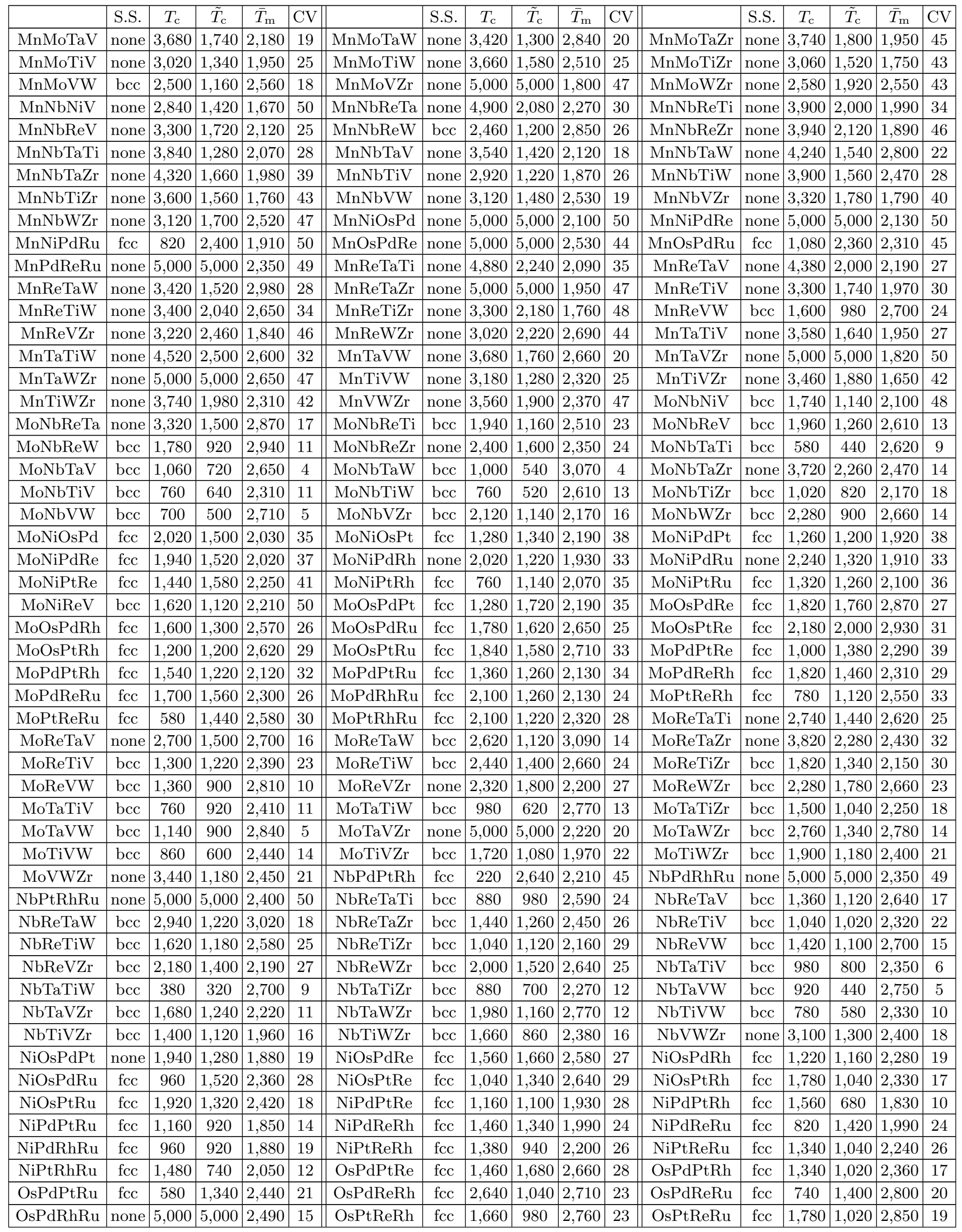




\begin{tabular}{|c|c|c|c|c|c||c|c|c|c|c|c||c|c|c|c|c|c|}
\hline & S.S. & $T_{\mathrm{c}}$ & $\tilde{T}_{\mathrm{c}}$ & $\bar{T}_{\mathrm{m}}$ & CV & & S.S. & $T_{\mathrm{c}}$ & $\tilde{T}_{\mathrm{c}}$ & $\bar{T}_{\mathrm{m}}$ & CV & & S.S. & $T_{\mathrm{c}}$ & $\tilde{T}_{\mathrm{c}}$ & $\bar{T}_{\mathrm{m}}$ & CV \\
\hline OsPtRhRu & fcc & 1,500 & 860 & 2,550 & 12 & PdPtReRh & fcc & 1,680 & 1,000 & 2,240 & 26 & PdPtReRu & fcc & 1,560 & 1,320 & 2,270 & 25 \\
\hline PdPtRhRu & fcc & 840 & 800 & 2,090 & 12 & PdReRhRu & none & 2,600 & 900 & 2,310 & 20 & PtReRhRu & fcc & 1,680 & 840 & 2,570 & 21 \\
\hline ReTaTiV & bcc & 1,080 & 1,160 & 2,440 & 24 & ReTaTiW & bcc & 2,080 & 1,300 & 2,760 & 26 & ReTaTiZr & bcc & 1,460 & 1,460 & 2,260 & 30 \\
\hline ReTaVW & bcc & 2,320 & 1,280 & 2,870 & 18 & ReTaVZr & bcc & 2,240 & 1,640 & 2,270 & 29 & ReTaWZr & none & 2,940 & 1,940 & 2,780 & 27 \\
\hline ReTiVW & bcc & 1,420 & 1,240 & 2,470 & 24 & ReTiVZr & none & 2,040 & 1,440 & 2,030 & 30 & ReTiWZr & bcc & 1,820 & 1,800 & 2,420 & 31 \\
\hline ReVWZr & bcc & 2,460 & 1,700 & 2,490 & 29 & TaTiVW & bcc & 720 & 800 & 2,490 & 10 & TaTiVZr & bcc & 1,220 & 1,440 & 2,040 & 16 \\
\hline TaTiWZr & bcc & 2,040 & 1,080 & 2,530 & 16 & TaVWZr & none & 3,520 & 1,560 & 2,530 & 18 & TiVWZr & none & 2,540 & 1,260 & 2,190 & 21 \\
\hline
\end{tabular}

\section{Quinary alloys at equi-composition}

TABLE VI: LTVC results for quinary alloys. S.S. type of single solid solution phase ("none" = not found); $T_{\mathrm{c}}(\mathrm{K})$ transition temperature, as estimated by the method; $\tilde{T}_{\mathrm{c}}(\mathrm{K})$ approximated transition temperature, estimated according to Eq. (8); CV $(\mathrm{meV})$ cluster expansion cross validation score; $\bar{T}_{\mathrm{m}}(\mathrm{K})$ melting temperature (solidus), calculated as the average of the melting temperatures at equi-composition of the 10 binary alloys associated with the quinary alloy; A solid solution is predicted if $T_{\mathrm{c}}<\bar{T}_{\mathrm{m}}$.

\begin{tabular}{|c|c|c|c|c|c|c|c|c|c|c|c|}
\hline & S. & $T_{\mathrm{c}}$ & $\tilde{T}_{\mathrm{c}}$ & $\bar{T}_{\mathrm{m}}$ & $\mathrm{CV}$ & & S.S. & $T_{\mathrm{c}}$ & $\tilde{T}_{\mathrm{c}}$ & $\bar{T}_{\mathrm{m}}$ & $\mathrm{CV}$ \\
\hline & $\mathrm{bcc}$ & .650 & $\overline{000}$ & 2,150 & 44 & $\overline{\mathrm{Ti}}$ & $\overline{c c}$ & 850 & $\overline{950}$ & & 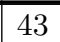 \\
\hline & c & & 50 & & 19 & & & & D- & & \\
\hline & ${ }^{2}$ & & & & 21 & & & & & & \\
\hline & $c c$ & & & & & & & & & & \\
\hline & ne & 300 & 50 & 99 & 22 & & & 00 & 1 & 02 & \\
\hline & bcc & 050 & 00 & 200 & 26 & & none & 200 & ,100 & 2,06 & \\
\hline & $\mathrm{bcc}$ & 750 & 00 & 320 & 24 & & bcc & 1,750 & 35 & ,99 & \\
\hline & $\mathrm{bcc}$ & 300 & 50 & 390 & 22 & & one & 3,000 & 45 & 00 & \\
\hline & $\mathrm{bcc}$ & 750 & 00 & 380 & 27 & & $\mathrm{cc}$ & 450 & D. &, 24 & 11 \\
\hline & none & 50 & 00 & 50 & 20 & & $\mathrm{bcc}$ & 50 & & 2,590 & 8 \\
\hline & one & 00 & 00 & 220 & 31 & & 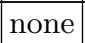 & 2 & 000 & 2 & \\
\hline & $\mathrm{bcc}$ & 50 & & 270 & 22 & & $c$ & & & 2,00 & \\
\hline & bcc & 200 & 0 & & & & on & 3 & & 2,00 & \\
\hline & bcc & 700 & 50 & 370 & - & & & & U & 2 & \\
\hline & bcc & 200 & & 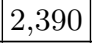 & 22 & & CC & 700 & 45 & 01 & 27 \\
\hline & $\mathrm{bcc}$ & 800 & 550 & 30 & $-x$ & & ion & 2,750 & 35 & 2 & \\
\hline & $\mathrm{bcc}$ & 250 & 200 & 460 & 25 & & $c c$ & 650 & 00 & 2,15 & 24 \\
\hline & ne & 2,850 & 550 & 870 & 31 & & $\mathrm{cc}$ & 1,750 & 1,150 & 2,200 & - \\
\hline & none & 3,050 & 100 & 30 & 29 & & , & 50 & 900 & 90 & 2 \\
\hline & $\mathrm{bcc}$ & 350 & 900 & 0 & 8 & & none & 2,750 & 900 & 40 & \\
\hline & none & 2,900 & ,400 & 290 & 22 & & $\mathrm{bcc}$ & 900 & 800 & 180 & 12 \\
\hline & bc & & & 10 & 1 & & & 00 & 15 & 2,0 & \\
\hline & & & & 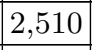 & & & 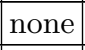 & 050 & 25( & 2 & \\
\hline & ne & 50 & 400 & 430 & & & bcc & 1,70 & 800 & 2,27 & 2 \\
\hline & cc & 600 & & 10 & $1:$ & & none & 2,150 & 1,3 & 2,13 & 1 \\
\hline & ¿ct & 00 & 850 & & 2 & & none & 3,450 & 1,4 & 2,1 & 0 \\
\hline & 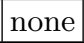 & 100 & 450 & . & 26 & & $\mathrm{bc}$ & 500 & & {$[, 02$} & 13 \\
\hline & none & 2,800 & 200 & 50 & 24 & & none & 2,500 &, 05 & 2,250 & 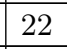 \\
\hline & none & 900 & 1,200 & 320 & 21 & & $\mathrm{bcc}$ & 800 & 00 & 2,200 & 11 \\
\hline & bcc & 550 & 650 & 2,460 & 12 & & bcc & 1,800 & 1,150 & 2,110 & 19 \\
\hline & bcc & 150 & 800 & 530 & 7 & & none & 2,850 & 1,30 & 2,120 & 22 \\
\hline & & 650 & 350 & 500 & 1 & & $\mathrm{bcc}$ & 1,750 & 700 & 2,220 & 12 \\
\hline & & 0 & &, 900 & 22 & & & 225 & 1000 & 2,200 & \\
\hline
\end{tabular}




\begin{tabular}{|c|c|c|c|c|c|c|c|c|c|c|c|}
\hline & S. & $T_{\mathrm{c}}$ & $\tilde{T}_{\mathrm{c}}$ & $\bar{T}_{\mathrm{m}}$ & $\mathrm{CV}$ & & S.S. & $T_{\mathrm{c}}$ & $\tilde{T}_{\mathrm{c}}$ & $\bar{T}_{\mathrm{m}}$ & $\omega_{0}$ \\
\hline $\mathrm{Zr}$ & none & 000 & 450 & 2,260 & 21 & CrTaTiVW & $\mathrm{ccc}$ & 400 & 700 & 2,360 & \\
\hline & & 50 & & & 22 & & ne & 50 & 0 & 330 & 20 \\
\hline & & & & & 24 & & & & & & \\
\hline & & & & & 17 & & & & & & \\
\hline & & & & & 14 & & & & & & 17 \\
\hline & & & & 30 & 20 & & & 21 & & jo & 19 \\
\hline & $\mathrm{cc}$ & 400 & & 190 & 22 & & cc & , 60 & 50 & , זלכ, & \\
\hline & $\mathrm{cc}$ & 450 & & 170 & 22 & & $\mathrm{cc}$ & 400 & 50 &, 57 & \\
\hline & $\mathrm{cc}$ & 450 & 550 & 280 & 20 & & cc & 350 & 50 & 60 & \\
\hline & $\mathrm{bcc}$ & 00 & 950 & 240 & 22 & & $\mathrm{cc}$ & 650 & 00 &, 62 & 7 \\
\hline & $\mathrm{bcc}$ & 600 & 950 & 00 & 22 & & $\mathrm{cc}$ & 850 & 50 &, 630 & 9 \\
\hline & bcc & 550 & 80 & 330 & 22 & & $\mathrm{cc}$ & 800 & 05 & 2,000 & \\
\hline & $\mathrm{ccc}$ & 350 & & 50 & 2 & & $\mathrm{cc}$ & 150 & 1 & 2,370 & 5 \\
\hline & $\mathrm{cc}$ & & & 280 & 16 & & & 25 & & 2,596 & 5 \\
\hline & & & & 30 & 1 & & & & & 2,600 & \\
\hline & $\mathrm{bc}$ & 6 & & 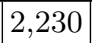 & 16 & & & & & 2,6 & 16 \\
\hline & $\mathrm{bc}$ & 4 & 75 & 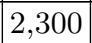 & 16 & & & 750 & & 2,02 & 21 \\
\hline & $\mathrm{bc}$ & 600 & 750 & 30 & 20 & & bcc & 200 & 950 & 2,37 & 21 \\
\hline & bcc & 600 & 80 & 390 & 17 & & none & 2,750 & . & 2,07 & 23 \\
\hline & 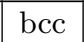 & 950 & 900 & 460 & 20 & & $\mathrm{cc}$ & 2,250 & 0 & 2,44 & 1 \\
\hline & none & 2,300 & 050 & 190 & 23 & & $\mathrm{cc}$ & 2,750 & $1-1$ & 000 & 4 \\
\hline & bcc & 0 & 450 & 0 & 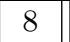 & & $\mathrm{cc}$ & 50 & 450 & 2,750 & 10 \\
\hline & $\mathrm{cc}$ & 350 & 15 & & 13 & & $\mathrm{cc}$ & 1,000 & $\overline{500}$ & 800 & \\
\hline & ne & 3,550 & 1,100 & 350 & 1 & & cc & 2,650 & 50 &, 750 & 11 \\
\hline & $\mathrm{cc}$ & & 45 & 480 & & & DC & 50 & 60 &, 12 & 17 \\
\hline & & & & 50 & 1 & & e & 3 & 00 & 48 & 16 \\
\hline & & & & 0 & 1 & & $b c$ & 1,750 & & 2,180 & 17 \\
\hline & $\mathrm{CC}$ & & & 10 & 17 & & none & 3,400 & 1,0 & 2,56 & 7 \\
\hline & bne & 2,55 & 9 & 90 & 20 & & $\mathrm{bcc}$ & 950 & & 2,47 & 9 \\
\hline & bcc & & 40 & $0<0$ & & & Or & 3,10 & 1,1 & 2,17 & \\
\hline & $\mathrm{bcc}$ & 000 & 00 & 530 & 13 & & nor & 2,850 & 85 & 2,530 & 14 \\
\hline NbTiVWZ1 & $\mathrm{bcc}$ & 2,050 & 750 & 250 & 17 & WZr & non & 2,40 & 800 & 2,350 & 17 \\
\hline
\end{tabular}

[1] J.-W. Yeh, S.-K. Chen, S.-J. Lin, J.-Y. Gan, T.-S. Chin, T.-T. Shun, C.-H. Tsau, and S.-Y. Chang, Nanostructured High-Entropy Alloys with Multiple Principle Elements: Novel Alloy Design Concepts and Outcomes, Adv. Eng. Mater. 6, 299-303 (2004).

[2] B. Cantor, I. T. H. Chang, P. Knight, and A. J. B. Vincent, Microstructural development in equiatomic multicomponent alloys, Mater. Sci. Eng. A 375, 213-218 (2004).

[3] O. N. Senkov, J. D. Miller, D. B. Miracle, and C. Woodward, Accelerated exploration of multi-principal element alloys with solid solution phases, Nature Commun. 6, 6529 (2015).

[4] S. Gorsse, D. B. Miracle, and O. N. Senkov, Mapping the world of complex concentrated alloys, Acta Mater. 135,
177-187 (2017)

[5] X. Lim, Mixed-up metals make for stronger, tougher, stretchier alloys, Naturere 533, 306-307 (2016).

[6] B. Gludovatz, A. Hohenwarter, D. Catoor, E. H. Chang, E. P. George, and R. O. Ritchie, A fracture-resistant highentropy alloy for cryogenic applications, Science $\mathbf{3 4 5}$, 1153-1158 (2014).

[7] F. von Rohr, M. J. Winiarski, J. Tao, T. Klimczuk, and R. J. Cava, Effect of electron count and chemical complexity in the Ta-Nb-Hf-Zr-Ti high-entropy alloy superconductor, Proc. Nature. Acad. Sci. 113, E7144-E7150 (2016).

[8] Z. Li, K. G. Pradeep, Y. Deng, D. Raabe, and C. C. Tasan, Metastable high-entropy dual-phase alloys overcome the strength-ductility trade-off, Naturere 534, 227- 
230 (2016).

[9] J.-W. Yeh, A.-C. Yeh, and S.-Y. Chang, Potential Applications and Prospects, in High-Entropy Alloys: Fundamentals and Applications, edited by M. C. Gao, J.-W. Yeh, P. K. Liaw, and Y. Zhang (Springer, Cham, Switzerland, 2015), chap. 15.

[10] C. M. Rost, E. Sachet, T. Borman, A. Moballegh, E. C. Dickey, D. Hou, J. L. Jones, S. Curtarolo, and J.-P. Maria, Entropy-stabilized oxides, Nature Commun. 6, 8485 (2015).

[11] S. Guo and C. T. Liu, Phase stability in high entropy alloys: Formation of solid-solution phase or amorphous phase, Prog. Nature Sci. Mater. Int. 21, 433-446 (2011).

[12] Y. Zhang, S. Guo, C. T. Liu, and X. Yang, Phase Formation Rules, in High-Entropy Alloys: Fundamentals and Applications, edited by M. C. Gao, J.-W. Yeh, P. K. Liaw, and Y. Zhang (Springer, Cham, Switzerland, 2015), chap. 2.

[13] M. C. Gao, Design of High-Entropy Alloys, in HighEntropy Alloys: Fundamentals and Applications, edited by M. C. Gao, J.-W. Yeh, P. K. Liaw, and Y. Zhang (Springer, Cham, Switzerland, 2015), chap. 11.

[14] Y. Zhang, Y. J. Zhou, J. P. Lin, G. L. Chen, and P. K. Liaw, Solid-Solution Phase Formation Rules for Multicomponent Alloys, Adv. Eng. Mater. 10, 534-538 (2008).

[15] M. G. Poletti and L. Battezatti, Electronic and thermodynamic criteria for the occurrence of high entropy alloys in metallic systems, Acta Mater. 75, 297-306 (2014).

[16] Y. F. Ye, C. T. Liu, and Y. Yang, A geometric model for intrinsic residual strain and phase stability in high entropy alloys, Acta Mater. 94, 152-161 (2015).

[17] Y. F. Ye, Q. Wang, J. Lu, C. T. Liu, and Y. Yang, Design of high entropy alloys: A single parameter thermodynamic rule, Scr. Mater. 104, 53-55 (2015).

[18] S. Guo, C. Ng, Z. Wang, and C. T. Liu, Solid solutioning in equiatomic alloys: Limit set by topological instability, J. Alloys Compd. 583, 410-413 (2014).

[19] S. Guo, C. Ng, J. Lu, and C. T. Liu, Effect of valence electron concentration on stability of fcc or bcc phase in high entropy alloys, J. Appl. Phys. 109, 103505 (2011).

[20] M. C. Troparevsky, J. R. Morris, P. R. C. Kent, A. R. Lupini, and G. M. Stocks, Criteria for Predicting the Formation of Single-Phase High-Entropy Alloys, Phys. Rev. X 5, 011041 (2015).

[21] M. C. Gao and D. E. Alman, Searching for Next SinglePhase High-Entropy Alloy Compositions, Entropy 15, 4504-4519 (2013).

[22] F. Zhang, C. Zhang, S. L. Chen, J. Zhu, W. S. Cao, and U. R. Kattner, An understanding of high entropy alloys from phase diagram calculations, Calphad 45, 1-10 (2014).

[23] M. Widom, Prediction of Structure and Phase Transformations, in High-Entropy Alloys: Fundamentals and Applications, edited by M. C. Gao, J.-W. Yeh, P. K. Liaw, and Y. Zhang (Springer, Cham, Switzerland, 2015), chap. 8.

[24] W. P. Huhn and M. Widom, Prediction of A2 to B2 Phase Transition in the High Entropy Alloy $\mathrm{Mo}-\mathrm{Nb}-\mathrm{Ta}-\mathrm{W}$, JOM 65, 1772-1779 (2013).

[25] M. Widom, Entropy and diffuse scattering: comparison of NbTiVZr and CrMoNbV, Metall. Mater. Trans. A 47, 3306-3311 (2016).

[26] D. de Fontaine, Cluster Approach to Order-Disorder Transformations in Alloys, in Solid State Physics, edited by H. Ehrenreich and D. Turnbull (Wiley, New York, 1994), vol. 47, pp. 33-176.

[27] A. van de Walle and M. Asta, Self-driven lattice-model Monte Carlo simulations of alloy thermodynamic properties and phase diagrams, Modelling Simul. Mater. Sci. Eng. 10, 521 (2002).

[28] S. Curtarolo, G. L. W. Hart, M. Buongiorno Nardelli, N. Mingo, S. Sanvito, and O. Levy, The high-throughput highway to computational materials design, Nature Mater. 12, 191-201 (2013).

[29] S. Curtarolo, W. Setyawan, G. L. W. Hart, M. Jahnátek, R. V. Chepulskii, R. H. Taylor, S. Wang, J. Xue, K. Yang, O. Levy, M. J. Mehl, H. T. Stokes, D. O. Demchenko, and D. Morgan, AFLOW: An automatic framework for highthroughput materials discovery, Comput. Mater. Sci. 58, 218-226 (2012).

[30] W. Setyawan and S. Curtarolo, High-throughput electronic band structure calculations: Challenges and tools, Comput. Mater. Sci. 49, 299-312 (2010).

[31] K. Yang, C. Oses, and S. Curtarolo, Modeling OffStoichiometry Materials with a High-Throughput AbInitio Approach, Chem. Mater. 28, 6484-6492 (2016).

[32] O. Levy, M. Jahnátek, R. V. Chepulskii, G. L. W. Hart, and S. Curtarolo, Ordered Structures in Rhenium Binary Alloys from First-Principles Calculations, J. Am. Chem. Soc. 133, 158-163 (2011).

[33] O. Levy, G. L. W. Hart, and S. Curtarolo, Structure maps for hcp metals from first-principles calculations, Phys. Rev. B 81, 174106 (2010).

[34] O. Levy, G. L. W. Hart, and S. Curtarolo, Uncovering Compounds by Synergy of Cluster Expansion and HighThroughput Methods, J. Am. Chem. Soc. 132, 4830-4833 (2010).

[35] G. L. W. Hart, S. Curtarolo, T. B. Massalski, and O. Levy, Comprehensive Search for New Phases and Compounds in Binary Alloy Systems Based on PlatinumGroup Metals, Using a Computational First-Principles Approach, Phys. Rev. X 3, 041035 (2013).

[36] M. J. Mehl, D. Hicks, C. Toher, O. Levy, R. M. Hanson, G. L. W. Hart, and S. Curtarolo, The AFLOW Library of Crystallographic Prototypes: Part 1, Comput. Mater. Sci. 136, S1-S828 (2017).

[37] S. Curtarolo, W. Setyawan, S. Wang, J. Xue, K. Yang, R. H. Taylor, L. J. Nelson, G. L. W. Hart, S. Sanvito, M. Buongiorno Nardelli, N. Mingo, and O. Levy, AFLOWLIB.ORG: A distributed materials properties repository from high-throughput ab initio calculations, Comput. Mater. Sci. 58, 227-235 (2012).

[38] R. H. Taylor, F. Rose, C. Toher, O. Levy, K. Yang, M. Buongiorno Nardelli, and S. Curtarolo, $A$ RESTful API for exchanging materials data in the AFLOWLIB.org consortium, Comput. Mater. Sci. 93, 178-192 (2014).

[39] C. E. Calderon, J. J. Plata, C. Toher, C. Oses, O. Levy, M. Fornari, A. Naturen, M. J. Mehl, G. L. W. Hart, M. Buongiorno Nardelli, and S. Curtarolo, The AFLOW standard for high-throughput materials science calculations, Comput. Mater. Sci. 108 Part A, 233-238 (2015).

[40] F. Rose, C. Toher, E. Gossett, C. Oses, M. Buongiorno Nardelli, M. Fornari, and S. Curtarolo, AFLUX: The $L U X$ materials search API for the AFLOW data repositories, Comput. Mater. Sci. 137, 362-370 (2017).

[41] J. M. Sanchez, F. Ducastelle, and D. Gratias, Generalized cluster description of multicomponent systems, Physica A 
128, 334-350 (1984).

[42] A. van de Walle, M. Asta, and G. Ceder, The Alloy Theoretic Automated Toolkit: A User Guide, Calphad 26, 539-553 (2002).

[43] A. Sher, M. van Schilfgaarde, A.-B. Chen, and W. Chen, Quasichemical approximation in binary alloys, Phys. Rev. B 36, 4279 (1987).

[44] M. A. Berding and A. Sher, Electronic quasichemical formalism: Application to arsenic deactivation in silicon, Phys. Rev. B 58, 3853 (1998).

[45] T. B. Massalski, H. Okamoto, P. R. Subramanian, and L. Kacprzak, eds., Binary Alloy Phase Diagrams (American Society for Metals, Materials Park, OH, 1990).

[46] J.-O. Andersson, T. Helander, L. Höglund, P. Shi, and B. Sundman, Thermo-Calc \& DICTRA, computational tools for materials science, Calphad 26, 273-312 (2002).

[47] Thermo-Calc Software, Thermo-Calc Software Database SSOL version 5, http://www.thermocalc.com/productsservices/software/thermo-calc/ (2015).

[48] Z. Wu, H. Bei, F. Otto, G. M. Pharr, and E. P. George, Recovery, recrystallization, grain growth and phase stability of a family of FCC-structured multi-component equiatomic solid solution alloys, Intermetallics 46, 131140 (2014).

[49] M. S. Lucas, G. B. Wilks, L. Mauger, J. A. Muñoz, O. N. Senkov, E. Michel, J. Horwath, S. L. Semiatin, M. B. Stone, D. L. Abernathy, and E. Karapetrova, Absence of long-range chemical ordering in equimolar $\mathrm{FeCoCrNi}$, Appl. Phys. Lett. 100, 251907 (2012).

[50] R. Kozak, A. Sologubenko, and W. Steurer, Single-phase high-entropy alloys - an overview, Zeitschrift für Kristallographie - Crystalline Materials 230, 55 (2015).

[51] N. D. Stepanov, D. G. Shaysultanov, G. A. Salishchev, and M. A. Tikhonovsky, Structure and mechanical properties of a light-weight AlNbTiV high entropy alloy, Mater. Lett. 142, 153-155 (2015).

[52] Y. D. Wu, Y. H. Cai, T. Wang, J. J. Si, J. Zhu, Y. D. Wang, and X. D. Hui, A refractory $H_{25} \mathrm{Nb}_{25} T i_{25} Z r_{25}$ high-entropy alloy with excellent structural stability and tensile properties, Mater. Lett. 130, 277-280 (2014).

[53] O. N. Senkov, G. B. Wilks, D. B. Miracle, C. P. Chuang, and P. K. Liaw, Refractory high-entropy alloys, Intermetallics 18, 1758-1765 (2010).

[54] O. N. Senkov, G. B. Wilks, J. M. Scott, and D. B. Miracle, Mechanical properties of $\mathrm{Nb}_{25} \mathrm{Mo}_{25} \mathrm{Ta}_{25} \mathrm{~W}_{25}$ and $V_{20} \mathrm{Nb}_{20} \mathrm{Mo}_{20} \mathrm{Ta}_{20} \mathrm{~W}_{20}$ refractory high entropy alloys, Intermetallics 19, 698-706 (2011).

[55] X. Yang, Y. Zhang, and P. K. Liaw, Microstructure and Compressive Properties of $\mathrm{NbTiVTaAl}$ High Entropy Alloys, Procedia Engineering 36, 292-298 (2012).

[56] O. N. Senkov, S. V. Senkova, D. B. Miracle, and C. Woodward, Mechanical properties of low-density, refractory multi-principal element alloys of the $\mathrm{Cr}-\mathrm{Nb}-\mathrm{Ti}-\mathrm{V}-\mathrm{Zr}$ system, Mater. Sci. Eng. A 565, 51-62 (2013).

[57] Y. Zhang, X. Yang, and P. K. Liaw, Alloy design and properties optimization of high-entropy alloys, JOM 64, 830-838 (2012).

[58] B. Gorr, M. Azim, H.-J. Christ, T. Mueller,
D. Schliephake, and M. Heilmaier, Phase equilibria, microstructure, and high temperature oxidation resistance of novel refractory high-entropy alloys, J. Alloys Compd. 624, 270-278 (2015).

[59] O. N. Senkov, J. M. Scott, S. V. Senkova, D. B. Miracle, and C. F. Woodward, Microstructure and room temperature properties of a high-entropy TaNbHfZrTi alloy, J. Alloys Compd. 509, 6043-6048 (2011).

[60] H. Bei, Multi-component solid solution alloys having high mixing entropy, USA Patent US A1, 0108502 (2013).

[61] M. C. Gao, B. Zhang, S. Yang, and S. M. Guo, Senary Refractory High-Entropy Alloy HfNbTaTiVZr, Metall. Mater. Trans. A 47, 3333-3345 (2016).

[62] G. L. W. Hart and R. W. Forcade, Algorithm for generating derivative structures, Phys. Rev. B 77, 224115 (2008).

[63] G. L. W. Hart and R. W. Forcade, Generating derivative structures from multilattices: Algorithm and application to hcp alloys, Phys. Rev. B 80, 014120 (2009).

[64] A. van de Walle, Multicomponent multisublattice alloys, nonconfigurational entropy and other additions to the Alloy Theoretic Automated Toolkit, Calphad 33, 266 (2009).

[65] G. Kresse and J. Furthmüller, Efficient iterative schemes for ab initio total-energy calculations using a plane-wave basis set, Phys. Rev. B 54, 11169-11186 (1996).

[66] C. Toher, J. J. Plata, O. Levy, M. de Jong, M. D. Asta, M. Buongiorno Nardelli, and S. Curtarolo, Highthroughput computational screening of thermal conductivity, Debye temperature, and Grüneisen parameter using a quasiharmonic Debye model, Phys. Rev. B 90, 174107 (2014).

[67] C. Toher, C. Oses, J. J. Plata, D. Hicks, F. Rose, O. Levy, M. de Jong, M. D. Asta, M. Fornari, M. Buongiorno Nardelli, and S. Curtarolo, Combining the AFLOW GIBBS and Elastic Libraries to efficiently and robustly screen thermomechanical properties of solids, Phys. Rev. Mater. 1, 015401 (2017).

[68] R. Kikuchi, A Theory of Cooperative Phenomena, Phys. Rev. 81, 988 (1951).

[69] S. Kullback and R. A. Leibler, On Information and Sufficiency, Ann. Math. Stat. 22, 79-86 (1951).

[70] V. Vedral, The role of relative entropy in quantum information theory, Rev. Mod. Phys. 74, 197 (2002).

[71] H. Qian, Relative entropy: Free energy associated with equilibrium fluctuations and nonequilibrium deviations, Phys. Rev. E 63, 042103 (2001).

[72] J. Kristensen, I. Bilionis, and N. Zabaras, Relative entropy as model selection tool in cluster expansion, Phys. Rev. B 87, 174112 (2013).

[73] C. Nyshadham, C. Oses, J. E. Hansen, I. Takeuchi, S. Curtarolo, and G. L. W. Hart, A computational high-throughput search for new ternary superalloys, Acta Mater. 122, 438-447 (2017).

[74] V. Ozolins, C. Wolverton, and A. Zunger, $C u-A u, A g-A u$, $\mathrm{Cu}-\mathrm{Ag}$, and $\mathrm{Ni}-\mathrm{Au}$ intermetallics: First-principles study of temperature-composition phase diagrams and structures, Phys. Rev. B 57, 6427 (1998). 